EFFECT OF THICKNESS-TO-CHORD RATIO ON AERODYNAMICS OF NONSLENDER DELTA WING

A THESIS SUBMITTED TO

THE GRADUATE SCHOOL OF NATURAL AND APPLIED SCIENCES

$\mathrm{OF}$

MIDDLE EAST TECHNICAL UNIVERSITY

BY

MOHAMMAD SHARIFI GHAZIJAHANI

IN PARTIAL FULFILLMENT OF THE REQUIREMENTS

FOR

THE DEGREE OF MASTER OF SCIENCE

IN

MECHANICAL ENGINEERING

DECEMBER 2018 

Approval of the thesis:

\section{EFFECT OF THICKNESS-TO-CHORD RATIO ON AERODYNAMICS OF NON-SLENDER DELTA WING}

submitted by MOHAMMAD SHARIFI GHAZIJAHANI in partial fulfillment of the requirements for the degree of Master of Science in Mechanical Engineering Department, Middle East Technical University by,

Prof. Dr. Halil Kalıpçılar

Dean, Graduate School of Natural and Applied Sciences

Prof. Dr. Mehmet Ali Sahir Arıkan

Head of Department, Mechanical Engineering

Assoc. Prof. Dr. Mehmet Metin Yavuz

Supervisor, Mechanical Engineering Dept., METU

\section{Examining Committee Members:}

Prof. Dr. Mehmet Haluk Aksel

Mechanical Engineering Dept., METU

Assoc. Prof. Dr. Mehmet Metin Yavuz

Mechanical Engineering Dept., METU

Assoc. Prof. Dr. Cüneyt Sert

Mechanical Engineering Dept., METU

Asst. Prof. Dr. Özgür Uğraş Baran

Mechanical Engineering Dept., METU

Assoc. Prof. Dr. Murat Kadri Aktaş

Mechanical Engineering Dept., TOBB ETÜ

Date: 20.12 .2018 
I hereby declare that all information in this document has been obtained and presented in accordance with academic rules and ethical conduct. I also declare that, as required by these rules and conduct, I have fully cited and referenced all material and results that are not original to this work.

Name, Surname: Mohammad Sharifi Ghazijahani

Signature: 


\title{
ABSTRACT \\ EFFECT OF THICKNESS-TO-CHORD RATIO ON AERODYNAMICS OF NON-SLENDER DELTA WING
}

\author{
Sharifi Ghazijahani, Mohammad \\ M.S., Department of Mechanical Engineering \\ Supervisor: Assoc. Prof. Dr. Mehmet Metin Yavuz
}

December 2018, 84 pages

Flow characterization over delta wings have gained attention in recent decades due to their prevailing usage in designs of unmanned air vehicles (UAVs). In literature, only a few studies have reported wing thickness effect on both the aerodynamic performance and detailed flow structure over delta wings.

In the present investigation, the effect of thickness-to-chord $(t / C)$ ratio on aerodynamics of a non-slender delta wing with 45 degree sweep angle is characterized in a low-speed wind tunnel using laser illuminated smoke visualization, surface pressure measurements, particle image velocimetry, and force measurements. The delta wings with $t / C$ ratios varying from $2 \%$ to $15 \%$ are tested at broad ranges of angle of attack and Reynolds number. The results indicate that the effect of $t / C$ ratio on flow structure is quite substantial. Considering the low angles of attack where the wings experience leading edge vortex structure, the strength of the vortex structure increases as the $t / C$ ratio increases. However, low $t / C$ ratio wings have pronounced surface separations at higher angle of attack compared to the high $t / C$ ratio wings. These results are well supported by the force measurements such that high $t / C$ ratio wings induce higher lift coefficients, $\mathrm{C}_{\mathrm{L}}$, at 
low angles of attack, whereas maximum $\mathrm{C}_{\mathrm{L}}$ values are higher and appear at higher angle of attack for low $t / C$ ratio wings. This indicates that low $t / C$ ratio wings are more resistive to the stall condition. Considering the lift-to-drag ratio, $C_{L} / C_{D}$, increase in $t / C$ ratio induces remarkable drop in $\mathrm{C}_{\mathrm{L}} / \mathrm{C}_{\mathrm{D}}$ values.

Keywords: Delta wing, low sweep, aerodynamic forces, wing thickness, thicknessto-chord ratio. 


\title{
KALINLIK-VETER ORANININ DÜŞÜK OK AÇILI DELTA KANAT ÜZERINDEKİ AKIŞ YAPISINA ETKİSI
}

\author{
Sharifi Ghazijahani, Mohammad \\ Yüksek Lisans, Makina Mühendisliği Bölümü \\ Tez Yöneticisi : Doç. Dr. Mehmet Metin Yavuz
}

Aralık 2018, 84 sayfa

Delta kanatlar üzerinde akış karakterizasyonu bu kanatların insansız hava araçları (UAV'ler) tasarımlarında yaygın kullanımı nedeniyle son yıllarda önem kazanan bir konu olmuştur. Literatürde, sadece birkaç çalışma, aerodinamik performans ve delta kanatları üzerindeki ayrıntılı akış yapısı üzerinde kanat kalınlığı etkisini bildirmiştir.

$\mathrm{Bu}$ araştırmada, kalınlık veter oranının $t / C 45$ derece ok açılı delta kanatların aerodinamik yapısı üzerindeki etkisi, düşük hızlı bir rüzgar tünelinde, yüzey basınç ölçümü, dumanla akış görüntüleme, parçacık görüntülemeli hız ölçüm tekniği ve kuvvet ölçümü teknikleri kullanılarak incelenmiştir.Kalınlık-veter oranı \%2 ile \% 15 arasında değişen delta kanatlar, geniş atak açısı ve Reynolds sayısı aralığında incelenmiştir. Sonuçlar, $t / C$ oranının akış yapısı üzerindeki etkisinin oldukça önemli olduğunu göstermektedir. Düşük hücum açıları için, $t / C$ oranı arttıkça, kanat üzerindeki girdap yapısının güçlendiği görülmektedir. Öte yandan, düşük $t / C$ oranındaki kanatlarda yüksek $t / C$ oranlarına sahip kanatlara kıyasla, 3 boyutlu yüzey ayrılmasıyla daha yüksek hücum açılarında karşılaştığı söylenebilir. Bu 
sonuçlar, yüksek $t / C$ oranına sahip kanatların düşük hücum açılarında daha yüksek boyutsuz kaldırma kuvveti katsayılarına, CL, ulaştığı ancak düşük $t / C$ oranına sahip kanatlar için, boyutsuz kaldırma kuvveti katsayısının en yüksek ve yüksek hücum açılarında ortaya çıktığı kuvvet ölçüm sonuçları ile desteklenmektedir. Bu sonuçlar gösteriyor ki, düşük $t / C$ oranındaki kanatlar perdövitese girmeye karşı daha dayanıklıdırlar. Boyutsuz kaldırma kuvveti katsayısı ve sürtünme katsayısı oranı, $\mathrm{C}_{\mathrm{L}} / \mathrm{C}_{\mathrm{D}}$, incelendiğinde ise, $\mathrm{t} / \mathrm{C}$ oranındaki artış, $\mathrm{C}_{\mathrm{L}} / \mathrm{C}_{\mathrm{D}}$ değerinin kayda değer bir miktarda düşüşe neden olduğunu görülmektedir.

Anahtar Kelimeler: Delta kanad1, düşük süpürme, aerodinamik kuvvetler, kanat kalınlığı, kalınlık-veter oranı 
To my parents 


\section{ACKNOWLEDGMENTS}

I would like to express my sincerest appreciation to my thesis supervisor, Assoc. Prof. Dr. Mehmet Metin Yavuz for his guidance and support. Dr. Yavuz is one of the friendliest supervisor with an extremely open-mind and his demeanor and positive attitude have shaped a pattern for my life.

Words cannot express how grateful I am to my parents Mahin Chamani and Rahim Sharifi for their irreplaceable unfailing support and continuous encouragement throughout my years of study. This accomplishment would not have been possible without them.

I must express my profound gratitude to my colleagues Burcu Ramazanlı and Kayacan Kestel for sharing their experimental experience. Their impact on this thesis is substantial and unforgettable.

I would like to thank my friends Burak Gülsaçan, Gizem Şencan, and Cenk Çetin for their support and pioneering studies in Fluid Mechanics Lab which pave the way for further investigations.

The technical assistance of Mr. Mehmet Özçiftçi and Mr. Servet Şehirli are gratefully acknowledged. 


\section{TABLE OF CONTENTS}

ABSTRACT

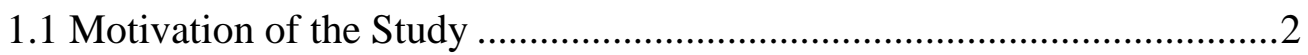

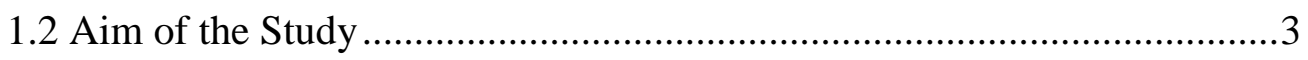

1.3 Structure of the Thesis ...................................................................... 3

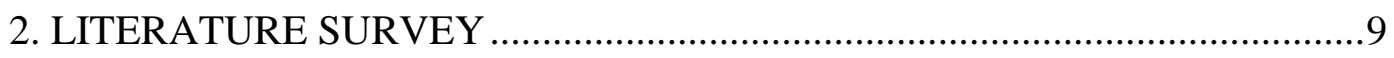

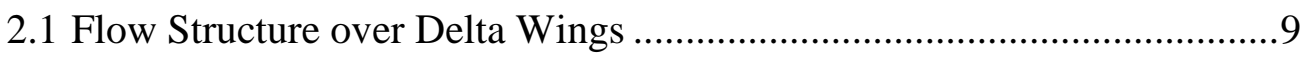

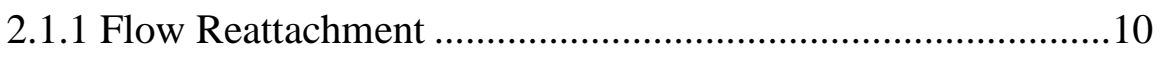

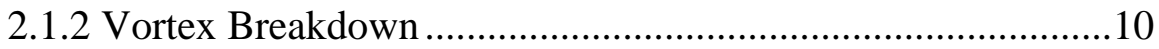

2.1.3 Flow Control Techniques...................................................11

2.2 Effect of Thickness-To-Chord Ratio....................................................13

3. EXPERIMENTAL SET-UP AND MEASUREMENT TECHNIQUES ..........29

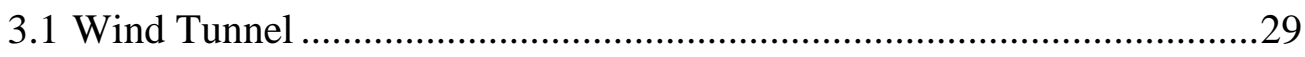

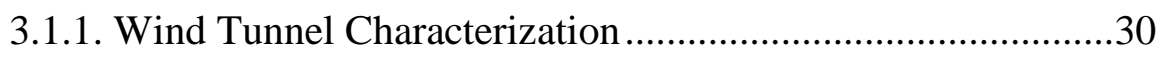


3.2 Flow Measurement Techniques............................................................ 30

3.2.1. Laser-Illuminated Flow Visualization ................................... 30

3.2.2. Surface Pressure Measurement.............................................. 30

3.2.3. Particle Image Velocimetry (PIV) Measurements .................31

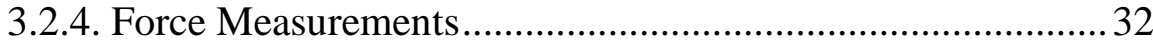

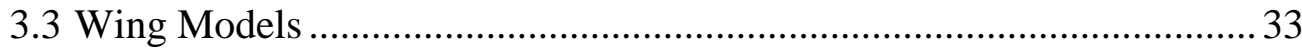

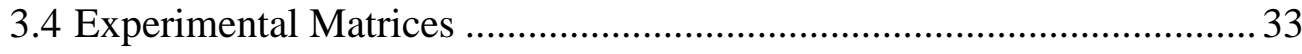

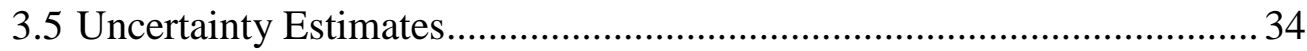

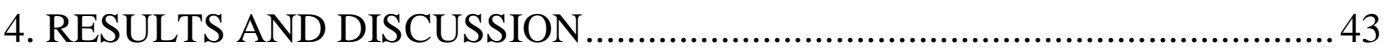

4.1 The Results of Surface Flow Visualizations ......................................... 43

4.2 The Results of Surface Pressure Measurements...................................... 47

4.3 The Results of Crossflow PIV Measurements ....................................... 52

4.4 The Results of Force Measurements .....................................................58

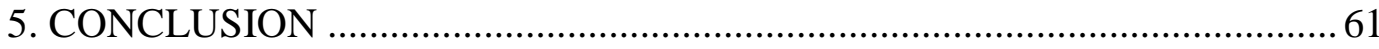

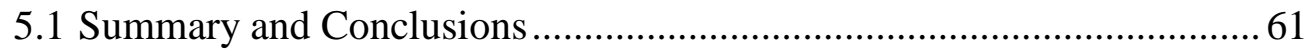

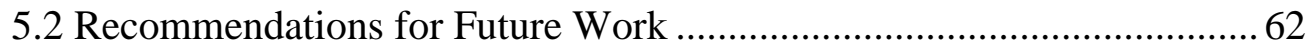

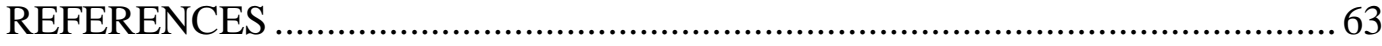

APPENDICES

A. CODE FOR CALCULATING RMS VALUES OF VELOCITY

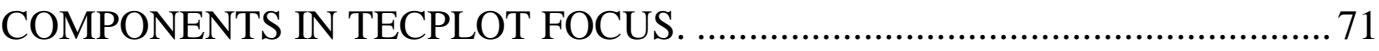

B. SOURCE CODES FOR PRESSURE COEFFICIENT UNCERTAINITY

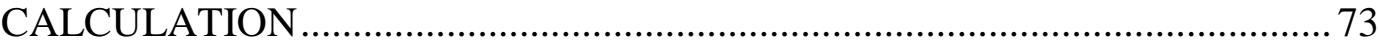

C. FORMULAS FOR LIFT AND DRAG COEFFICIENTS AND CORROSPONDING UNCERTAINITY CALCULATIONS............................ 75 
D. LASER ILLUMINATED SURFACE FLOW SMOKE VISUALIZATIONS FOR THICKNESS-TO-CHORD RATIOS OF 0.033, 0.05, 0.10, 0.15 FOR $\mathrm{RE}=10000 \mathrm{AT} \alpha=6^{\circ}, 10$ AND FOR RE=10000, AND 15000 AT $\alpha=14^{\circ} \ldots \ldots \ldots . .77$

F. THE DIMENSIONLESS PRESSURE DISTRIBUTION - Cp WITH RESPECT TO DIMENSIONLESS HALF SPAN FOR THICKNESS-TO-CHORD RATIOS OF $0.033,0.05,0.10,0.15$ FOR RE $=35000,75000,100000,125000$ AT $\alpha=16^{\circ}$ AND FOR RE $=35000,100000$ AT $\alpha=18^{\circ}$ .83 


\section{LIST OF TABLES}

\section{TABLES}

Table 1 Relative uncertainty values for the pressure measurements at the peak values for $\mathrm{t} / \mathrm{C}$ ratios of $0.033,0.05,0.10$, and 0.15 at $\mathrm{Re}=35000$ and 100000 for

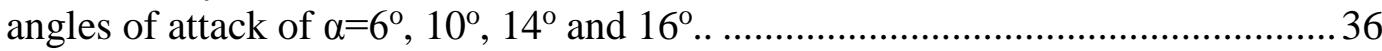

Table 2 Maximum uncertainty values for the PIV measurements at $\mathrm{Re}=35000$ for

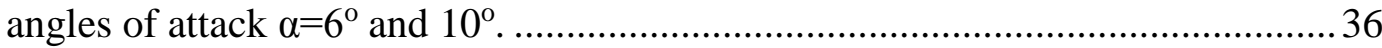

Table C.1 Corresponding uncertainty values of force measurements for wing with $t / C=15 \%$ at different angle of attacks. ..................................................... 76 


\section{LIST OF FIGURES}

\section{FIGURES}

Figure 1.1 Schematic of delta wing geometrical characteristics..........................5

Figure 1.2 Velocity contours of a delta wing in different cross sections [9]...........5

Figure 1.3 (a) Delta wing vortex formation along with sectional pressure distribution and (b) vortex breakdown [10]..................................................... 6

Figure 1.4 Near surface flow structure and streamlines for a non-slender delta wing at $\alpha=5^{\circ}$ (top) and $8^{\circ}$ (bottom) [11].

Figure 1.5 Schematic vortical flow patterns (a) over non-slender delta wings with reattachment and (b) over slender delta wings with no reattachment [2] ...............8

Figure 1.6 Dual vortex structure over non-slender delta wings [12] ....................

Figure 2.1 illustration of discerete vortices emanating from the leading edge [16].

Figure 2.2 Laser light sheet illumination of primary vortices and the discrete vortices that are feeding them [17].

Figure 2.3 Flow visualization of dual vortex structure [21].

Figure 2.4 Schematic of vortical flow for non-slender delta wing with the presence of vortex Reattachment (top) and slender delta wing without the reattachment (bottom) [21]

Figure 2.5 Skin friction lines and the corrosponding secondary separation and reattachment lines [22].

Figure 2.6 Smoke visualization results at $R e=1 \times 10^{4}$ for $\alpha=3^{\circ}$ and $8^{\circ}$ for 45 deg swept delta wing [23].

Figure 2.7 $C L$ versus $\alpha$ for rigid wings with the change of sweep angle [51]......21

Figure 2.8 Comparison of $C L$ for rigid and flexible wings with 60 deg sweep angle [51].

Figure 2.9 Shape of airfoils with different relative thickness values (top), and corrosponding (a) lift and (b) drag coefficient values (bottom) [58]. 
Figure 2.10 Sectional lift and drag coefficients versus angle of attack during a constant pitch rate maneuver for airfoils with thickness ratios of $t / C=0.09$,

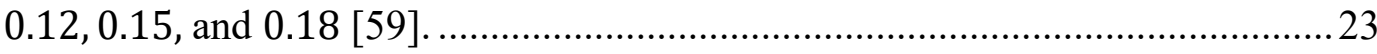

Figure 2.11 Variation of lift and normal force coefficients slopes versus $t / C$ ratio [61]

Figure 2.12 Angle of attack of maximum lift-to-drag ratio versus $t / C$ ratio [61].24

Figure 2.13 Variation of maximum lift-to-drag ratio versus $t / C$ ratio for different

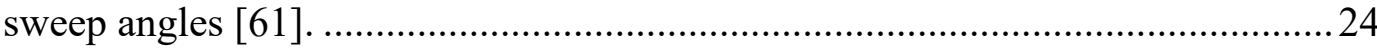

Figure 2.14 Thickness and leading edge geometry effects on primary vortex

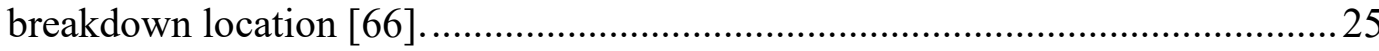

Figure 2.15 Effect of wing thickness-to-chord ratio on drag divergence, Mach

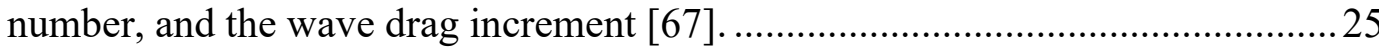

Figure 2.16 Wing thickness effect on (a) dimensionless flutter speed and (b) dimensionless flutter frequency, versus dimensionless span location of store [68].

Figure 2.17 Wing thickness effect on $C_{L}$ and $C_{D}$ values [9]...........................26

Figure 2.18 Lift-to-drag ratio versus angle of attack for thick and thin wing models [9]

Figure 2.19 Difference between thin and thick wing in velocity contours at (a) $\alpha=$

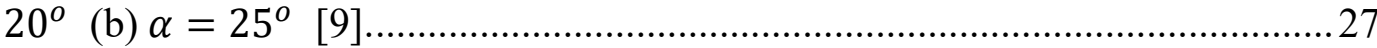

Figure 2.20 Effect of $t / C$ ratio on patterns of time-averaged streamlines [8].....28

Figure 3.1 View from (a) wind tunnel facility and (b) test section. 37

Figure 3.2 Tunnel fan power and turbulence intensity versus freestream velocity

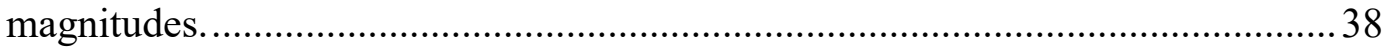

Figure 3.3 Schematic representation of Surface flow visualization. .....................38

Figure 3.4 Schematics representation of crossflow PIV laser sheet and pressure

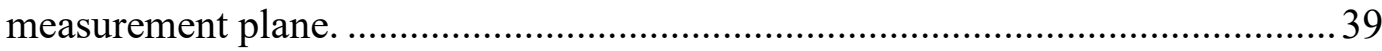

Figure 3.5 Schematic representation of force measurement system and corresponding normal to surface, $\mathrm{N}$, and axial, A, aerodynamic forces................39

Figure 3.6 Calibration of full bridge strain gauges..........................................40 
Figure 3.7 Schematic of: (a) geometrical dimensions and pressure and smoke holes of the wing models and (b) cross-sectional view of the wings and corresponding $t / C$ ratios.

Figure 3.8 Experimental matrices for (a) surface laser illuminated flow smoke visualizations (b) pressure measurements and (b) PIV measurements.

Figure 4.1 Laser-illuminated surface flow smoke visualizations of $t / C=$ $0.033,0.05,0.10$ and 0.15 for $R e=1.5 \times 10^{4}$ at angle of attack of $\alpha=6 \mathrm{deg}$. 45

Figure 4.2 Laser-illuminated surface flow smoke visualizations of $t / C=$ $0.033,0.05,0.10$ and 0.15 for $R e=1.5 \times 10^{4}$ at angle of attack of $\alpha=10 \mathrm{deg}$. .

Figure 4.3 The dimensionless pressure distribution $-\mathrm{C}_{\mathrm{p}}$ with respect to dimensionless half span for $\mathrm{t} / \mathrm{C}=0.033,0.05,0.10$, and 0.15 for $\mathrm{Re}=3.5 \times$ $10^{4}$ at angles of attack of $\alpha=6,10,14$, and 16 degrees.

Figure 4.4 The dimensionless pressure distribution $-C_{p}$ with respect to dimensionless half span for $\mathrm{t} / \mathrm{C}=0.033,0.05,0.10$, and 0.15 for $\mathrm{Re}=1 \times 10^{5}$ at angles of attack of $\alpha=6,10,14$, and 16 degrees.

Figure 4.5 The time-averaged velocity vectors $\langle\mathrm{V}\rangle$ and constant contours of nondimensional axial vorticity $\langle\omega \mathrm{C} / \mathrm{U}\rangle$ at angles of attack $\alpha=10$ (left column) and 14 (right column) degrees and $\mathrm{Re}=3.5 \times 10^{4}$ for $\mathrm{t} / \mathrm{C}=0.02,0.033,0.05,0.10$, and 0.15: $[|\langle\omega \mathrm{C} / \mathrm{U}\rangle|]_{\min }=4, \Delta[|\langle\omega \mathrm{C} / \mathrm{U}\rangle|]=4$.

Figure 4.6 The time-averaged velocity vectors $\langle\mathrm{V}\rangle$ and constant contours of nondimensional axial vorticity $\langle\omega \mathrm{C} / \mathrm{U}\rangle$ at angles of attack $\alpha=10$ (left column) and 14 (right column) degrees and $\mathrm{Re}=1 \times 10^{5}$ for $\mathrm{t} / \mathrm{C}=0.02,0.033,0.05,0.10$, and 0.15: $[|\langle\omega \mathrm{C} / \mathrm{U}\rangle|]_{\min }=4, \Delta[|\langle\omega \mathrm{C} / \mathrm{U}\rangle|]=4$.

Figure 4.7 Contours of constant non-dimensional rms of horizontal velocity $\mathrm{v}_{\mathrm{rms}} / \mathrm{U}$ and vertical velocity $\mathrm{w}_{\mathrm{rms}} / \mathrm{U}$ at angles of attack $\alpha=10$ (left column) and 14 (right column) degrees and $\mathrm{Re}=3.5 \times 10^{4}$ for $\mathrm{t} / \mathrm{C}=0.02,0.033,0.05,0.10$, and 0.15: $\left[\left|\left\langle\mathrm{v}_{\mathrm{rms}} / \mathrm{U}\right\rangle\right|\right]_{\min }=0.1, \quad\left[\left|\left\langle\mathrm{w}_{\mathrm{rms}} / \mathrm{U}\right\rangle\right|\right]_{\min }=0.1, \Delta\left[\left|\left\langle\mathrm{v}_{\mathrm{rms}} / \mathrm{U}\right\rangle\right|\right]=0.02$, $\Delta\left[\left|\left\langle\mathrm{w}_{\mathrm{rms}} / \mathrm{U}\right\rangle\right|\right]=0.02$.

Figure 4.8 Lift coefficient $C_{L}$ (top), Drag coefficient $C_{D}$ (middle), and Lift-to-Drag ratio $C_{L} / C_{D}$ (bottom) with respect to angle of attack for $t / C=$ $0.02,0.033,0.05,0.10$, and 0.15 at $\mathrm{Re}=3.5 \times 10^{4}$.

Figure D.1 Laser-illuminated surface flow smoke visualizations of $\mathrm{t} / \mathrm{C}=$ $0.033,0.05,0.10$ and 0.15 for $\operatorname{Re}=1 \times 10^{4}$ at angle of attack of $\alpha=6 \mathrm{deg}$. ...78 
Figure D.2 Laser-illuminated surface flow smoke visualizations of $\mathrm{t} / \mathrm{C}=$ $0.033,0.05,0.10$ and 0.15 for $\operatorname{Re}=1 \times 10^{4}$ at angle of attack of $\alpha=10 \mathrm{deg}$. .. 79

Figure D.3 Laser-illuminated surface flow smoke visualizations of $\mathrm{t} / \mathrm{C}=$ $0.033,0.05,0.10$ and 0.15 for $\operatorname{Re}=1 \times 10^{4}$ at angle of attack of $\alpha=14 \operatorname{deg} \ldots .80$

Figure D.4 Laser-illuminated surface flow smoke visualizations of $\mathrm{t} / \mathrm{C}=$ $0.033,0.05,0.10$ and 0.15 for $\operatorname{Re}=1.5 \times 10^{4}$ at angle of attack of $\alpha=14 \mathrm{deg} .81$

Figure D.5 Laser-illuminated surface flow smoke visualizations of $\mathrm{t} / \mathrm{C}=$ $0.033,0.05,0.10$ and 0.15 for $\operatorname{Re}=2 \times 10^{4}$ at angle of attack of $\alpha=14 \operatorname{deg} \ldots .82$

Figure F.1 The dimensionless pressure distribution $-C_{p}$ with respect to dimensionless half span for $t / C=0.033,0.05,0.10$, and 0.15 for $\operatorname{Re}=$ $35000,75000,100000$, and 125000 at angles of attack of $\alpha=16$ degree. .......83

Figure F.2 The dimensionless pressure distribution $-C_{p}$ with respect to dimensionless half span for $\mathrm{t} / \mathrm{C}=0.033,0.05,0.10$, and 0.15 for $\operatorname{Re}=$ 35000 and 100000 at angles of attack of $\alpha=18$ degree. 84 
NOMENCLATURE

\section{Greek Symbols}

$\begin{array}{ll}\Lambda & \text { Sweep angle } \\ \alpha & \text { Angle of attack } \\ \rho & \text { Fluid density } \\ v & \text { Kinematic viscosity of air }\end{array}$

\section{Latin Symbols}

C Root chord length

S Half-span at $\mathrm{x} / \mathrm{C}=0.5$

Re Reynolds number based on chord length

U Free stream velocity

V Velocity vector

u Streamwise velocity

w Normal to surface velocity component

$\mathrm{v} \quad$ Transverse velocity component

$\omega \quad$ Vorticity

$\mathrm{x} \quad$ Chordwise distance from wing apex

y Spanwise distance from wing root chord

$\delta / C \quad$ Effective grid size

t/C Thickness-to-chord ratio

$p \quad$ Surface pressure

$\bar{p} \quad$ Average pressure

$p_{\infty} \quad$ Static pressure of the flow

$p_{d y n} \quad$ Dynamic pressure of the flow

$C_{p} \quad$ Dimensionless pressure coefficient

$\mathrm{t} \quad$ Thickness of the wing 


$\begin{array}{ll}\text { PIV } & \text { Particle Image Velocimetry } \\ i_{r m s} & \text { Root mean square of a variable } \mathrm{i} \\ \omega_{i} & \text { Uncertainty estimate of a variable } \mathrm{i} \\ u_{i} & \text { Relative uncertainty estimate } \\ \mathrm{M} 1 & \text { Measured strain in full bridge } 1 \\ \mathrm{M} 2 & \text { Measured strain in full bridge } 2 \\ \mathrm{M} 3 & \text { Measured strain in full bridge } 3 \\ \mathrm{~N} & \text { Normal to surface aerodynamic force } \\ \mathrm{A} & \text { Axial aerodynamic force } \\ \mathrm{A} \text { Ref } & \text { Wing surface area } \\ \mathrm{X} & \text { Calibration coefficient to convert force to measured strain } \\ C_{L} & \text { Lift coefficient } \\ C_{L m a x} & \text { maximum value of Lift coefficient } \\ C_{D} & \text { Drag coefficient } \\ C_{L} / C_{D} & \text { Lift-to-Drag ratio }\end{array}$




\section{CHAPTER 1}

\section{INTRODUCTION}

In recent decades, the prevailing usage of delta wings in the design of unmanned air vehicles (UAVs), micro air vehicles (MAVs), and fighter jets motivated extensive studies on the flow control and flow characterization of these planforms [1], [2]. The investigations in this area date back to 1960's [3], however supplementary investigations are necessary to elucidate the flow topology and aerodynamics and to reduce the flow instabilities and enhance the overall performance of delta wings during steady flights and maneuvers.

Delta wings are basically categorized according to their sweep angle into two groups of non-slender (with less than 55 deg sweep angle) and slender (with higher than $55 \mathrm{deg}$ sweep angle) delta wings. Figure 1.1 shows the dimensional characteristics of delta wings. Non-slender delta wings have significant superiority in terms of high maneuverability and low structural-weight-to-takeoff-weight ratio. However, compared to slender delta wings, relatively less investigations are dedicated to flow structure characterization, flow control, and determination of unsteady behaviors on non-slender delta wings.

The flow over delta wing separates from the leading edge and rolls up into a coherent vortex structure, leading edge vortex, which is one of the main contributors to the lift force due to the low pressures in its core. Figure 1.2 represents the velocity contours over a delta wing and the corresponding vortex structure. At adequately high incidences, vortex breakdown appears on the planform in proximity to the trailing edge, and moves upstream toward the apex as the attack angle increases [4]. Figure 1.3 represents the vortex formation and breakdown along with the typical minus pressure coefficient, $-C_{p}$, distribution in the presence of vortex flow. Along 
with the movement of vortex breakdown location toward apex, in non-slender wings, back flow region is apparent near the leading edge interacting with the trailing edge and initiates a swirl, three-dimensional separated region, on the planform with increase in angle of attack [5]. A schematic of this phenomenon is represented in Figure 1.4. Non-slender and slender delta wings have some differences on their flow structure. One of the differences is the proximity of the vortex structure to the wing planform and reattachment to the wing surface in nonslender delta wings unlike the slender ones [2]. A schematic of this phenomenon is demonstrated in Figure 1.5. This reattachment point moves toward the wing centerline along with the increase in attack angle and just after it reaches the centerline, the flow experiences the three dimensional separation. The consequence of the vortical flow proximity to the wing surface is the interaction between the vortex and boundary layer that leads to the appearance of secondary and even tertiary vortices [6], which can intensify the sensitivity of flow field to Reynolds number [7]. As a result, the unsteady behaviors of the flow, which are also present in slender delta wings, are intensified by these distinct features of non-slender delta wings. Figure 1.6 shows a schematic of the presence of secondary vortices in the flow field of non-slender delta wing.

\subsection{Motivation of the Study}

Delta wings play a major rule in the design of Micro air vehicles (MAV), Unmanned Air Vehicles (UAV) and Unmanned Combat Air Vehicles (UCAV), and supersonic aircrafts. Therefore, enlightenment of the various aspects of the flow over these planforms is vital for the enhancement of the future air vehicles design. Wing thickness ratio is one of the significant parameters which can limit the various operational capabilities of the air vehicles, such as payload, armament, loading configurations, and etc.

For non-slender delta wings, a very few studies have addressed the thickness effect on aerodynamic forces and flow structure. Recently, Gülsaçan et al. [8] investigated 
the effect of $t / C$ ratio on flow structure of a 35 deg swept delta wing using wing models with $t / C$ ratios of $4.75,9.5,14,25$, and $19 \%$, and concluded that effect of $t / C$ ratio on flow structure of delta wings is as significant as the effect of attack angle, and vortex breakdown and three dimensional separation occur in relatively lower angles of attack as the $t / C$ ratio increases. Furthermore at low angle of attacks, the higher $t / C$ ratio wings experienced stronger vortex structure. However, further studies are required to check the coherency of the results of Gülsaçan et al. [8] with a different delta wing with higher sweep angle and for thinner wing models with $t / C$ ratios less than $4.75 \%$. Furthermore, addition of force measurement results is necessary to check the applicability of flow control by $t / C$ ratio variation and also to derive a solid conclusion about the $t / C$ ratio effect on both the flow structure and aerodynamics of non-slender delta wings combined.

\subsection{Aim of the Study}

The aim of present research is to characterize the $t / C$ ratio effect on both the flow structure and the aerodynamic forces of a 45 deg swept sharp-edged delta wing. Five different delta wing models with $t / C$ ratios of $2,3.3,5,10$, and $15 \%$ have been used and experiments have been conducted at Reynolds numbers of $R e=1.5 \times$ $10^{4}$ to $1 \times 10^{5}$, for the attack angles ranging from 0 to 30 degrees. Experiments were performed in low-speed wind tunnel using laser illuminated smoke visualization, surface pressure measurements, particle image velocimetry, and force measurements.

\subsection{Structure of the Thesis}

The present thesis consists of five main chapters. Chapter 1 provides introductory information on the flow structure over delta wings, and continues with brief description of motivation and aim of study of current thesis.

In Chapter 2, the literature survey is provided. First, the detailed description of flow structure is included and then the various control techniques are mentioned. Finally, 
the previous results on thickness-to-chord ratio effect with a specific emphasis on non-slender delta wings are provided.

Chapter 3 involves the measurement techniques along with the experimental set-up and matrix of the present study.

Chapter 4 discusses the experimental results on flow structure and aerodynamic forces. It starts with laser illuminated smoke visualizations and continues with surface pressure measurements and then particle image velocimetry results, and finishes with force measurement data.

Finally, Chapter 5 includes the main conclusions of the present study and represents the recommendations for the future work. 


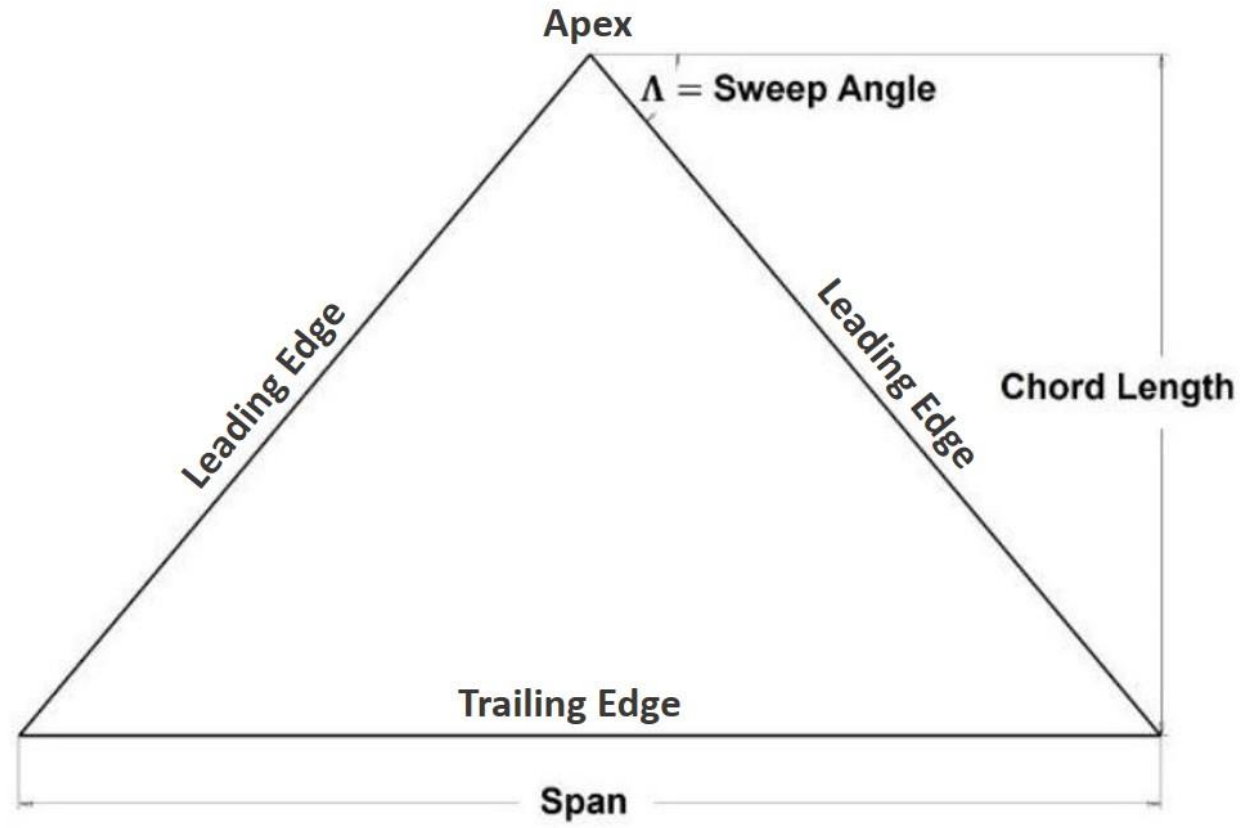

Figure 1.1 Schematic of delta wing geometrical characteristics.

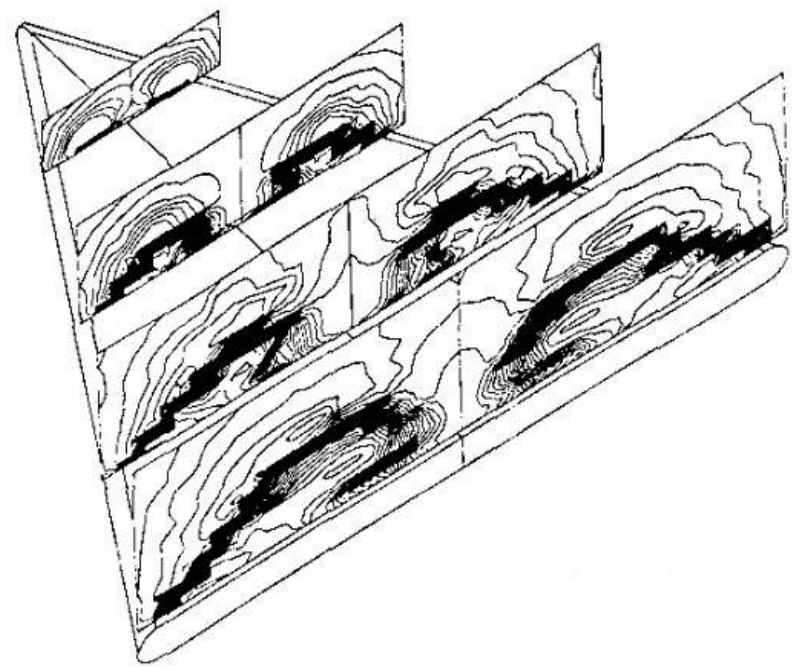

Figure 1.2 Velocity contours of a delta wing in different cross sections [9]. 
a
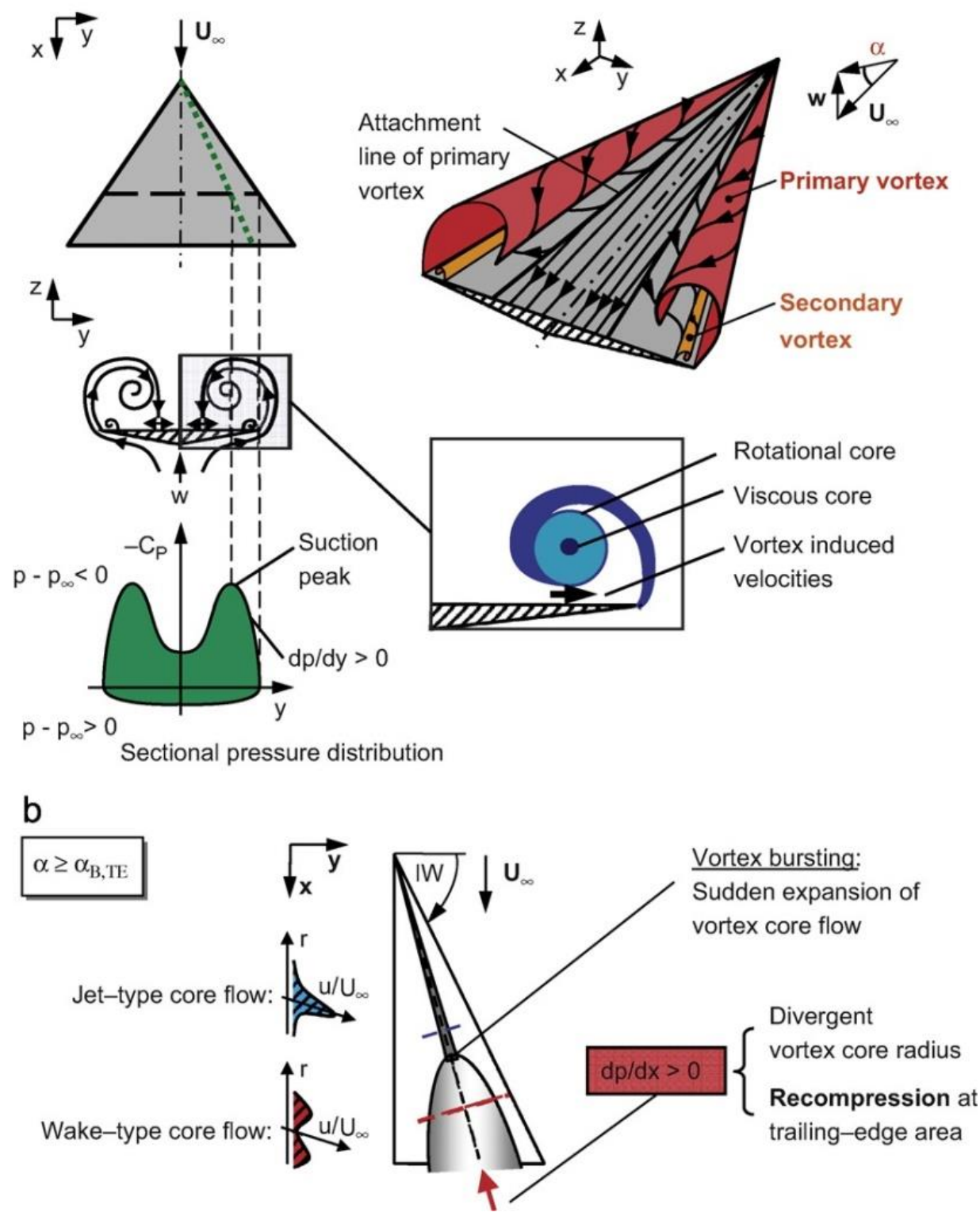

Figure 1.3 (a) Delta wing vortex formation along with sectional pressure distribution and (b) vortex breakdown [10]. 

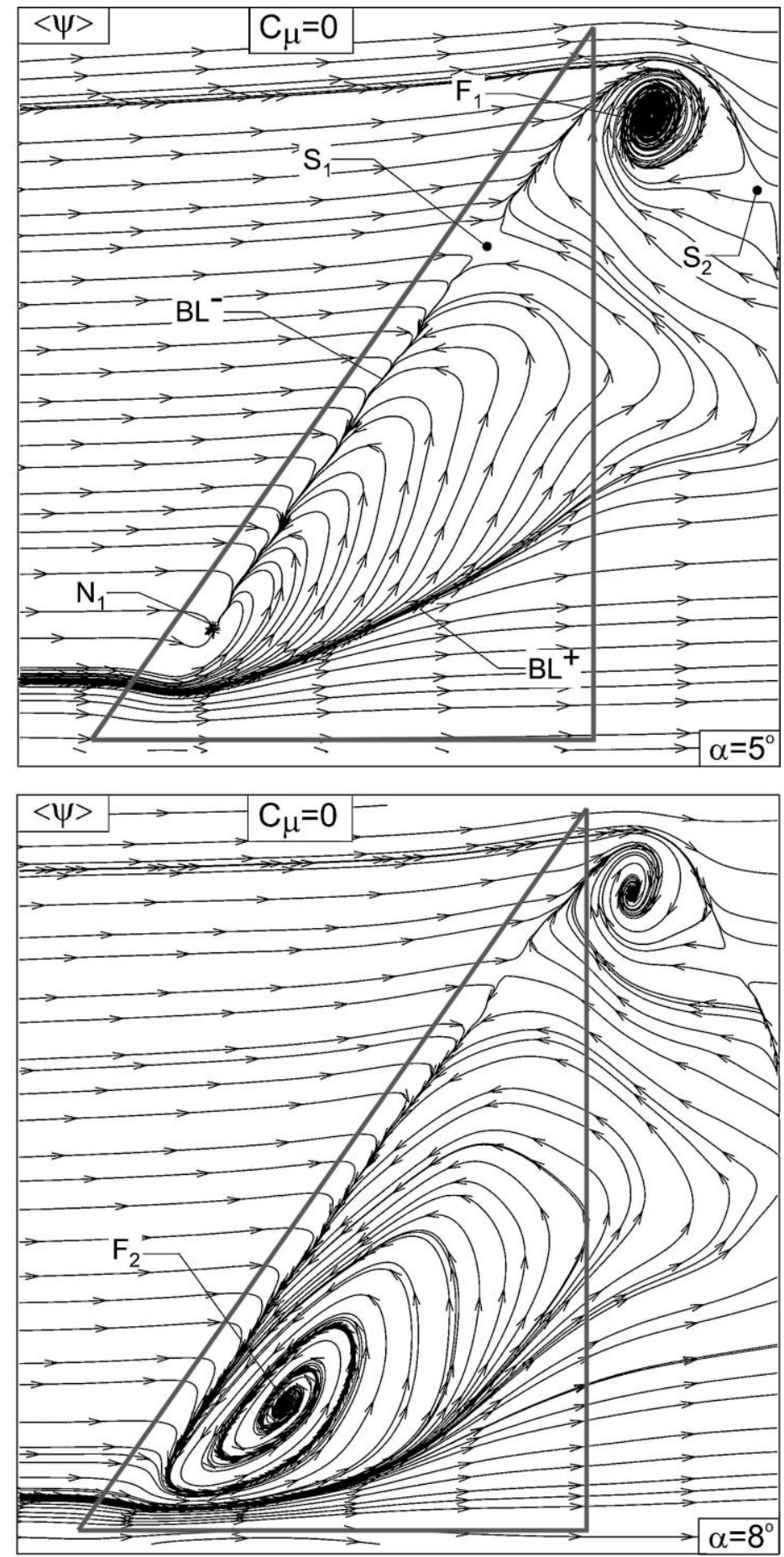

Figure 1.4 Near surface flow structure and streamlines for a non-slender delta wing at $\alpha=5^{\circ}$ (top) and $8^{\circ}$ (bottom)[11]. 


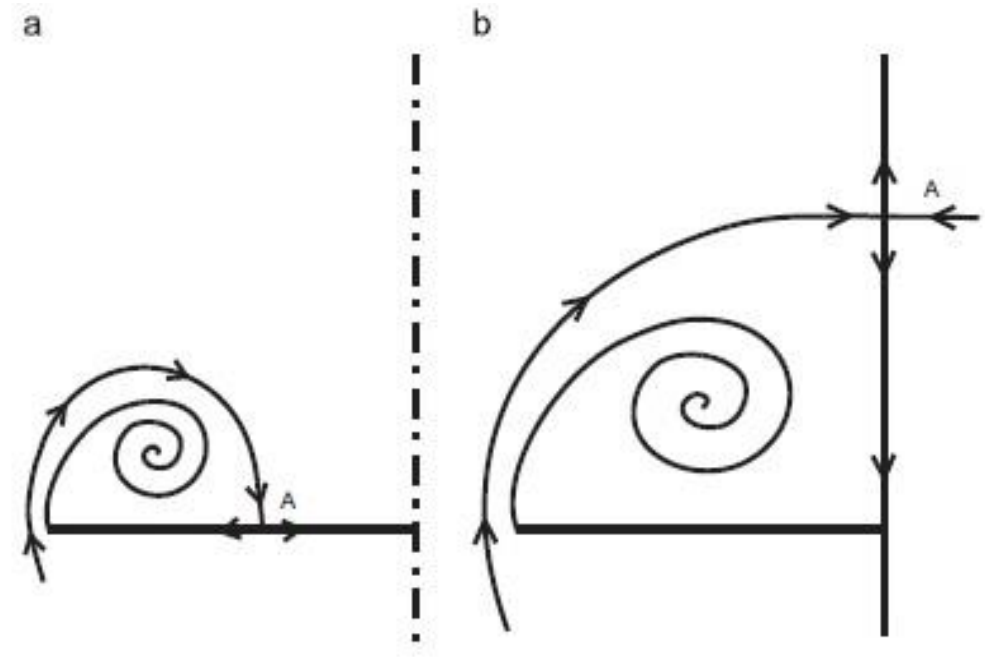

Figure 1.5 Schematic vortical flow patterns (a) over non-slender delta wings with reattachment and (b) over slender delta wings with no reattachment [2].

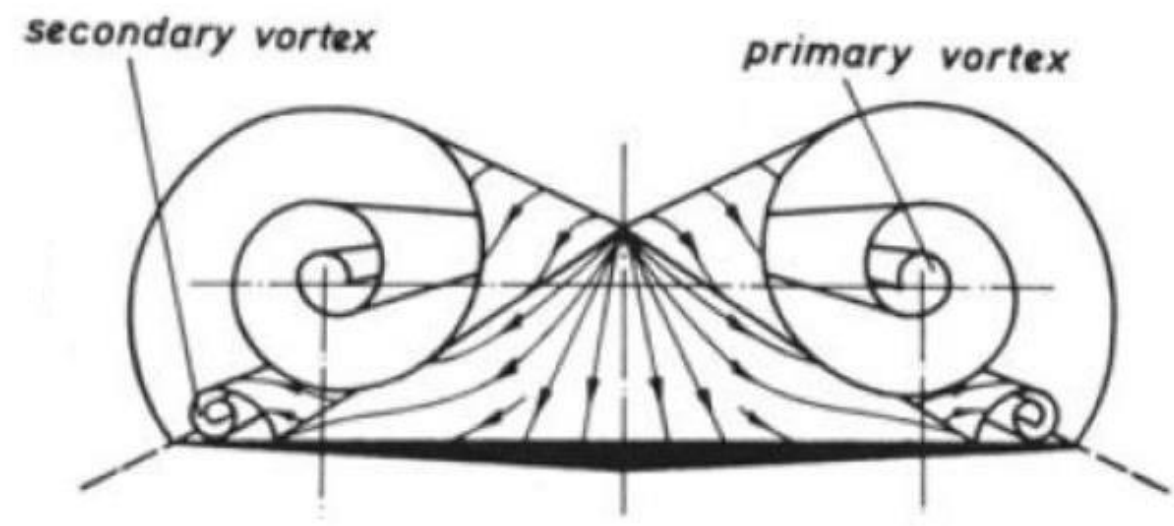

Figure 1.6 Dual vortex structure over non-slender delta wings [12]. 


\section{CHAPTER 2}

\section{LITERATURE SURVEY}

This chapter summarizes the essential literature related to flow physics over delta wings with a particular focus on non-slender delta wings. Furthermore, various flow control techniques are reviewed and previous investigations related to the effect of thickness-to-chord ratio are summarized.

\subsection{Flow Structure over Delta Wings}

Flow over delta wings is dominated by two counter rotating vortices fed by discrete vortices separating from the leading edge [13]-[15]. This phenomenon is illustrated by Gad-el-hak and Blackwelder [16], as represented in Figure 2.1. Nelson and Pelletier [17] demonstrated the convolution of the discrete vortices into coherent vortex structure, as shown in Figure 2.2. The velocities inside the vortex core are much higher than the free stream velocity, and according to the Bernoulli's principle this results in lower pressure in the suction side of the planform. In slender delta wings the velocities inside the vortex core can rise up to 4-5 times of the free stream velocities [7], and according to Polhamus [18], vortex lift can induce as much as $50 \%$ of total lift force in slender delta wings.

Despite the general similarity in flow topology between non-slender and slender delta wings, there are some notable differences. One of the major differences is that in non-slender delta wings vortex core forms closer to the wing surface compared to slender wings [19]. This causes the interaction of the vortex structure and boundary layer which leads to creation of secondary vortex structure with opposite rotational direction [6] in low attack angles and Reynolds numbers [20]. This dual vortex structure is observed both in numerical [6] and experimental [21] 
investigations. Figure 2.3 represents the illuminated flow field with a dual vortex structure over a non-slender delta wing.

\subsubsection{Flow Reattachment}

Another difference in flow structure between non-slender and slender delta wings is the flow reattachment. The separated shear layer over non-slender delta wings experiences the reattachment to the wing surface outboard the wing centerline [7], and the location of this reattachment point approaches the wing center line as the attack angle increases. However, for slender delta wings, this phenomenon is only observed in very low incidences, and its control is difficult [2]. Figure 2.4 demonstrates the corresponding difference of non-slender and slender wings in terms of flow reattachment. Woodiga and Liu [22] visualized skin friction fields over a 65 deg swept delta wing using luminescent oil-film skin friction meter and identified reattachment line and secondary vortex separation line as represented in Figure 2.5. Zharfa et al. [23] stated that in the presence of leading edge vortex, highest velocity fluctuations occur between reattachment line and vortex core. Taylor and Gursul [24] investigated the buffeting flow in low swept delta wings and observed the largest velocity fluctuation near the wing surface along the reattachment line. They concluded that in prestall condition shear layer reattachment is the main source of buffeting.

\subsubsection{Vortex Breakdown}

Vortex breakdown can be identified as sudden expansion of vortex structure. For delta wings, this phenomenon takes place over the wing planform at sufficiently high incidences. Delery [25] reviewed various aspects of vortex breakdown and proposed the adverse pressure gradient induction as a cause of rapid deceleration in the axial motion and vortex breakdown. Vortex breakdown types are categorized into seven different kinds [26], three of which are more common over delta wings, spiral, double helix, and bubble type. Sarpkaya [27],[28] investigated the vortex breakdown on cylindrical tubes and stated that the type and the location of the 
breakdown is dependent on Reynolds number. Payne et al. [29] visualized vortex breakdown for series of slender delta wings using smoke flow visualization and concluded that at a given angle of attack as sweep angle increases the breakdown location moves downward to the trailing edge. In non-slender delta wings, as the attack angle increases, the vortex breakdown location advances toward apex and the reattachment point moves toward centerline [23]. Meanwhile, swirl flow emerges over the wing suction side, and further increase in angle of attack enlarges it and moves its center toward trailing edge [5]. Zharfa et al. [23] demonstrated the effect of increase in angle of attack at flow reattachment location and swirl flow using flow smoke visualization as shown in Figure 2.6. The emergence of swirl flow is a sign of three dimensional separation in the flow and its presence is an indication of prestall condition in the flow field. Vortex breakdown and three dimensional separation reduce the lift force and intensify the unsteady behavior of the flow field [30], therefore preventing the vortex from breakdown is the main objective of most of the investigations in this area.

\subsubsection{Flow Control Techniques}

Various active and passive flow control techniques have been implemented to enhance the flow reattachment and vortex formation, and also to delay or eliminate the vortex breakdown, flow separation, and stall. These techniques are categorized into two groups of active and passive flow control techniques. For active flow control techniques such as blowing, suction, and unsteady excitation an additional energy is necessary, whereas passive techniques like bleeding does not require it [2].

Wood and Roberts [31] conducted one of the earliest studies on active flow control. They implemented tangential leading edge blowing to control vortical lift over a 60 deg swept delta wing. They delayed vortex breakdown significantly and achieved approximately $30 \%$ higher normal force coefficient. This technique also was used by Wood et al. [32] to control the asymmetric vortical flow over a low swept delta wing at high incidences. Gu et al. [33] examined the flow control over a slender 
delta wing by applying steady blowing, steady suction, and alternate suctionblowing from the leading edge, and maximum downward movement of breakdown location was achieved by alternate suction-blowing. Trailing edge jet blowing first was applied by Helin and Watry [34] to move the bursting location of the leading edge vortices downward. Investigations over flow control of delta wings by trailing edge blowing were continued by steady [11], [35] and unsteady blowing [36]. Furthermore, Shih and Ding [37], and Wang et al. [38] examined the effect of downward angle of the trailing edge blowing. Suction near the separation point was another technique that was implemented to control the location and direction of the primary vortices [39]. Near core blowing was investigated by Kuo an Lu [40] and Guillot et al. [41], and Liu et al. [42] used closed-loop active flow controller to derive the efficient injection cycles of the controlling jets along the vortex core. Mitchell et al. [43] moved the vortex breakdown location downward as $20 \%$ of wing chord length utilizing along the core blowing. Several studies were dedicated to control the flow structure over delta wings by periodic blowing from the leading edge [44], [45], and periodic blowing and suction [46]-[48]. Passive bleeding along the leading edge was introduced as an effective method to delay vortex breakdown by Çelik et al. [49], and they reported the configuration of the bleeding holes as an important parameter in vortex breakdown control.

Geometrical characteristics of delta wings, including sweep angle, wing flexibility, leading edge geometry, and thickness-to-chord ratio $t / C$, have significant influence on flow structure and aerodynamic performance. Sweep angle effect was extensively studied since 1964 [13], and Gursul and Batta [50] employed variable sweep angle delta wing to particularly control the location of vortex breakdown. Yang et al. [51] investigated the effect of wing flexibility on aerodynamic characteristics of delta wings with different sweep angles ranging from 25 to 65 degrees. Figure 2.7 demonstrates the effect of sweep angle on lift coefficient of rigid wing models, where $C_{\text {Lmax }}$ and stall angle of attack increase with increasing sweep angle. Although, Figure 2.8 represents the effect of wing flexibility on lift 
coefficient curve for 60 deg swept delta wing, where $C_{\text {Lmax }}$ increases for flexible wing but the stall angle of attack is unaffected by wing flexibility. Taylor et al. [52] explored the lift enhancement on a series of flexible delta wings where they observed improvement in lift and stall angle compared to rigid models. Effect of leading edge modifications, including sinusoidal [53]-[55], and bioinspired [56], on overall flow structure on delta wings have recently been investigated. Chen et al. [54],[55] delayed the leading edge vortex breakdown and stall angle of attack, and enhanced the post-stall lift coefficient using sinusoidal leading edge. Furthermore, they increased the stall angle of attack by increasing the amplitude or decreasing the wave length of sinusoidal leading edge profile. Wang and $\mathrm{Lu}$ [57] examined the effect of leading edge bevel angle on lift and drag forces of a 50 deg swept delta wing where they reported that the wing with leeward bevel induces significantly higher lift along with higher stall angle and reduced drag compared to the wing with windward bevel.

\subsection{Effect of Thickness-To-Chord Ratio}

Wing thickness plays a significant role in determination of overall performance and characteristics of air vehicles. Thickness effect on aerodynamic characteristics and flow structure over airfoils have been investigated in literature. Ma et al. [58] studied the effects of relative thickness on aerodynamic characteristics of airfoil at a low Reynolds number, and reported almost identical lift curves for the examined airfoils with different thicknesses. However, the thicker airfoils exhibited larger drag coefficient at low angle of attacks. Figure 2.9 represents the corresponding lift and drag coefficients. Sharma and Visbal [59] conducted a numerical investigation over the effect of airfoil thickness on onset of dynamic stall. Figure 2.10 demonstrates the sectional lift and drag coefficients at different angle of attacks as the airfoils are under a constant-rate pitch-up maneuver. The largest lift coefficient was observed for the thinnest airfoil, but the stall angle of attack was higher for the thicker airfoil. However, the thinnest airfoil had higher drag coefficients until its stall angle of attack. Ran et al. [60] studied the effect of relative thickness on the 
dynamic and aerodynamic characteristics of pitching airfoils, and reported that the higher relative thicknesses have larger dynamic lift-to-drag ratios. However, flow over delta wings have its own complications due to the presence of leading edge vortices and the interaction of shear layer with the vortical structure which prevent from driving a comprehensive conclusion about thickness effect over delta wings by using the results of thickness effect on airfoils.

Few studies have been devoted to investigate the thickness-to-chord ratio effect both on flow structure and aerodynamics of delta wings. Considering the effect of wing thickness on flow topology and aerodynamic forces of slender delta wings, the pioneering studies in this regard date back to 1960's, where Witcofski and Marcum Jr. [61] studied the effect of thickness and sweep angle on the longitudinal aerodynamic forces of a series of delta wings with sweep angles ranging from 45 to 90 degrees and thickness-to-chord $t / C$ ratios up to $30 \%$. They concluded that as the $t / C$ ratio increases, the slope of the lift curve and the angle of attack of the maximum lift-to-drag ratio increase, as shown in Figures 2.11 and 2.12 respectively. However, Figure 2.13 demonstrates that as the $t / C$ ratio increases, maximum lift-to-drag ratio decreases. Polhamus [18] stated that as delta wing thickness decreases the maintenance of attached flow become more difficult and many problems in performance, stability, and control arise. Ruo et al. [62] demonstrated the significance of wing thickness effect on unsteady transonic aerodynamics of oscillating delta wings using an analytical approach, and showed that as $t / C$ ratio increases the local Mach number over the wing decreases significantly. Lowson and Ponton [63] investigated asymmetry in vortex flows on conical bodies, where they reported reduction in flow asymmetry for thinner wings with 70 and 80 degrees sweep angles. Shih and Ding [37] explored the trailing edge jet control of leading edge vortices on a 60 deg swept delta wing with $4.2 \%$ and $20 \% t / C$ ratios, and they reported that an increase in the $t / C$ ratio moves the vortex breakdown location toward the apex of the wing. Parker [64] reviewed the aerodynamic characteristics of slender delta wings with sharp leading edges, and 
suggested the increase of thickness as one of the reasons that moves the vortex system outboard. Lowson and Riley [65] and Thompson [66] reported movement of vortex breakdown location toward apex as the wing thickness increases in slender delta wings. Figure 2.14 demonstrates vortex breakdown location versus angle of attack for three different delta wings, where two of them have identical bevel shape but different $t / C$ ratios [66]. Saltzman and Ayers [67] reviewed flight-to-windtunnel drag correlation. Figure 2.15 represents drag versus Mach number for two airplanes with the same sweep angle of $60 \mathrm{deg}$ and different $t / C$ ratios, where they concluded that thicker wing experiences drag divergence in lower Mach numbers and with larger wave drag increments.

For non-slender delta wings, a very few studies have addressed the thickness effect on aerodynamic forces and flow structure. The aeroelastic behavior of a cropped delta wing with a variable position store and 45 deg sweep angle was investigated by Golparvar et al. [68]. They have examined the effect of wing thickness on flutter speed and frequency of the wing for different spanwise location of the store as demonstrated in Figure 2.16, and concluded that with an increase in $t / C$ ratio flutter speed increases, however flutter frequency decreases with increase in $t / C$ ratio up to the turning point of the store span location. McClain et al. [69] investigated the unsteady aerodynamics of free-to-roll non-slender delta wings using series of 50 deg swept delta wing models, including two models with semicircular leading edges and thickness-to-chord ratios of $t / C=4 \%$ and $10 \%$. The lift force measurement results indicated almost identical $C_{\text {Lmax }}$ and stall angle of attack for these two wing models. Although, the amplitude of roll oscillations in small incidences decreased with decreasing wing $t / C$ ratio. Wang and $\mathrm{Lu}$ [57] primarily investigated the effect of leading edge bevel angle on the aerodynamic performance of a $50 \mathrm{deg}$ swept delta wing using wings with thickness-to-chord ratios of $t / C=2 \%, 6.7 \%$, and $10 \%$. They concluded for all of the wings with different bevel angles that as the $t / C$ ratio increases, drag force increases and the lift-to-drag ratio decreases. Kawazoe et al. [9] determined the flow structure and aerodynamic forces of a 45 
deg swept delta wing with leading edges in half circle shape for the thickness-tochord ratios of $t / C=2.7 \%$ and $9.1 \%$, in which the thicker wing exhibited lower maximum lift and lift-to-drag ratio, and higher drag and stall angle as represented in Figures 2.17 and 2.18. In addition, as demonstrated in Figure 2.19, they reported that the vortex of thinner wing reaches the centerline earlier, indicating the appearance of vortex breakdown and three-dimensional separation in lower angle of attack for the thinner wing. Recently, Gülsaçan et. al. [8] investigated the effect of $t / C$ ratio on flow structure of a $35 \mathrm{deg}$ swept delta wing with four $t / C$ ratios varying from $4.75 \%$ to $19 \%$ using pressure measurement, laser illuminated smoke visualization, and particle image velocimetry. They have reported that as the wing thickness increases, the flow structure transforms from leading edge vortex to threedimensional separated flow regime. In addition, for the low angles of attack where all wings experience leading edge vortex structure, the strength of the vortex structure increases as the $t / C$ ratio increases. However, three-dimensional separated region appears in lower angle of attack for the thicker wing. Figure 2.20 represents a comparison of patterns of time-averaged streamlines at attack angle of $10 \mathrm{deg}$, where the thicker wing experiences swirl flow as an indication of three dimensional separation. 


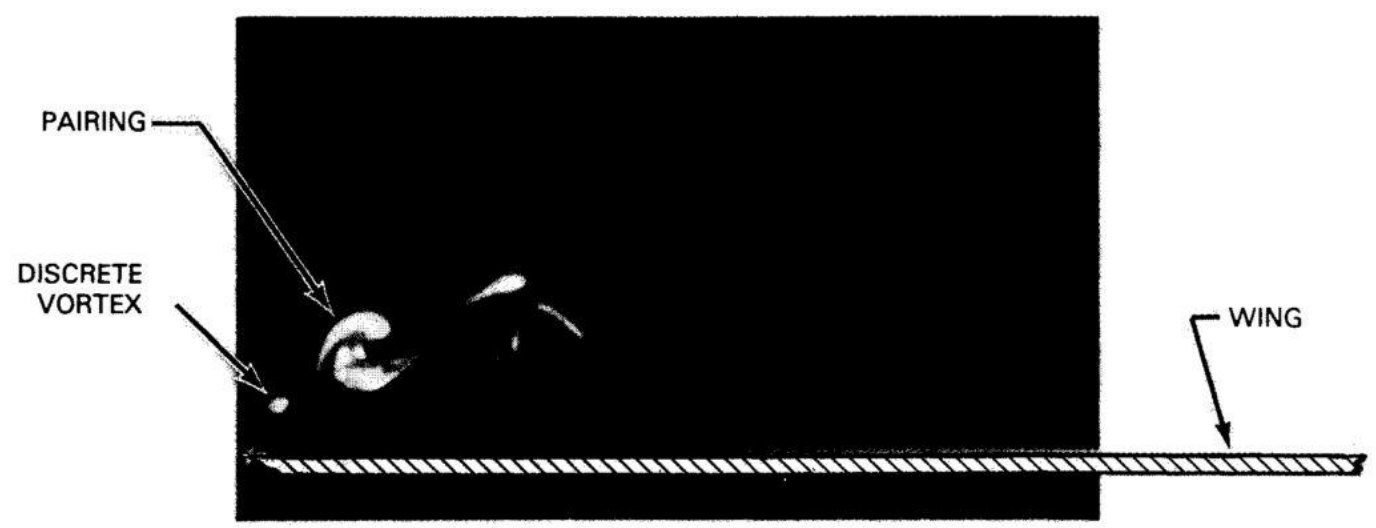

Figure 2.1 illustration of discerete vortices emanating from the leading edge [16].

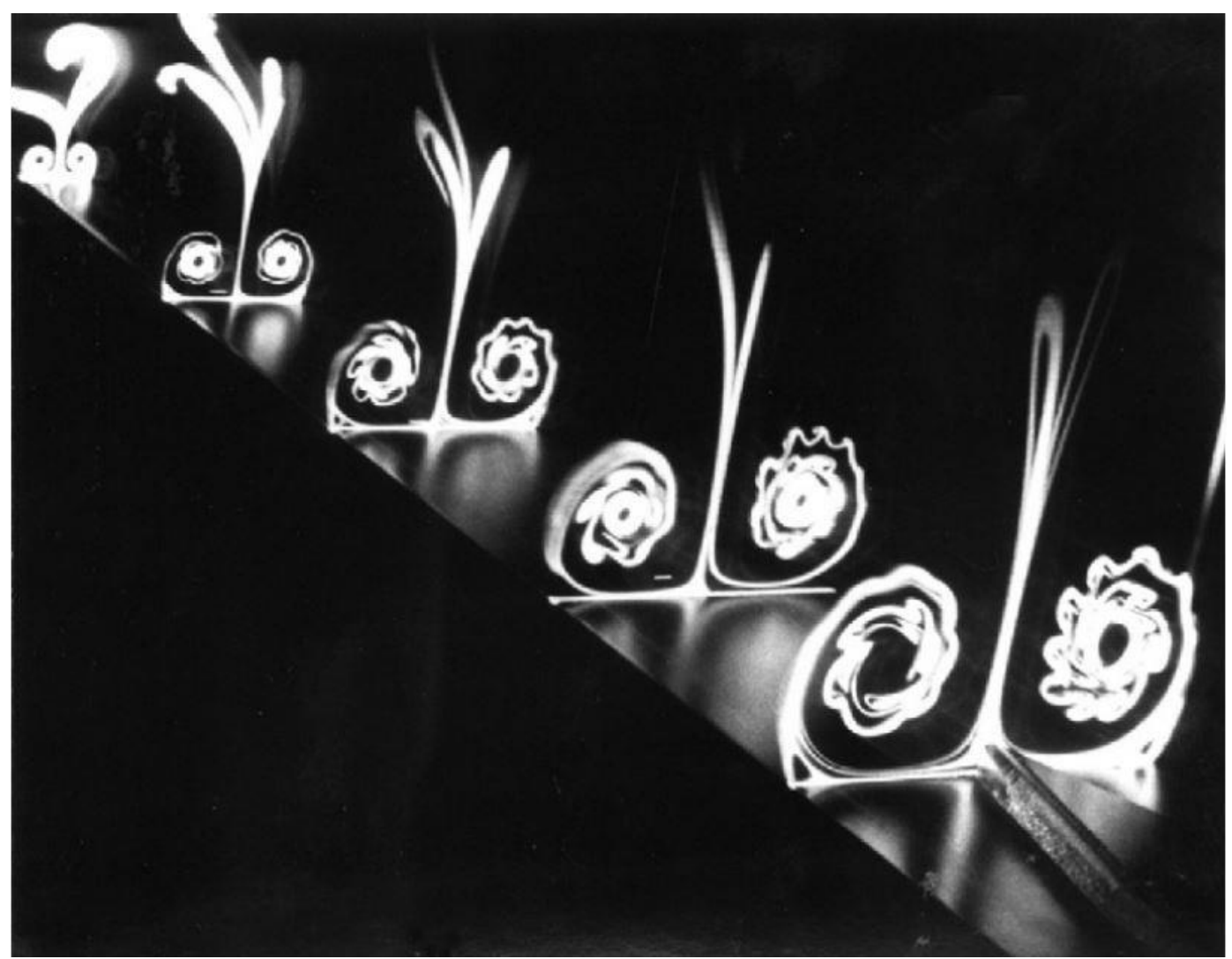

Figure 2.2 Laser light sheet illumination of primary vortices and the discrete vortices that are feeding them [17]. 


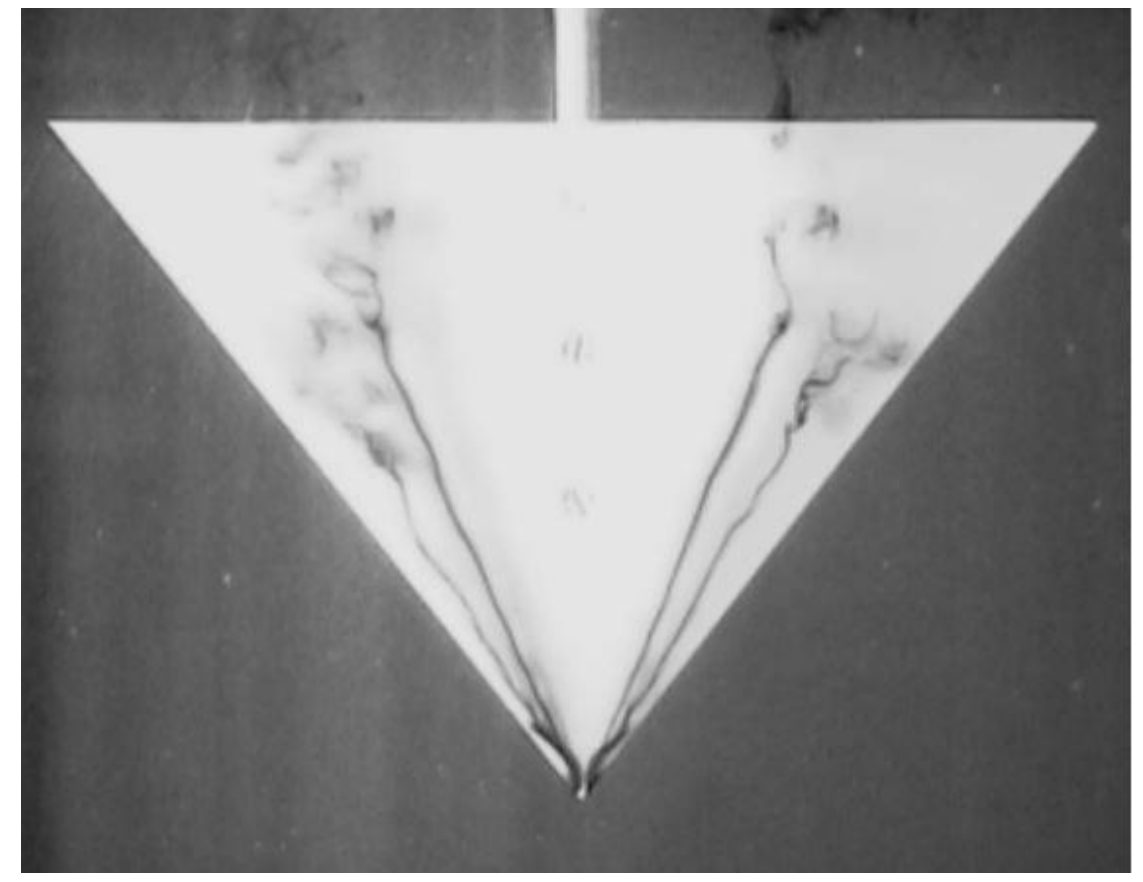

Figure 2.3 Flow visualization of dual vortex structure [21].
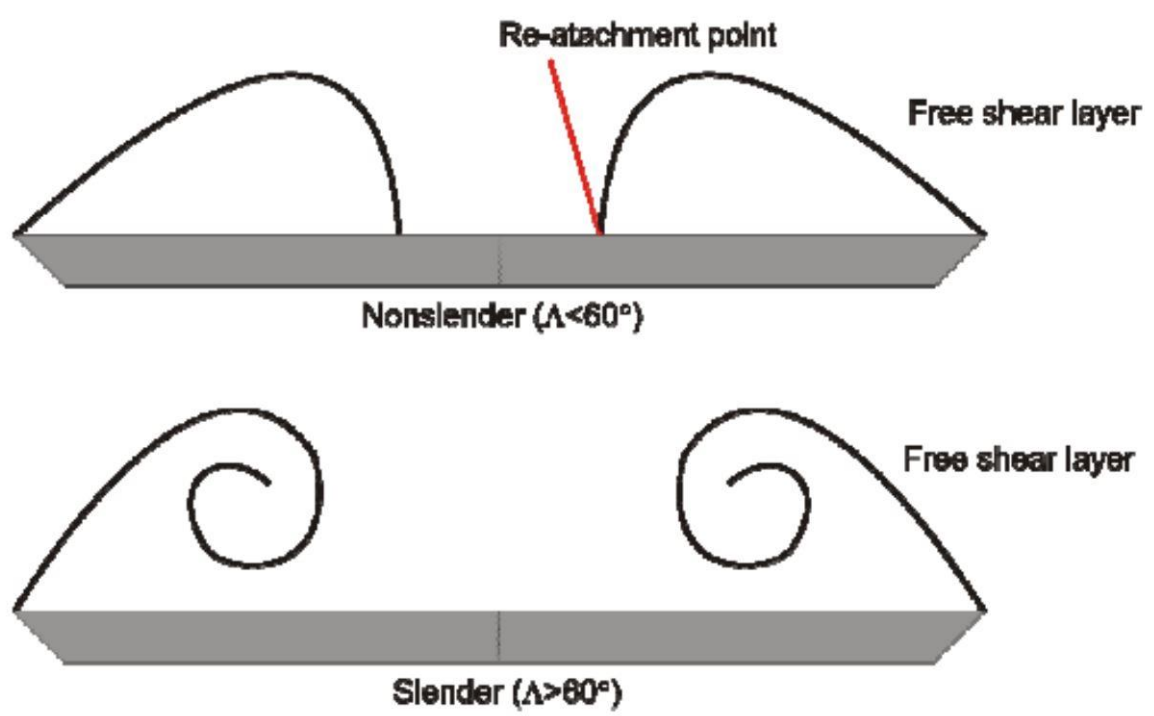

Figure 2.4 Schematic of vortical flow for non-slender delta wing with the presence of vortex Reattachment (top) and slender delta wing without the reattachment (bottom) [21]. 


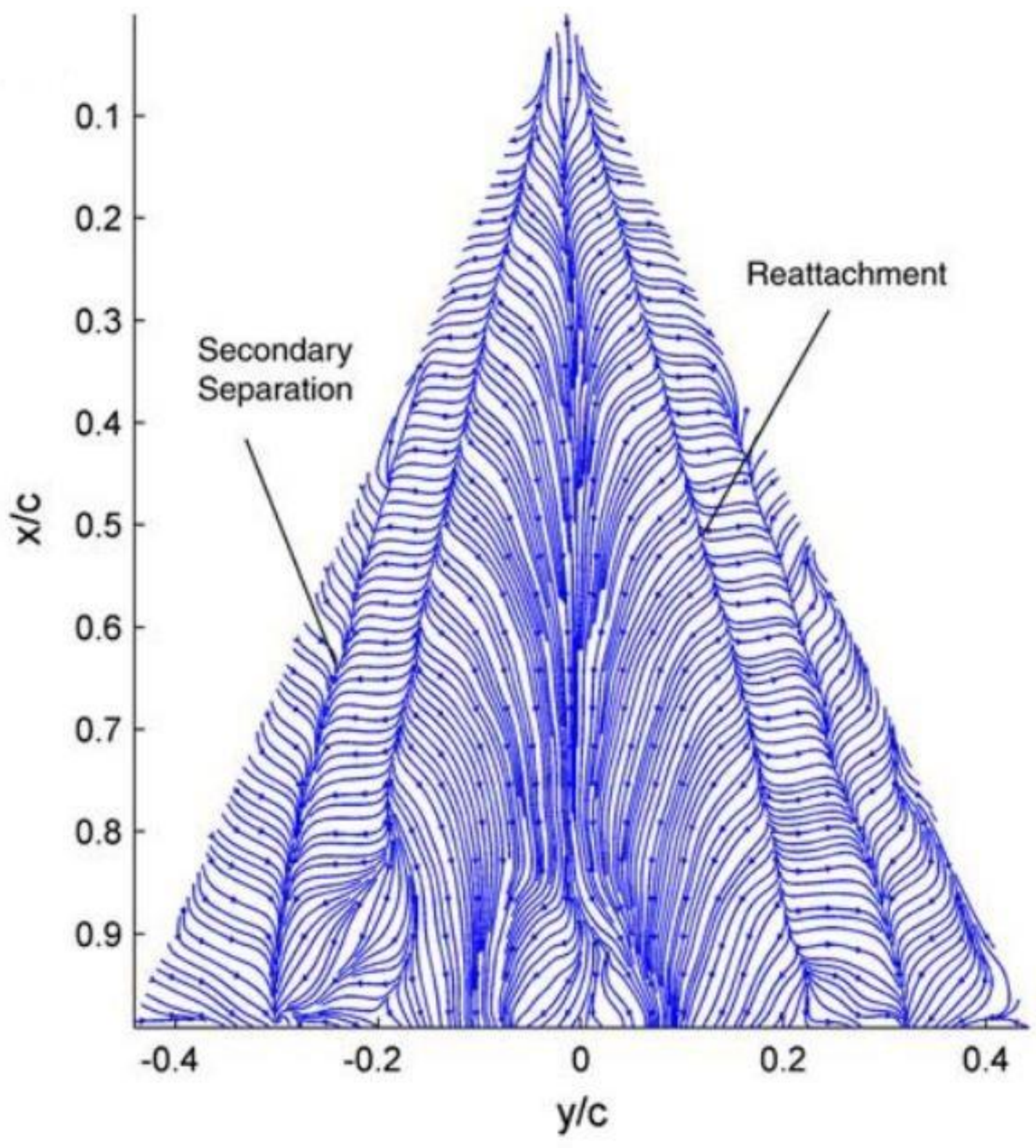

Figure 2.5 Skin friction lines and the corrosponding secondary separation and reattachment lines [22]. 

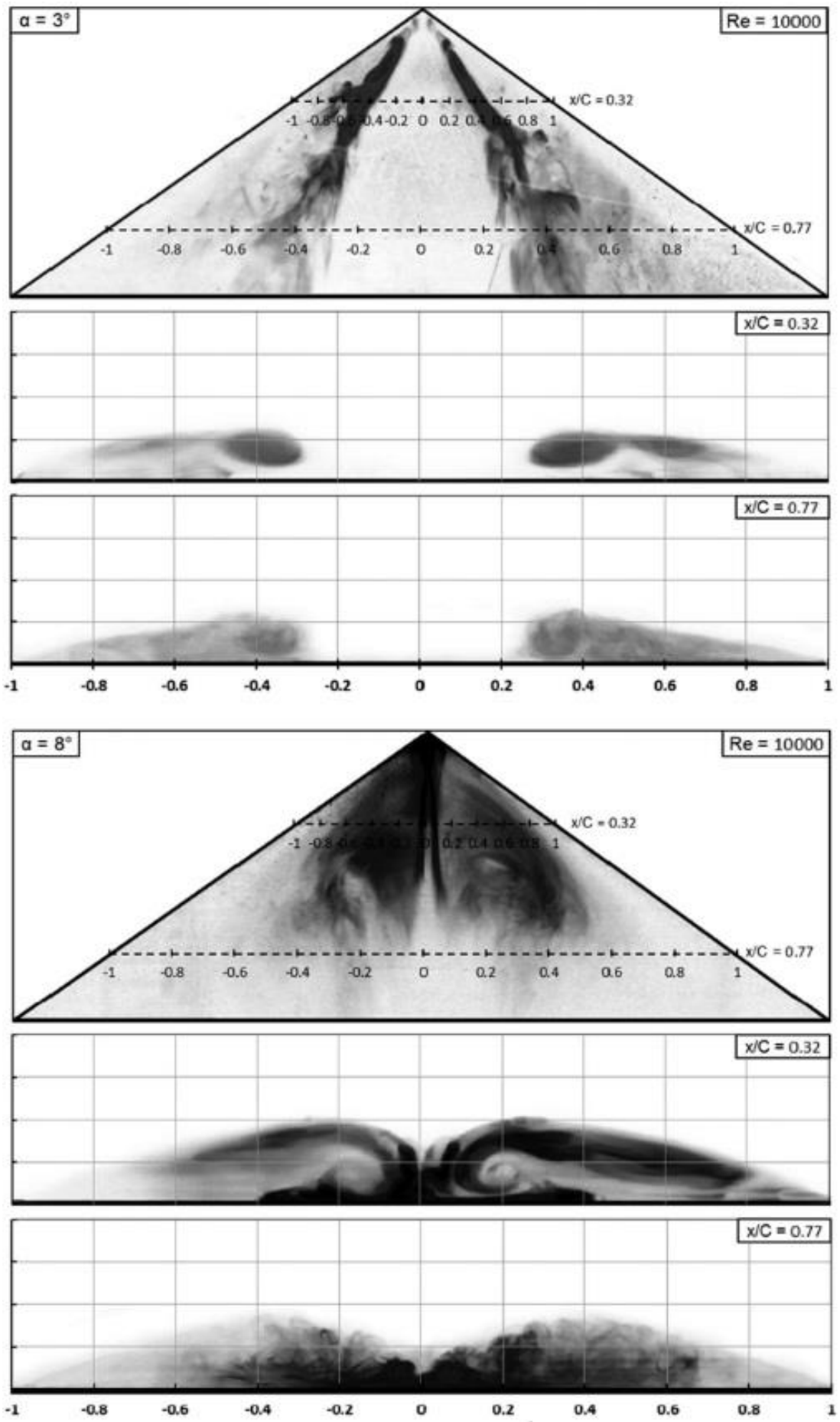

Figure 2.6 Smoke visualization results at $R e=1 \times 10^{4}$ for $\alpha=3^{\circ}$ and $8^{\circ}$ for 45 deg swept delta wing [23]. 


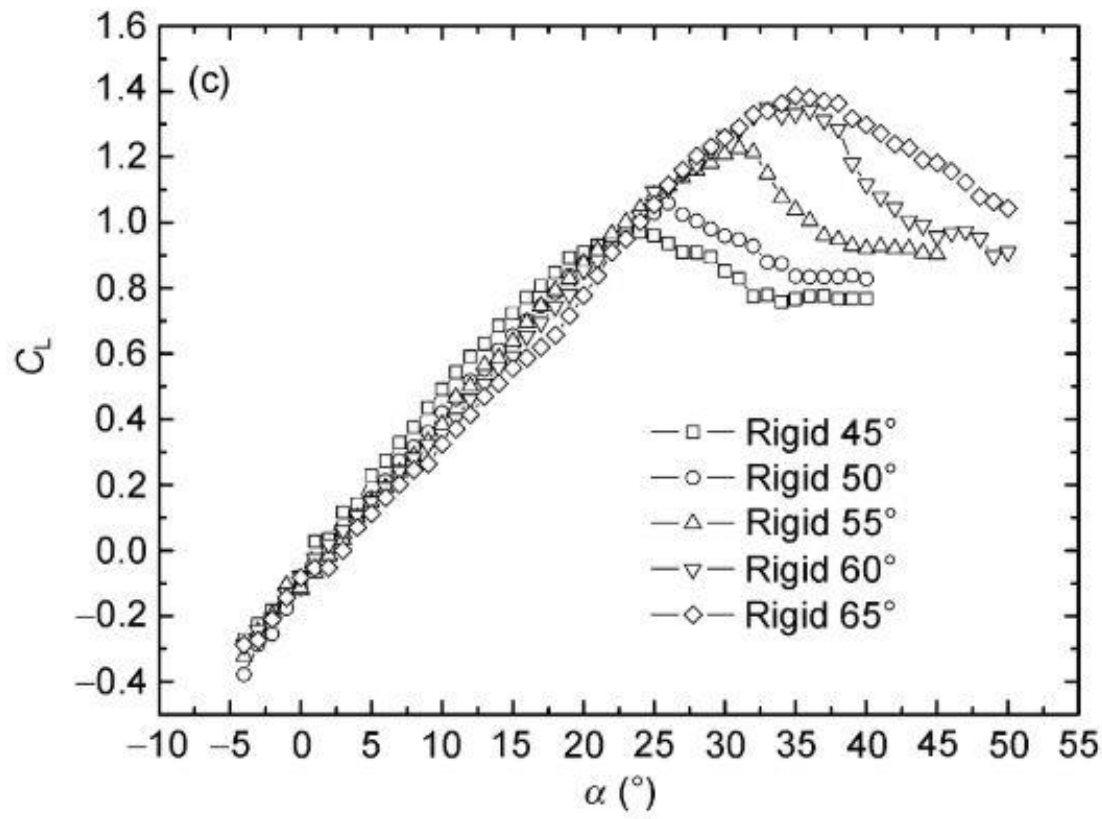

Figure 2.7 $C_{L}$ versus $\alpha$ for rigid wings with the change of sweep angle [51].

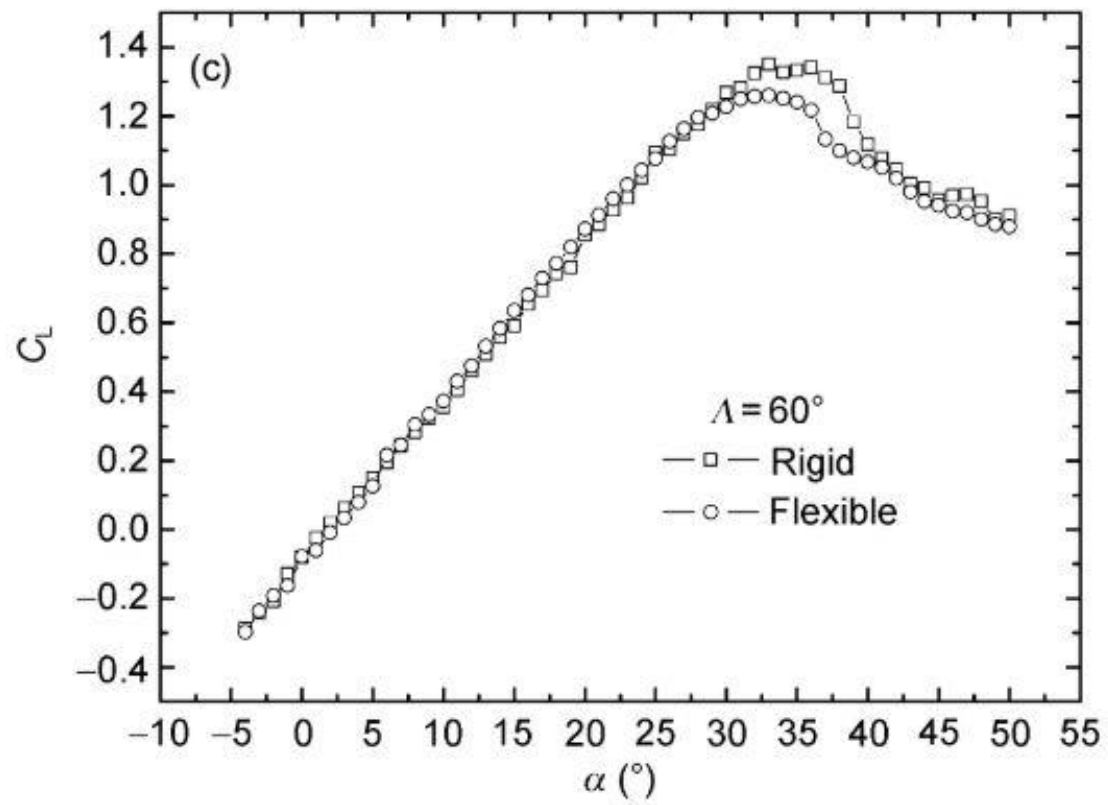

Figure 2.8 Comparison of $C_{L}$ for rigid and flexible wings with 60 deg sweep angle [51]. 


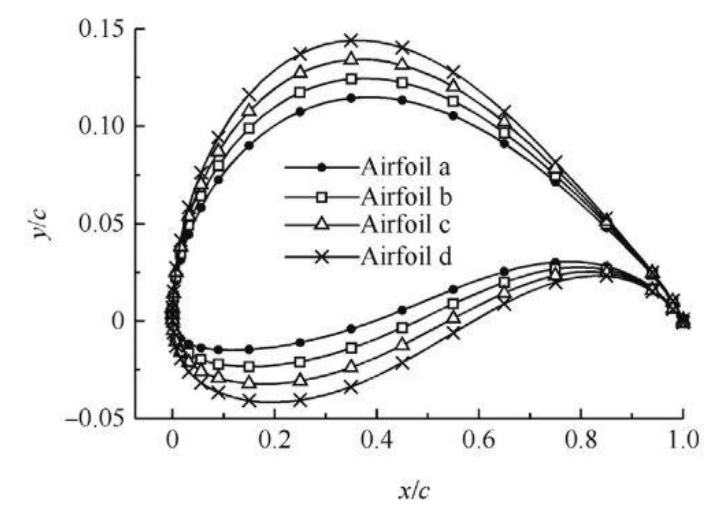

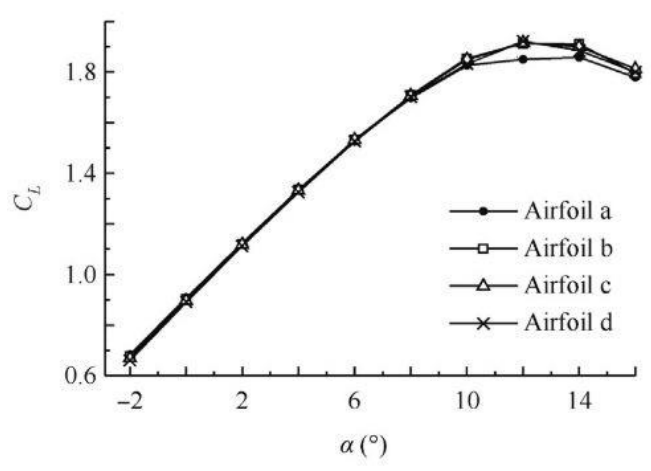

(a) Lift coefficients

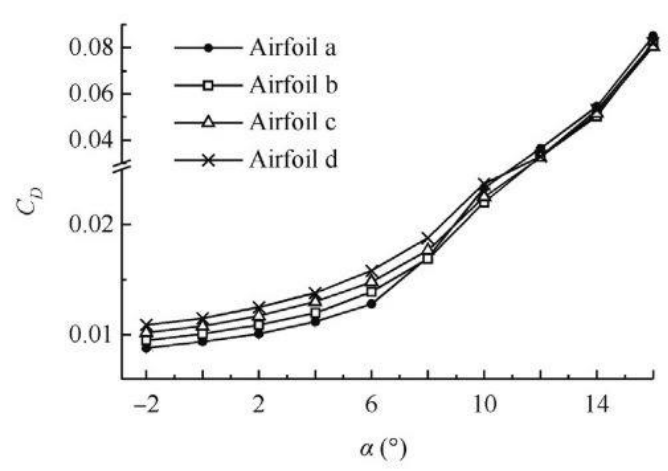

(b) Drag coefficients

Figure 2.9 Shape of airfoils with different relative thickness values (top), and corrosponding (a) lift and (b) drag coefficient values (bottom) [58]. 


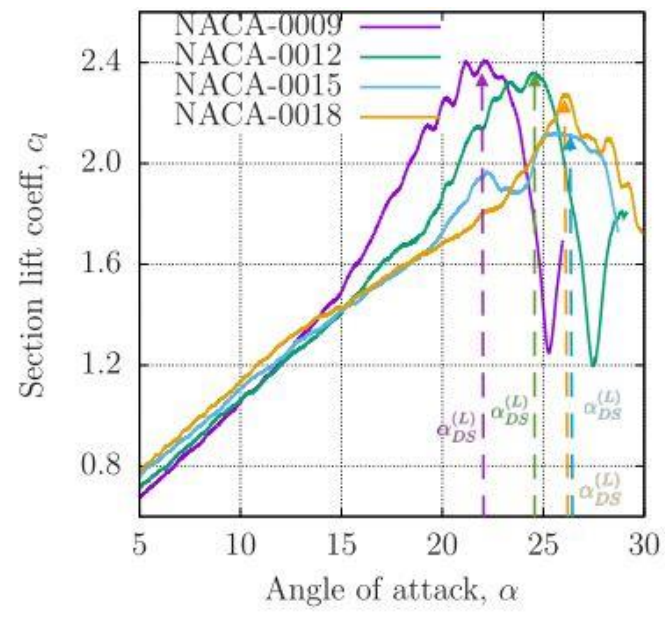

(a) $c_{l}$

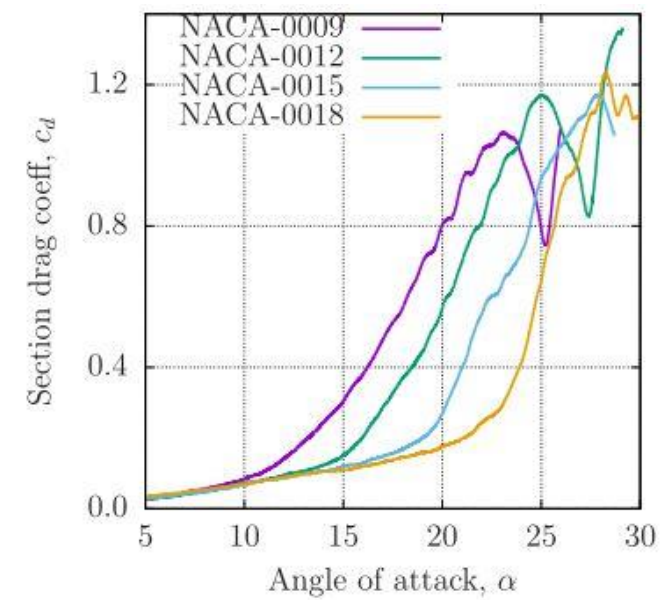

(b) $c_{d}$

Figure 2.10 Sectional lift and drag coefficients versus angle of attack during a constant pitch rate maneuver for airfoils with thickness ratios of $t / C=0.09$, $0.12,0.15$, and 0.18 [59].

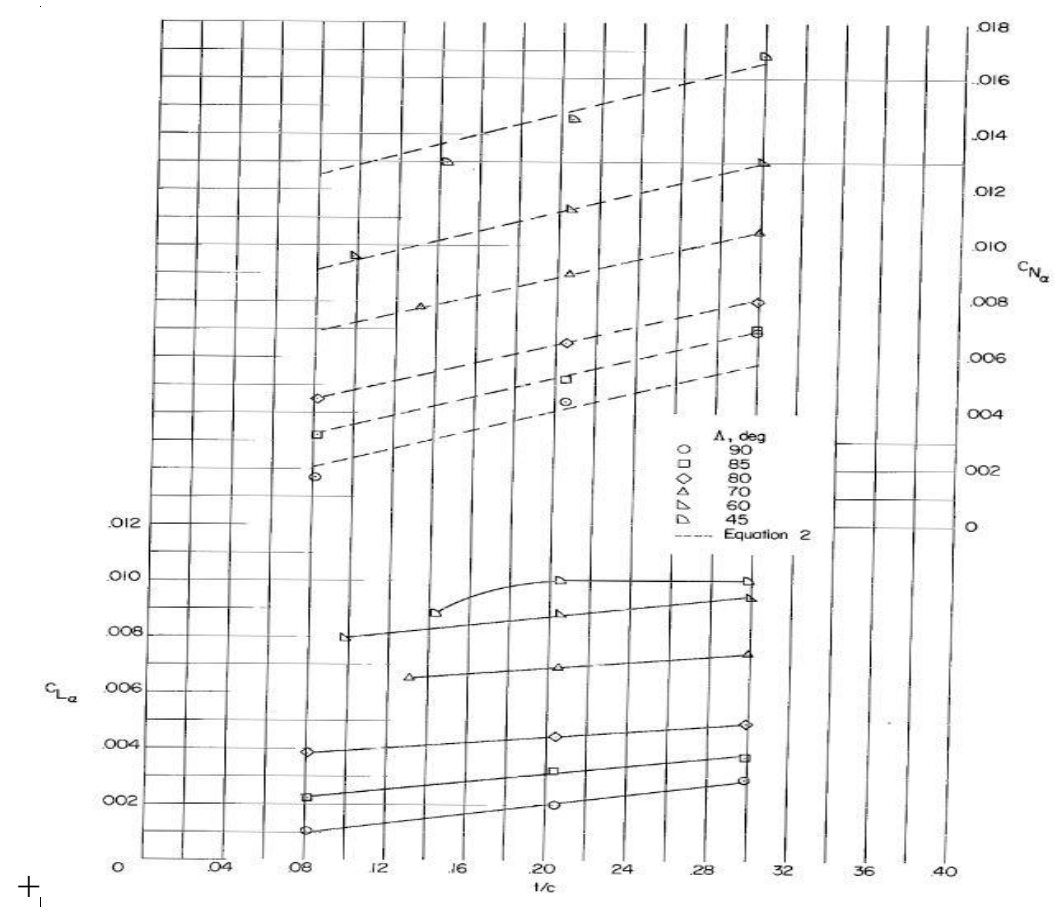

Figure 2.11 Variation of lift and normal force coefficients slopes versus $t / C$ ratio [61]. 


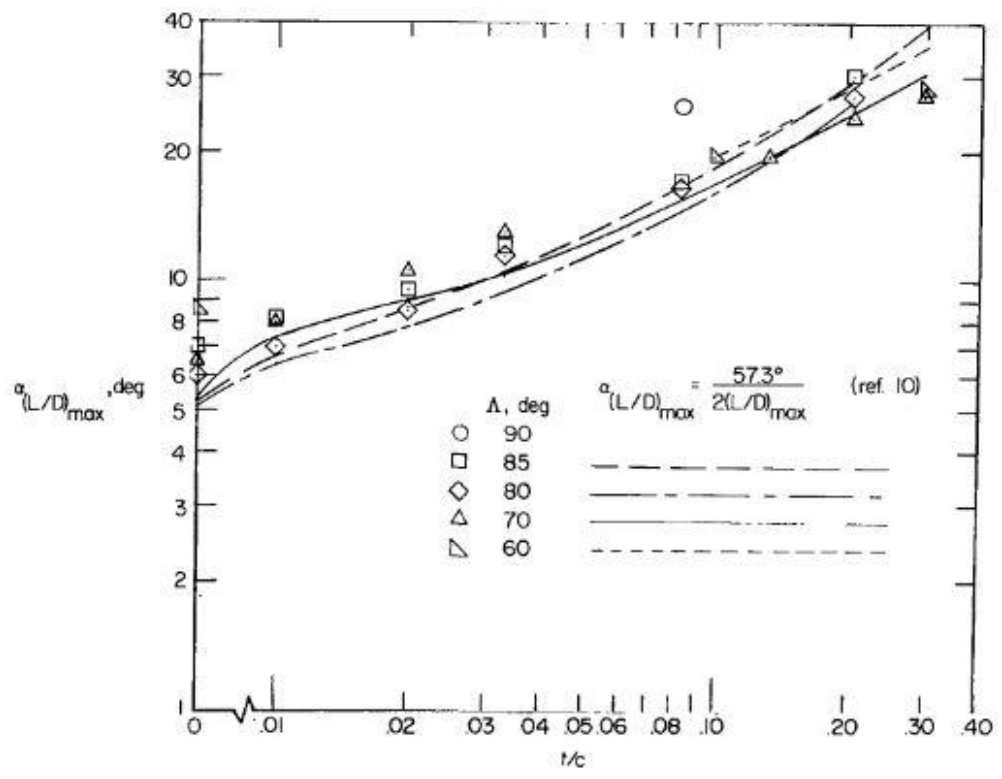

Figure 2.12 Angle of attack of maximum lift-to-drag ratio versus $t / C$ ratio [61].

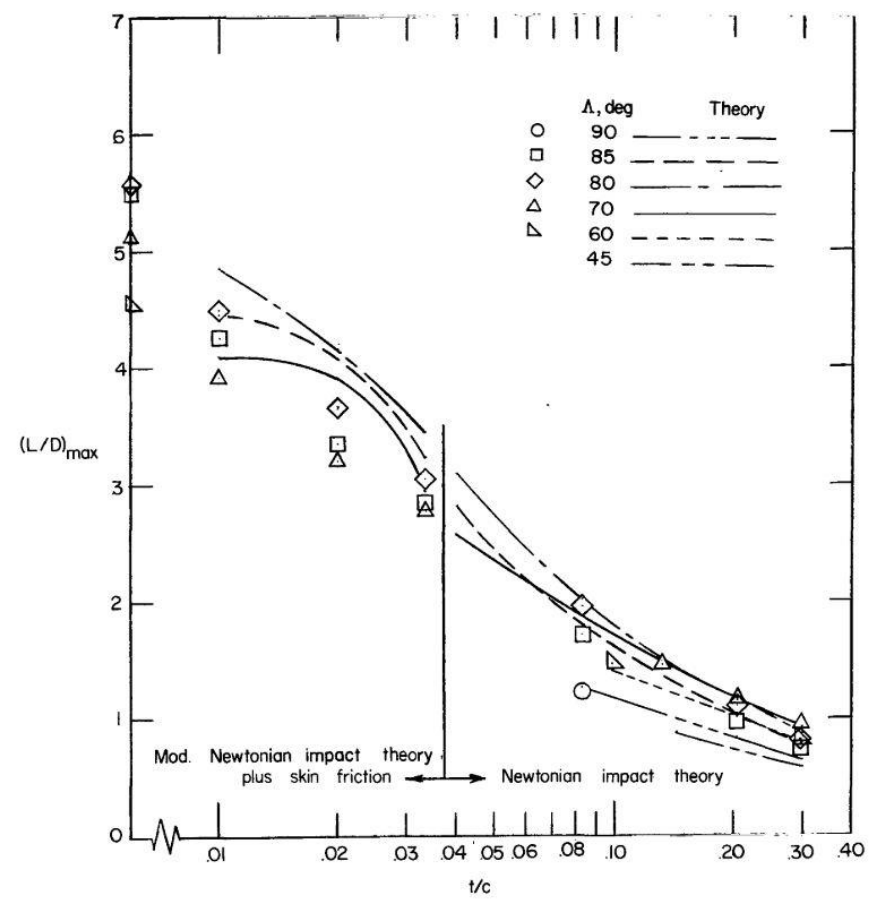

Figure 2.13 Variation of maximum lift-to-drag ratio versus $t / C$ ratio for different sweep angles [61]. 


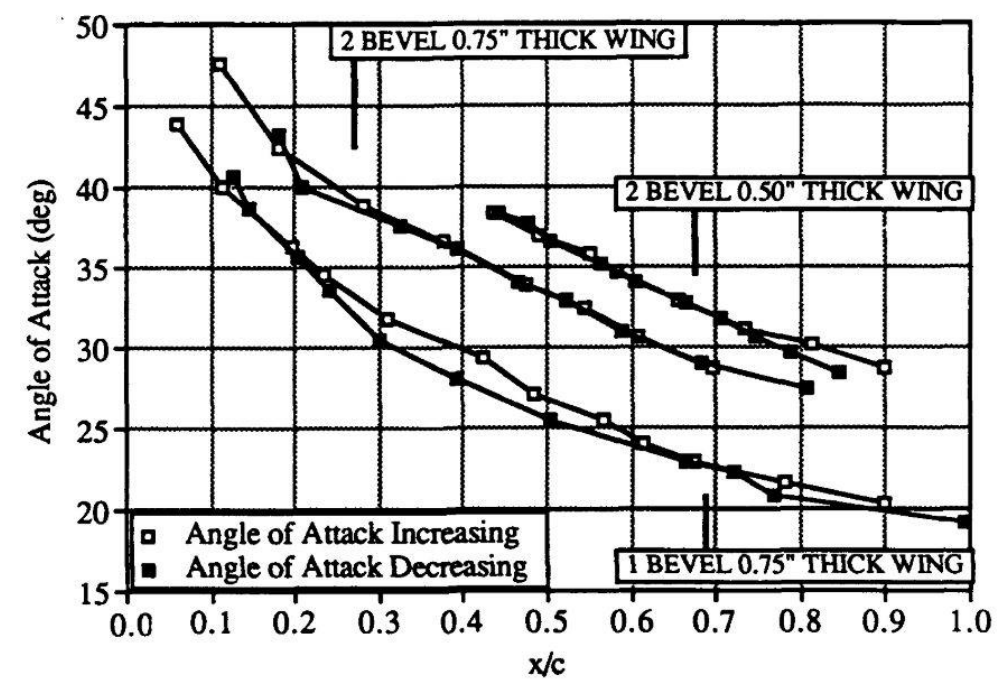

Figure 2.14 Thickness and leading edge geometry effects on primary vortex breakdown location [66].

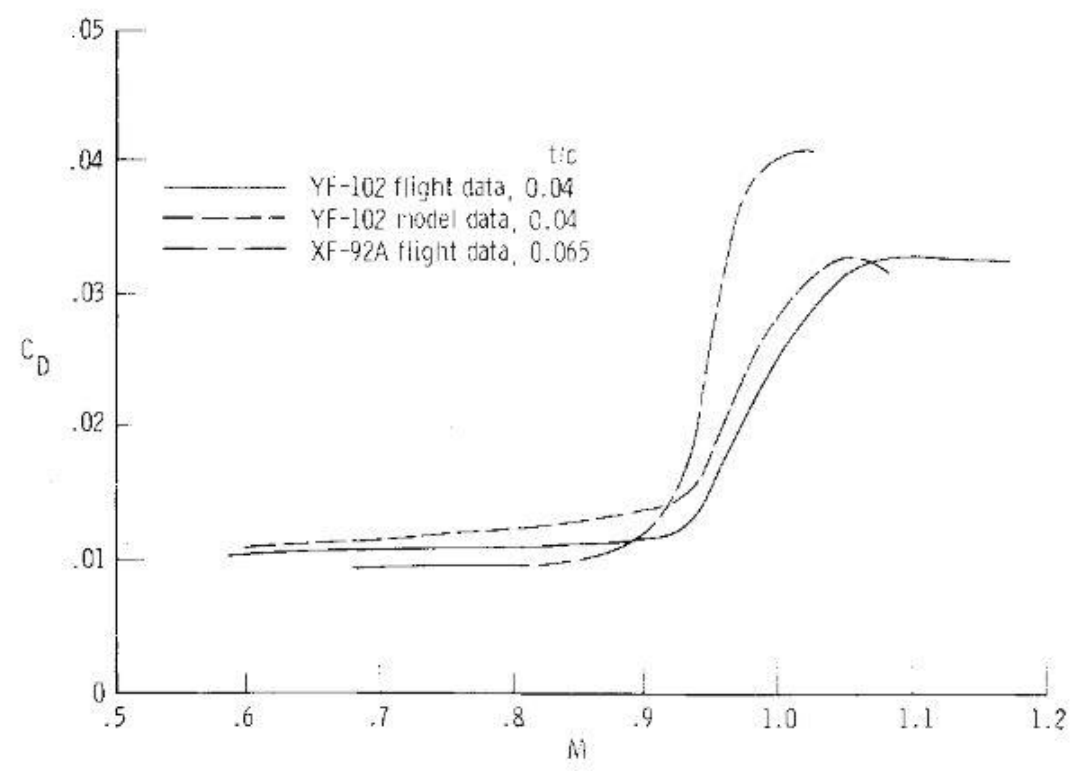

Figure 2.15 Effect of wing thickness-to-chord ratio on drag divergence, Mach number, and the wave drag increment [67]. 


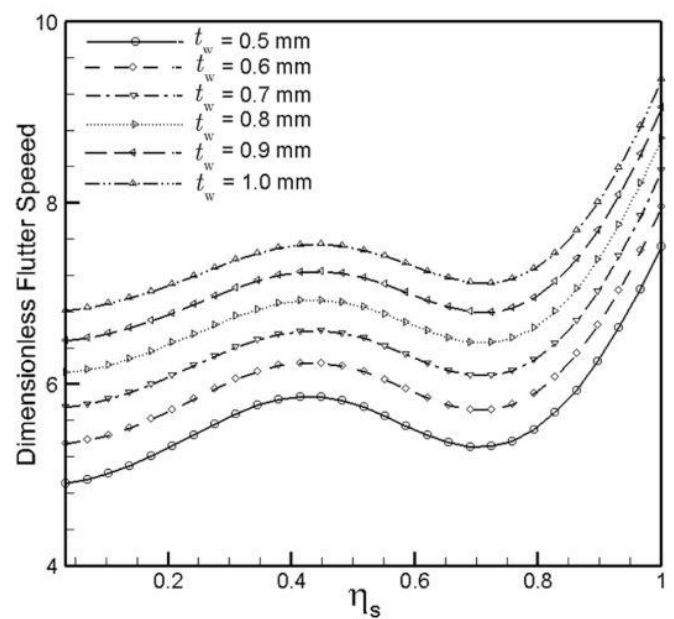

(a)

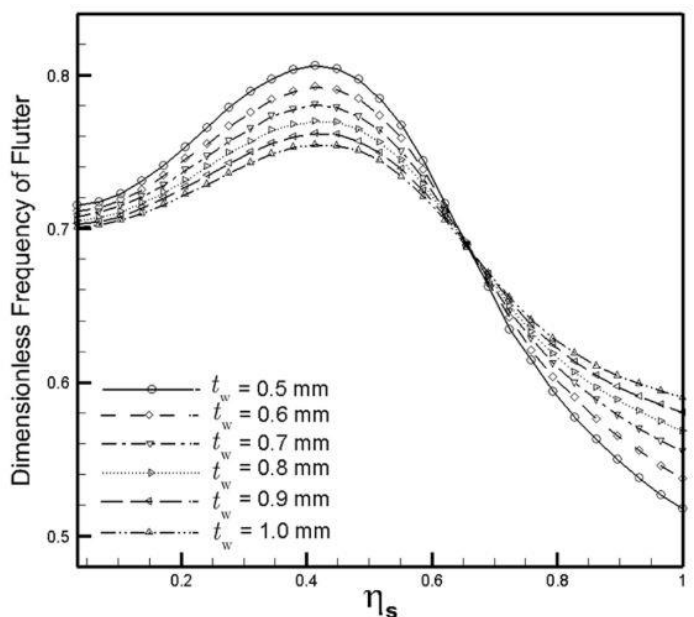

(b)

Figure 2.16 Wing thickness effect on (a) dimensionless flutter speed and (b) dimensionless flutter frequency, versus dimensionless span location of store [68].

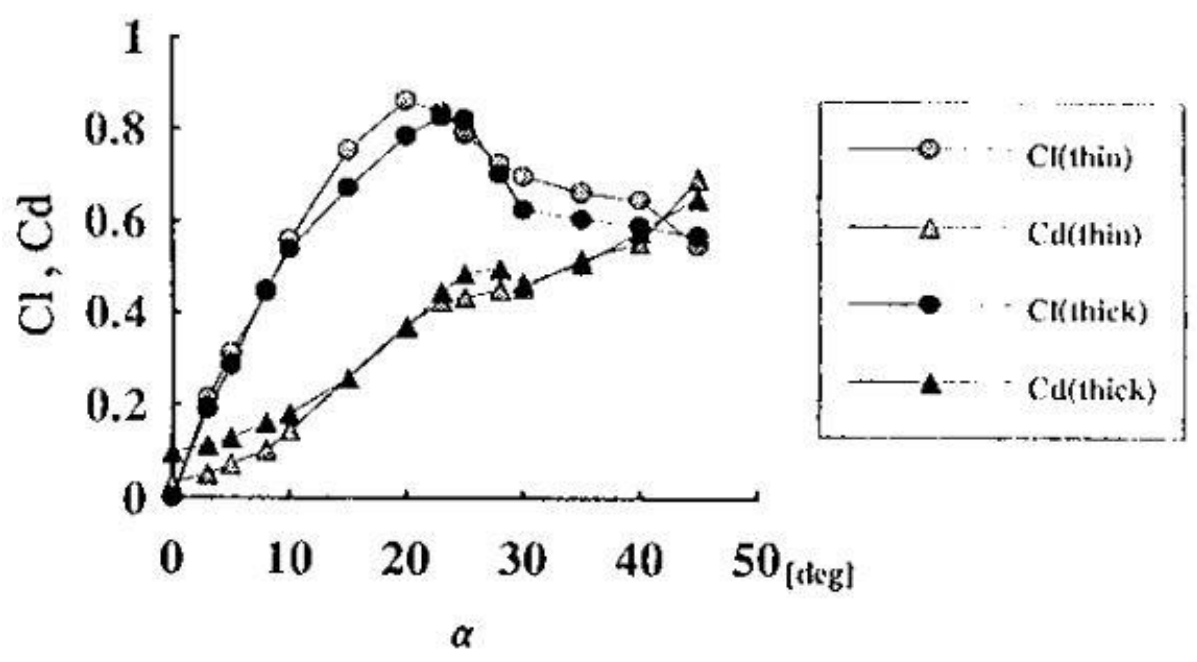

Figure 2.17 Wing thickness effect on $C_{L}$ and $C_{D}$ values [9]. 


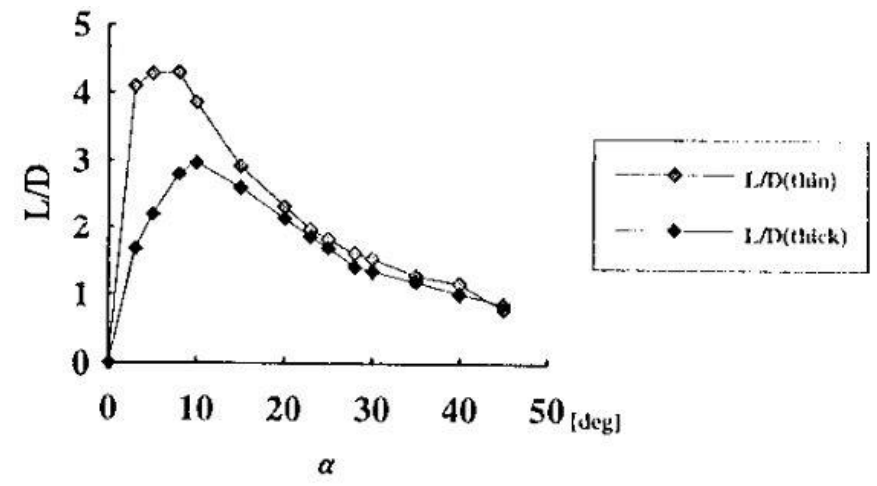

Figure 2.18 Lift-to-drag ratio versus angle of attack for thick and thin wing models [9].

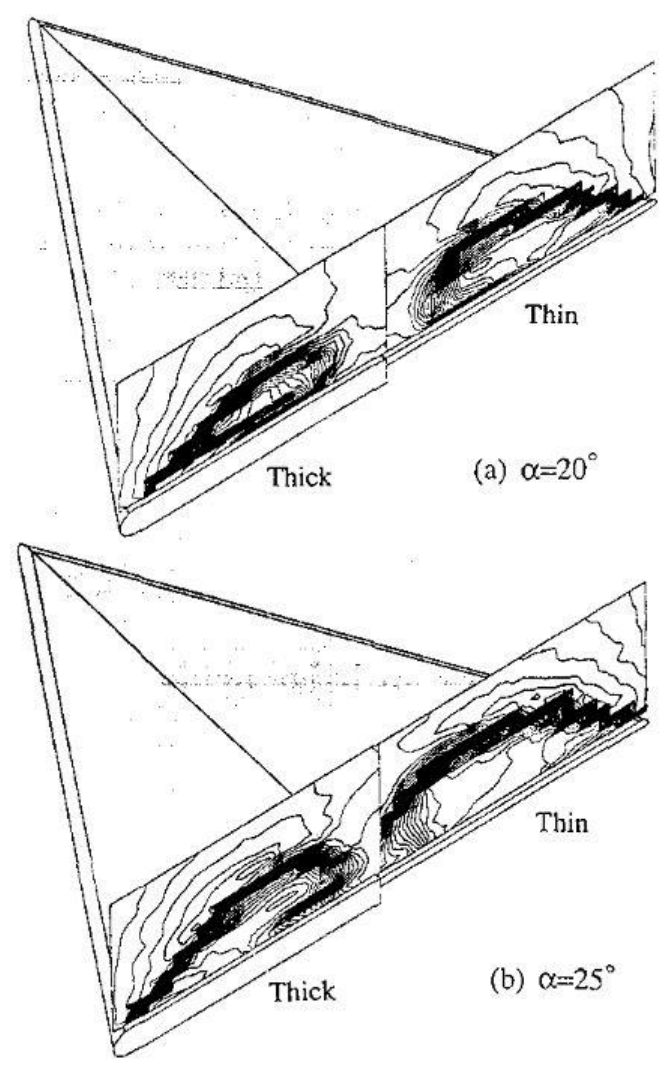

Figure 2.19 Difference between thin and thick wing in velocity contours at (a) $\alpha=20^{\circ}$ (b) $\alpha=25^{\circ}$ [9]. 


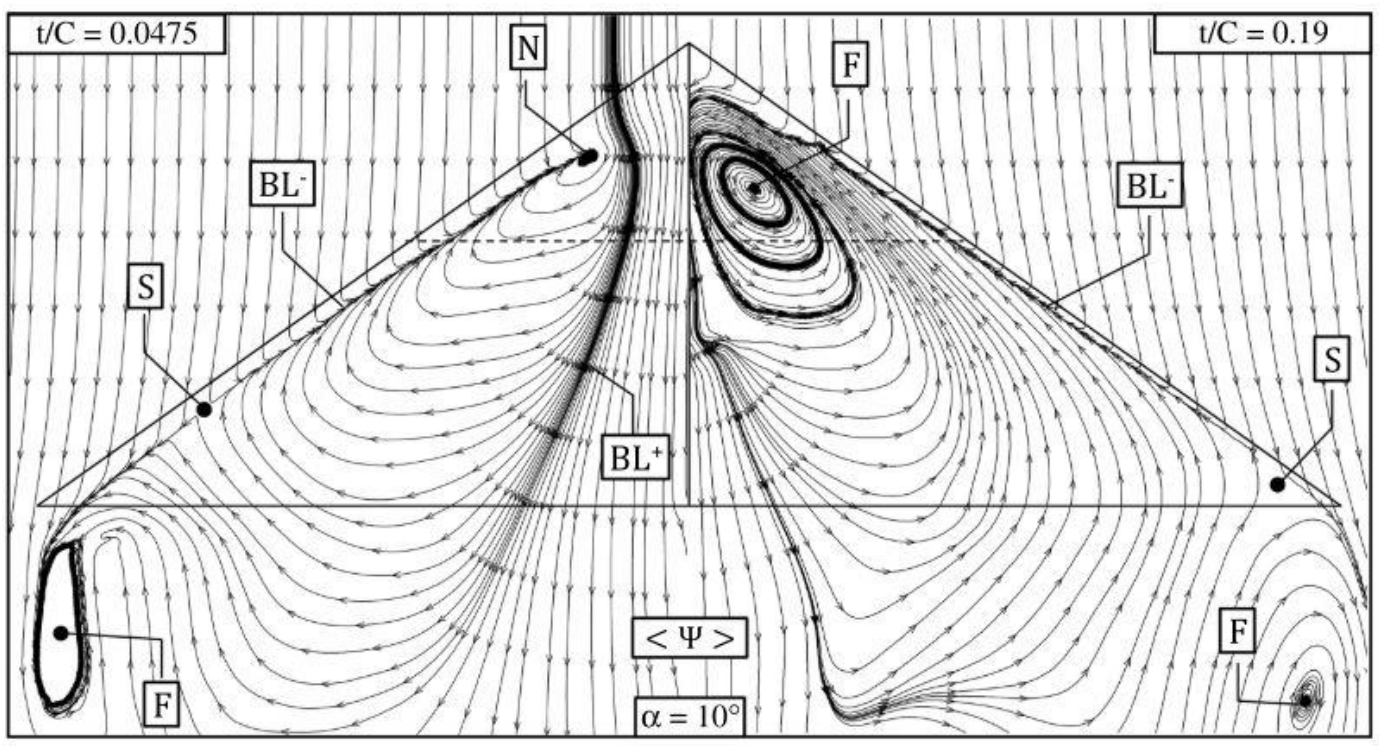

Figure 2.20 Effect of $t / C$ ratio on patterns of time-averaged streamlines [8]. 


\section{CHAPTER 3}

\section{EXPERIMENTAL SET-UP AND MEASUREMENT TECHNIQUES}

This chapter summarizes the general description of utilized experimental facilities and techniques together with the corresponding experimental matrix for each measurement technique.

\subsection{Wind Tunnel}

Experiments were performed in a low-speed, suction type, and open-circuit wind tunnel, located in the Fluid Mechanics Laboratory of the Mechanical Engineering Department at the Middle East Technical University. As Figure 3.1 represents, the air comes via two intakes into the settling chamber. In order to prevent intrusion of any unfavorable material and enhance the air uniformity, fine mesh screens are placed at the inlets. Then, air speeds up in the contraction cone with a contraction ratio of 8:1and inters the test section, which is $750 \mathrm{~mm}$ wide, $510 \mathrm{~mm}$ deep, and $2000 \mathrm{~mm}$ long and made of plexiglas to provide fully transparent view of flow field for the optical experimental techniques. After the test section there is a diffusion chamber followed by a fan, which provides the suction power for running the tunnel. The maximum speed of the wind tunnel is $30 \mathrm{~m} / \mathrm{s}$.

Experiments were conducted at Reynolds numbers of $R e=1.5 \times 10^{4}, 3.5 \times 10^{4}$, and $1 \times 10^{5}$ based on the wing root chord $C$, and the corresponding free stream velocities were $U_{\infty}=1.5,3.5$, and $10 \mathrm{~m} / \mathrm{s}$, respectively. Reynolds number is given by Equation 3.1, where $C$ is the chord length and $v$ is the kinematic viscosity of air at free stream temperature. For this range of free stream velocities, the measured turbulence intensities in the tunnel test section were less than $1 \%$. The maximum blockage ratio at the highest attack angle of $\alpha=30 \mathrm{deg}$, was less than $3 \%$. 


$$
R e=\frac{U C}{v}
$$

\subsubsection{Wind Tunnel Characterization}

In order to obtain the required fan powers for desired velocities, wind tunnel characterization was performed prior to the experiments using Laser Doppler Anemometry (LDA), which were verified by Pitot-static tube measurements. The data were obtained at a fixed point inside the test section, and different environmental conditions such as temperature, humidity, and local atmospheric pressure were considered in the calibration. Figure 3.2 shows the required fan power along with turbulence intensity with respect to velocity magnitudes. As indicated in Figure 3.2, the maximum turbulence intensity was $0.9 \%$.

\subsection{Flow Measurement Techniques}

\subsubsection{Laser-Illuminated Flow Visualization}

The qualitative investigation of flow structure was performed using laser illuminated smoke visualization, where vaporized liquid paraffin was generated via a commercial smoke generator and pressurized by $\mathrm{CO}_{2}$ gas coming from pressure tank with a check valve to control the flow rate. The smoke was injected through the smoke holes on the surface of the wing, and illuminated via a diode-pumped solid-state green laser with $532 \mathrm{~nm}$ wavelength and $400 \mathrm{~mW}$ power output. The laser sheet was positioned parallel to vortex trace as shown in Figure 3.3. The images were captured using a digital single-lens reflex camera, which was positioned outside the test section and set at the angle of attack of the wing. The images were then converted to black-and-white using Adobe Photoshop.

\subsubsection{Surface Pressure Measurement}

Pressure measurements were obtained at a chordwise distance of $x / C=0.5$ using 16-channel pressure scanner, which had 16 silicon piezo-resistive pressure sensors with a range of $0-2.5 \mathrm{kPa}$. Figure 3.4 demonstrates the location of the pressure measurement plane over the wing. Preliminary experiments were carried out to 
ensure the symmetry on spanwise pressure distribution, and afterward only the half of span measurements were performed. Pressure data were accumulated for $30 \mathrm{~s}$ at $500 \mathrm{~Hz}$ frequency, and then the dimensionless pressure coefficient $C_{p}$ was calculated using the following equation:

$$
C_{p}=\frac{\bar{p}-p_{\infty}}{\frac{1}{2} \rho U^{2}}=\frac{\bar{p}-p_{\infty}}{p_{d y n}}
$$

\subsubsection{Particle Image Velocimetry (PIV) Measurements}

High-image-density Particle Image Velocimetry (PIV) technique was utilized to extract quantitative cross-flow pattern at a chordwise distance of $x / C=0.5$ as demonstrated in Figure 3.4. TSI PIV system using a dual-pulsed Litron Nd:YAG laser with laser pulse pairs up to $200 \mathrm{~mJ}$ and maximum repetition rate of $15 \mathrm{~Hz}$, was used. The laser pulses and the camera were synchronized using a LaserPulse Synchronizer. The images of the illuminated tracer particles were taken via a digital Powerview $^{\mathrm{TM}}$ Plus, CMOS Camera which have $2048 \times 2048$ pixel resolution and equipped with a Nikon $50 \mathrm{~mm} F 1.8$ lens. The camera was positioned outside the tunnel and kept perpendicular to the vertical side of the test section. A mirror was placed downstream of the wing with an inclination of 45 deg to freestream and distance equal to seven chords from the wing. Preliminary tests were performed to assure that the upstream flow over the wing was uninfluenced by the mirror. The interrogation window size was as $16 \times 16$ pixels, which corresponds to the effective grid size of $\delta / C=0.019$. Sequences of 200 instantaneous velocity fields were used to evaluate the time-averaged velocity field $\langle V\rangle$ and the contours of constant time-averaged dimensionless vorticity $\langle\omega C / U\rangle$. Furthermore, in order to obtain velocity fluctuations, root mean square (rms) values of normal to surface component of velocity $w_{r m s} / U$ and transverse component of velocity $v_{r m s} / U$ of the flow field were calculated using Tecplot Focus software. Equation 3.3 shows 
the rms calculation of transverse component of velocity, $v_{r m s}$. The code that was used to calculate the rms values in Tecplot Focus is provided in Appendix A.

$$
v_{r m s}=\sqrt{\left\{\frac{1}{N} \sum_{n=1}^{N}\left(v_{n}(x, y)-\bar{v}(x, y)\right)^{2}\right\}}
$$

\subsubsection{Force Measurements}

For the characterization of aerodynamic performance, lift and drag force measurements were conducted using a custom design strut, which includes strain gauges mounted on horizontal and vertical parts of the strut to build three full Wheatstone bridges as demonstrated in Figure 3.5. The bridges were incorporated with the National Instruments SCC-SG Full-Bridge strain gauge modules and the calibration was performed to extract the lift and drag coefficients. Figure 3.6 represents the calibration curves of the all three full bridge strain gauges where M1, $\mathrm{M} 2$, and M3 represent the measured strains in full bridge 1, 2, and 3 respectively. The calibration curves demonstrate a linear trend of measured strains with respect to the applied forces. The following equations are applied to calculate the normal to surface, $N$, and the axial, $A$, aerodynamic forces using the measured strains from the full bridges.

$$
\begin{gathered}
N=\frac{M_{2}-M_{1}}{X L_{1}} \\
A=\frac{M_{3}-M_{2}-N L_{2}}{X L_{3}}
\end{gathered}
$$

In these equations $X$ is the calibration coefficient to convert the applied force to measured strains which was obtained from calibration. Then, the following 
equations are used to calculate the corresponding drag and lift coefficients, $C_{D}$ and $C_{L}$, respectively:

$$
\begin{aligned}
& C_{D}=\frac{N \sin \alpha+A \cos \alpha}{0.5 \rho U^{2} A_{R e f}} \\
& C_{L}=\frac{N \cos \alpha-A \sin \alpha}{0.5 \rho U^{2} A_{R e f}}
\end{aligned}
$$

\subsection{Wing Models}

Five delta wing models with 45 deg sweep angle having the same root chord length of $C=150 \mathrm{~mm}$ and $45 \mathrm{deg}$ bevel angle on the windward side of the wing, were manufactured using rapid prototyping of fine polyamide PA2200. The thicknesses of the wings were $3,5,7.5,15$, and $22.5 \mathrm{~mm}$ with the corresponding thickness-tochord ratios of $t / C=0.02,0.033,0.5,0.10$, and 0.15 , respectively. All of the wings, except the thinnest wing with $t / C=0.02$, had 24 pressure taps distributed symmetrically at the chordwise distance of $x / C=0.5$ and two smoke injection holes located at the apex of the wings. Due to the geometrical constraints, the $3 \mathrm{~mm}$ thick wing $(t / C=0.02)$ did not have any pressure taps nor smoke holes and was only used in Particle Image Velocimetry and force measurement experiments. Figure 3.7 demonstrates schematic of wing models geometries and location of the pressure and smoke holes over them.

\subsection{Experimental Matrices}

The effect of thickness-to-chord $(t / C)$ ratio on aerodynamics of a non-slender delta wing with sweep angle of 45 degree is characterized using laser illuminated smoke visualization, surface pressure measurements, particle image velocimetry, and force measurements. Laser illuminated smoke visualization experiments were performed for $t / C$ ratios of $0.033,0.05,0.10$, and 0.15 at Reynolds number of $R e=1.5 \times$ $10^{4}$ for the attack angles of $\alpha=6^{\circ}$ and $10^{\circ}$. Due to the dispersion of the injected 
smoke in high velocities, laser illuminated smoke visualization experiments were not conducted at higher Reynolds numbers. For pressure measurements, due to the high uncertainty of pressure values at relatively lower Reynolds numbers the experiments were conducted at Reynolds numbers of $R e=3.5 \times 10^{4}$ and $1 \times 10^{5}$ for the attack angles of $\alpha=6^{\circ}, 10^{\circ}, 14^{\circ}$ and $16^{\circ}$ for $t / C$ ratios of $0.033,0.05,0.10$, and 0.15 . In order to obtain preliminary insight for the wing with $t / C=0.02$ and for further characterization of $t / C$ ratio effect, crossflow PIV measurements at chordwise distance of $x / C=0.5$ were performed for the attack angles of $\alpha=10^{\circ}$ and $14^{\circ}$ at Reynolds number of $R e=3.5 \times 10^{4}$ and $1 \times 10^{5}$ for $t / C$ ratios of $t / C=0.02,0.033,0.05,0.10$, and 0.15 . Figure 3.8 represents the corresponding experimental matrices for flow visualization, pressure measurement, and PIV. Finally, force measurements were conducted for all five wings at Reynolds number of $R e=3.5 \times 10^{4}$ for angle of attacks ranging from 0 to 30 degrees. For the force measurements, since the strut and strain gauges were designed for the measurements of Reynolds number $R e=3.5 \times 10^{4}$, the system provides high uncertainties at Reynolds number higher than $R e=1 \times 10^{5}$.

\subsection{Uncertainty Estimates}

Uncertainty is an inevitable part of experimental research which may be created due to physical phenomenon or experimental apparatus, and uncertainty estimation is a vital part of any experimental research to confirm its accountability.

Equation 3.8 represents the calculation formula of resultant uncertainty, $\omega_{R}$, due to the uncertainty of each independent parameter, $\omega_{x_{i}}[70]$.

$$
\omega_{R}=\left[\left(\omega_{x_{1}} \frac{\partial R}{\partial x_{1}}\right)^{2}+\left(\omega_{x_{2}} \frac{\partial R}{\partial x_{2}}\right)^{2}+\cdots+\left(\omega_{x_{n}} \frac{\partial R}{\partial x_{n}}\right)^{2}\right]^{1 / 2}
$$

The following equation applied to calculate the relative uncertainty: 


$$
\frac{\omega_{R}}{R}=u_{R}
$$

Equation 3.8 was used to determine the uncertainty of surface pressure measurements related to pressure coefficients, where the pressure scanner have $0.003 \%$ FS accuracy. By applying Equation 3.8 to $C_{p}$ formula in Equation 3.1 one can prove that:

$$
\omega_{C_{p}}=\left[\left(\omega_{p} \frac{\partial C_{p}}{\partial \Delta P}\right)^{2}+\left(\omega_{p} \frac{\partial C_{p}}{\partial p_{d y n}}\right)^{2}\right]^{1 / 2}
$$

The differentiation results in:

$$
\omega_{C_{p}}=\left[\left(\frac{\omega_{p}}{p_{d y n}}\right)^{2}+\left(\frac{\omega_{p} \Delta P}{p_{d y n}{ }^{2}}\right)^{2}\right]^{1 / 2}
$$

The calculated relative uncertainty values for peak values of $-C_{p}$ are tabulated in Table 1. Appendix B represents the MATLAB code which have been used for the calculations. The relative uncertainty value for the pressure coefficient $-C_{p}$ were found to be less than $3.3 \%$ at the peak values.

The uncertainty of PIV measurements are determined within Insight 4G itself using the Peak Ratio (PR) uncertainty method. This method calculates the uncertainty values using peak to noise peak ratio (PPR), and in this method pixel displacement, seeding particles and pre-processing of the images are among the most important possible sources of error. Further details can be found in the Insight 4G Manual [71]. Table 2 represents the maximum uncertainty values for PIV measurements. The maximum uncertainty value is found to be $13.5 \%$. 
Table 1 Relative uncertainty values for the pressure measurements at the peak values for $t / C$ ratios of $0.033,0.05,0.10$, and 0.15 at $R e=35000$ and 100000 for angles of attack of $\alpha=6^{\circ}, 10^{\circ}, 14^{\circ}$ and $16^{\circ}$.

\begin{tabular}{|c|c|c|c|c|c|}
\hline \multicolumn{2}{|c|}{ Uncertainty (\%) } & $\mathrm{t} / \mathrm{C}=0.033$ & $\mathrm{t} / \mathrm{C}=0.05$ & $\mathrm{t} / \mathrm{C}=0.10$ & $\mathrm{t} / \mathrm{C}=0.15$ \\
\hline$\alpha=6^{\circ}$ & $\mathrm{Re}=35000$ & 2.9 & 3.12 & 2.73 & 2.71 \\
\hline$\alpha=10^{\circ}$ & $\mathrm{Re}=35000$ & 2.72 & 2.66 & 2.74 & 2.68 \\
\hline$\alpha=14^{\circ}$ & $\mathrm{Re}=35000$ & 2.81 & 2.85 & 2.98 & 3.03 \\
\hline$\alpha=16^{\circ}$ & $\mathrm{Re}=35000$ & 2.9 & 2.96 & 3.25 & 3.3 \\
\hline$\alpha=6^{\circ}$ & $\mathrm{Re}=100000$ & 0.33 & 0.33 & 0.31 & 0.31 \\
\hline$\alpha=10^{\circ}$ & $\mathrm{Re}=100000$ & 0.29 & 0.29 & 0.29 & 0.29 \\
\hline$\alpha=14^{\circ}$ & $\mathrm{Re}=100000$ & 0.33 & 0.33 & 0.37 & 0.39 \\
\hline$\alpha=16^{\circ}$ & $\mathrm{Re}=100000$ & 0.36 & 0.37 & 0.42 & 0.43 \\
\hline
\end{tabular}

Table 2 Maximum uncertainty values for the PIV measurements at $\mathrm{Re}=35000$ for angles of attack $\alpha=10^{\circ}$ and $14^{\circ}$.

\begin{tabular}{|c|c|c|c|c|c|c|}
\hline \multicolumn{2}{|c|}{ Uncertainty (\%) } & $\mathrm{t} / \mathrm{C}=0.02$ & $\mathrm{t} / \mathrm{C}=0.033$ & $\mathrm{t} / \mathrm{C}=0.05$ & $\mathrm{t} / \mathrm{C}=0.10$ & $\mathrm{t} / \mathrm{C}=0.15$ \\
\hline$\alpha=10^{\circ}$ & $\begin{array}{c}\mathrm{Re}=35000 \\
(3.5 \mathrm{~m} / \mathrm{s})\end{array}$ & 10.1 & 10.4 & 9.6 & 11 & 10 \\
\hline$\alpha=14^{\circ}$ & $\begin{array}{c}\mathrm{Re}=35000 \\
(3.5 \mathrm{~m} / \mathrm{s})\end{array}$ & 10.1 & 10.7 & 9.8 & 9.6 & 13.5 \\
\hline
\end{tabular}


For force measurements the uncertainty calculations were performed considering the uncertainties in the calibration, measured strain values, angle of attack, wind tunnel velocity, and air density. Appendix $\mathrm{C}$ represents the related formulas for uncertainty calculations. The maximum relative uncertainty values for $C_{L}$ and $C_{D}$ are found to be $6 \%$.

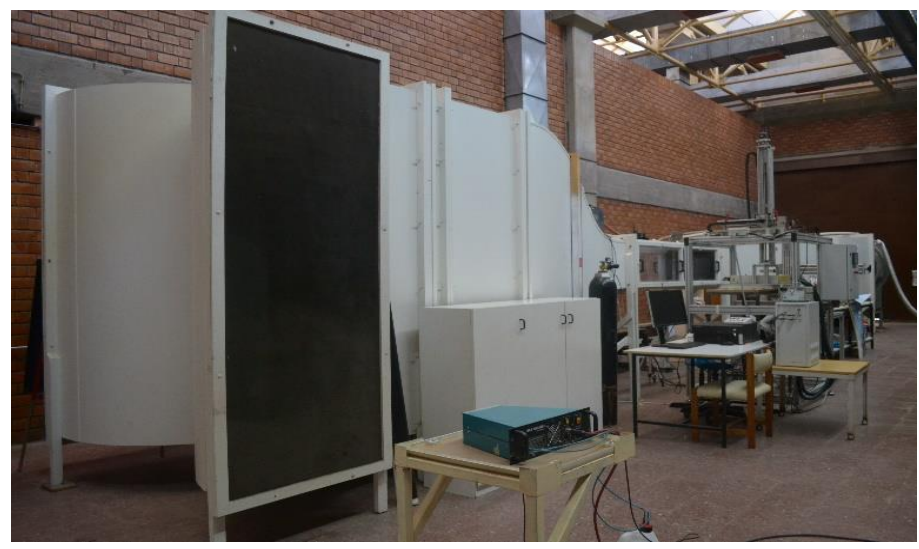

(a)

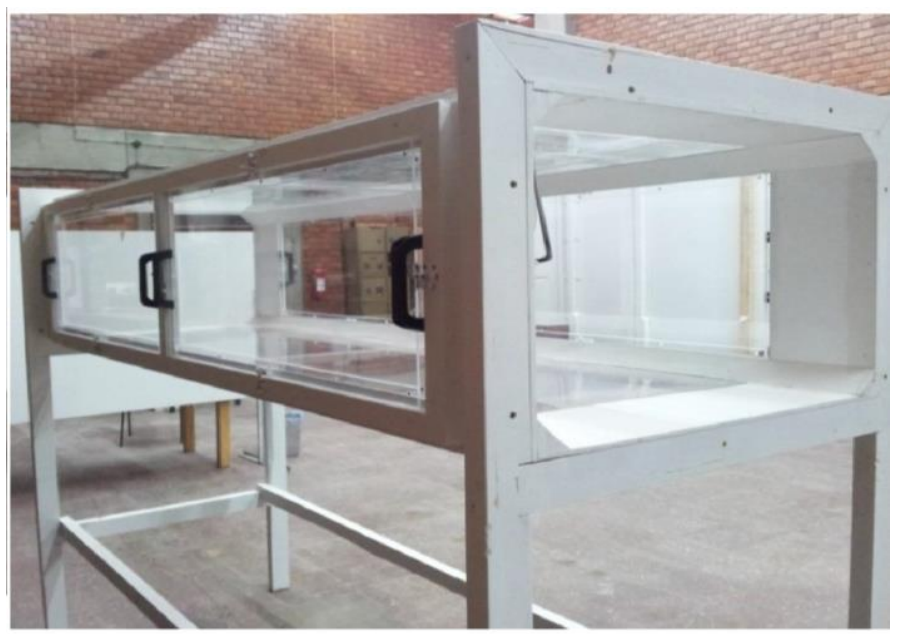

(b)

Figure 3.1 View from (a) wind tunnel facility and (b) test section. 


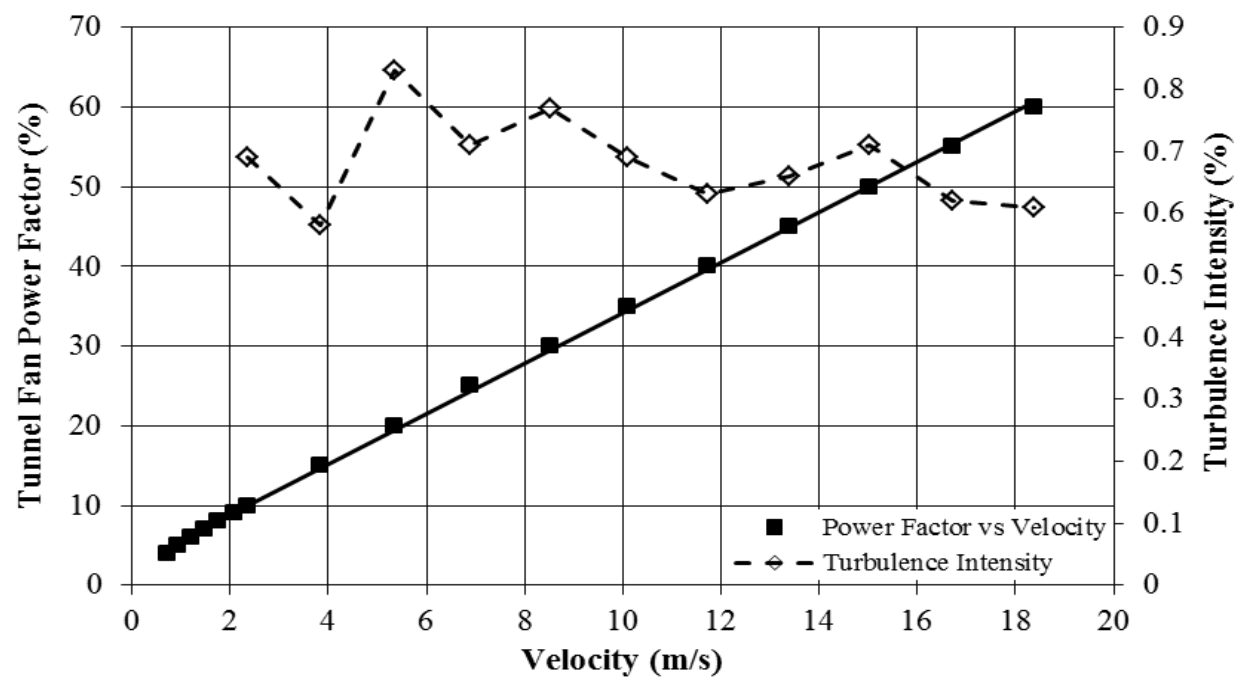

Figure 3.2 Tunnel fan power and turbulence intensity versus freestream velocity magnitudes.

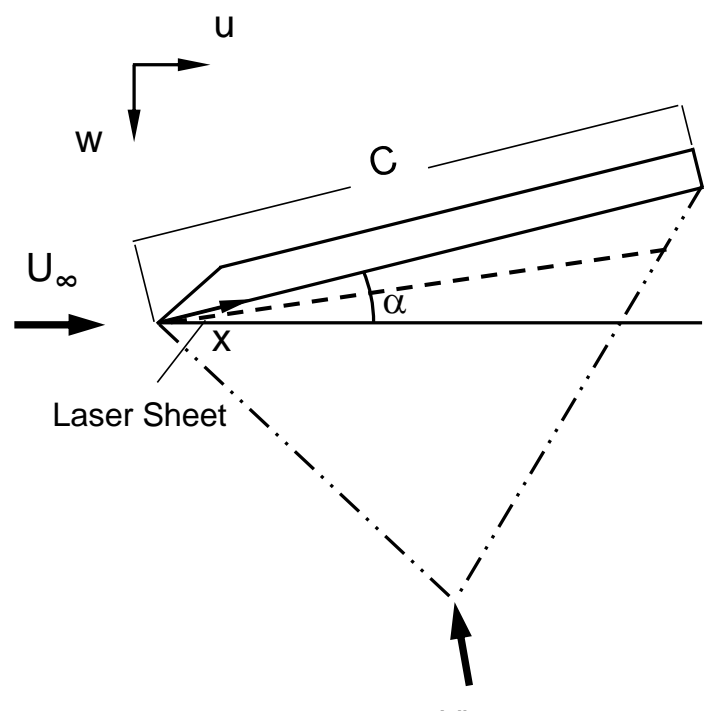

Surface Flow Visualization

Figure 3.3 Schematic representation of Surface flow visualization. 


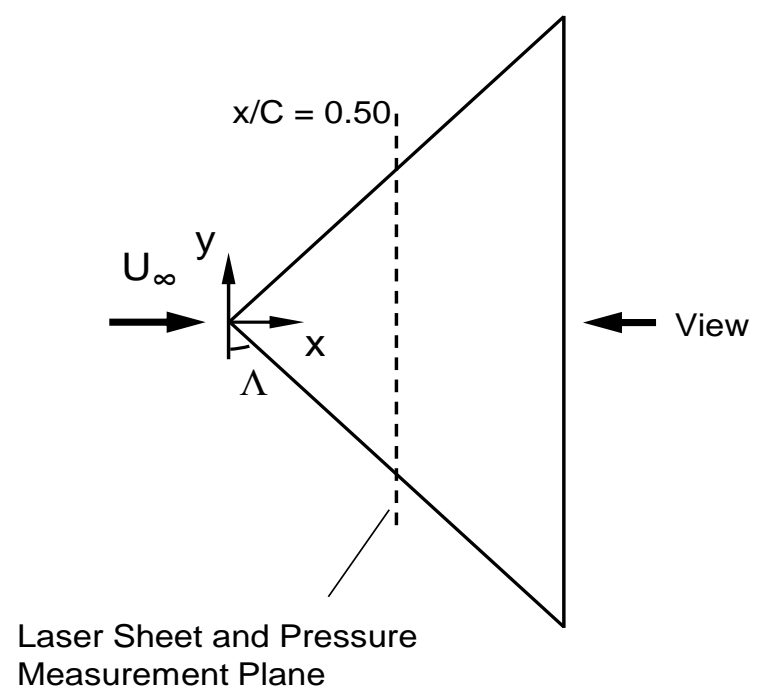

Pressure measurement \& PIV

Figure 3.4 Schematics representation of crossflow PIV laser sheet and pressure measurement plane.

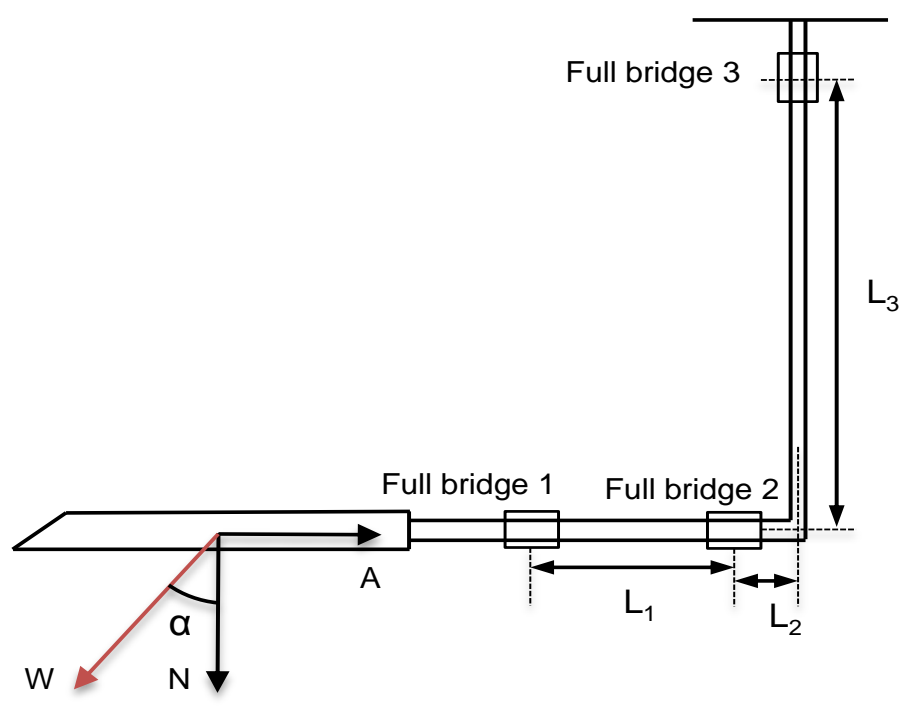

Figure 3.5 Schematic representation of force measurement system and corresponding normal to surface, $\mathrm{N}$, and axial, A, aerodynamic forces. 


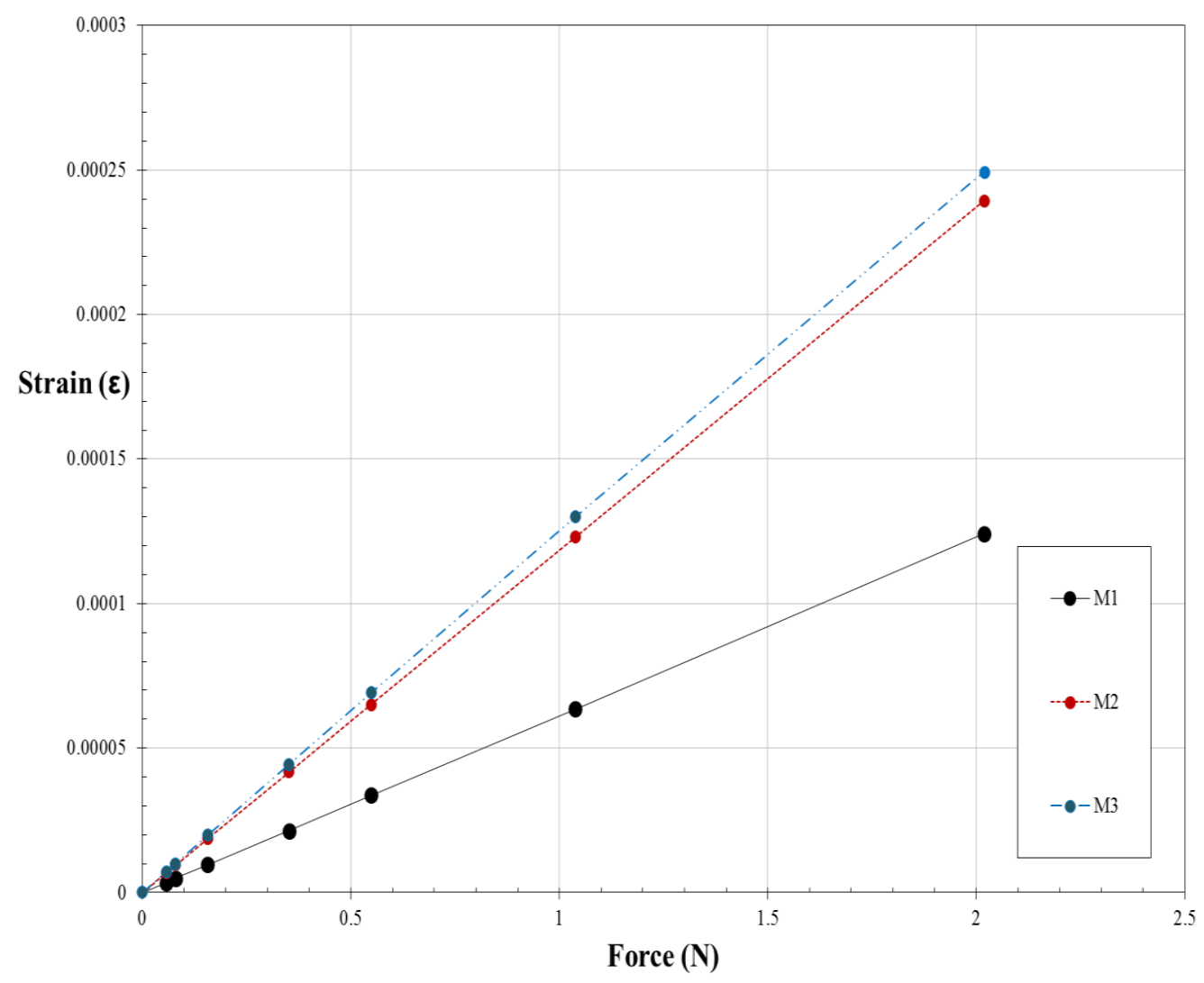

Figure 3.6 Calibration of full bridge strain gauges. 


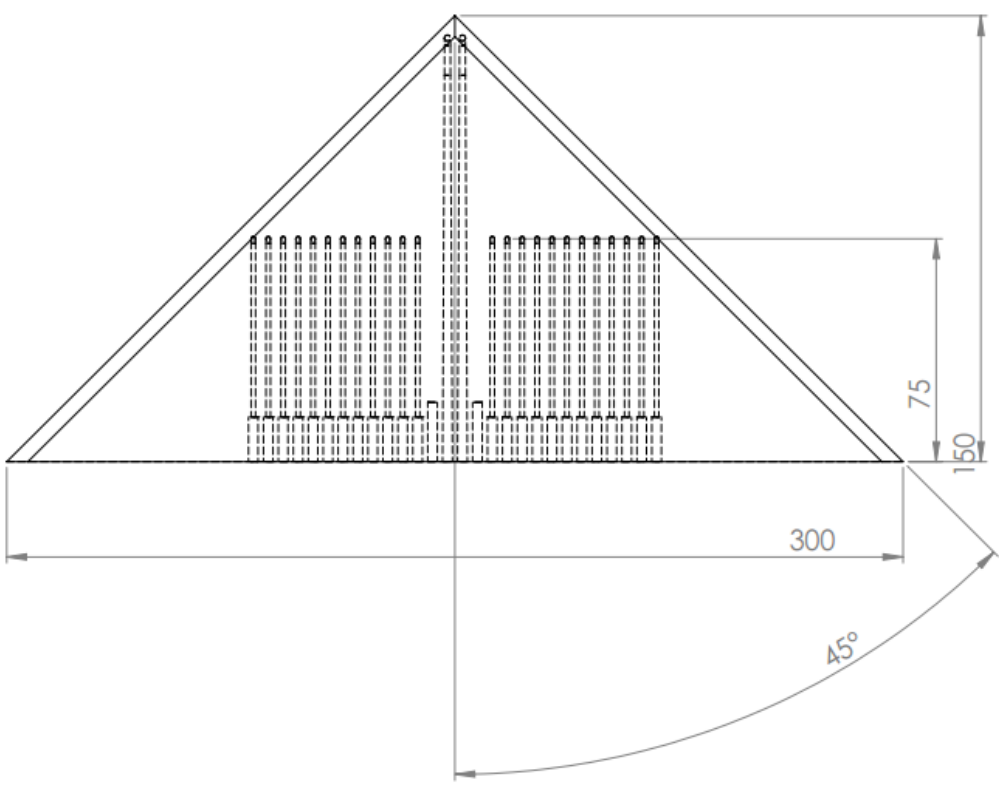

(a)

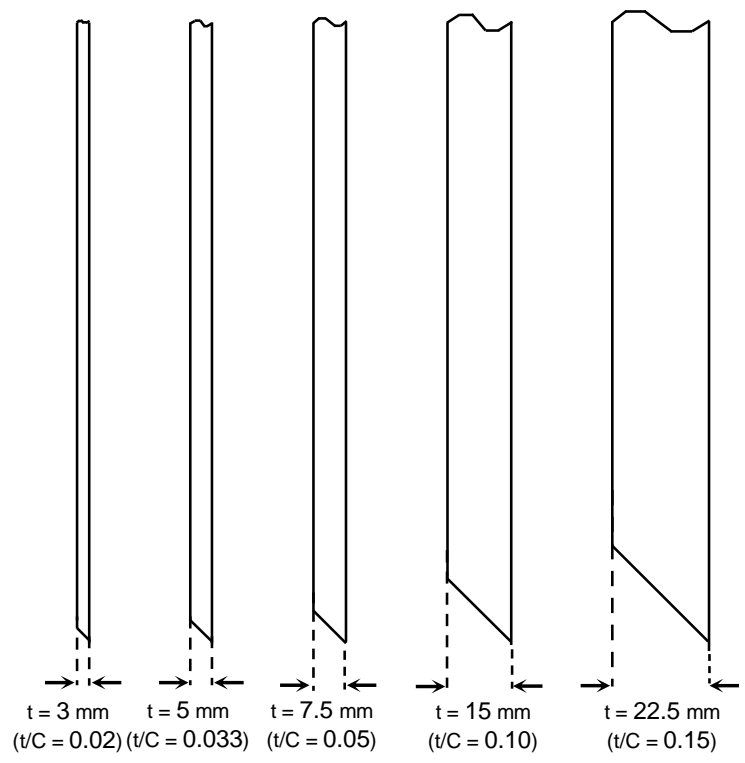

(b)

Figure 3.7 Schematic of: (a) geometrical dimensions and pressure and smoke holes of the wing models and (b) cross-sectional view of the wings and corresponding $t / C$ ratios. 


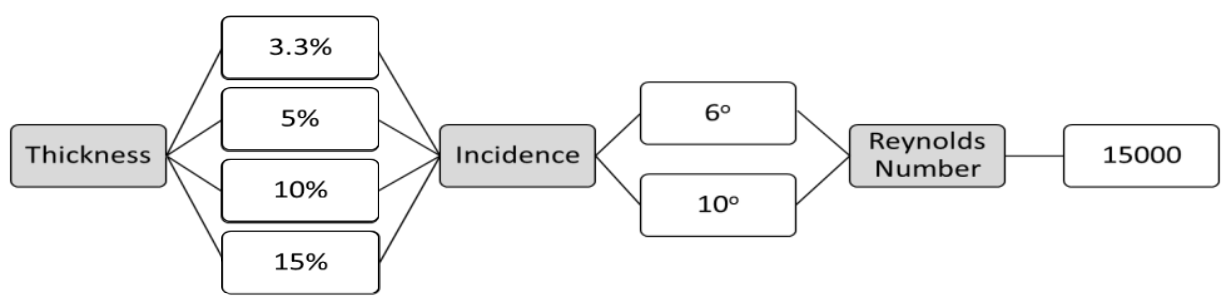

(a)

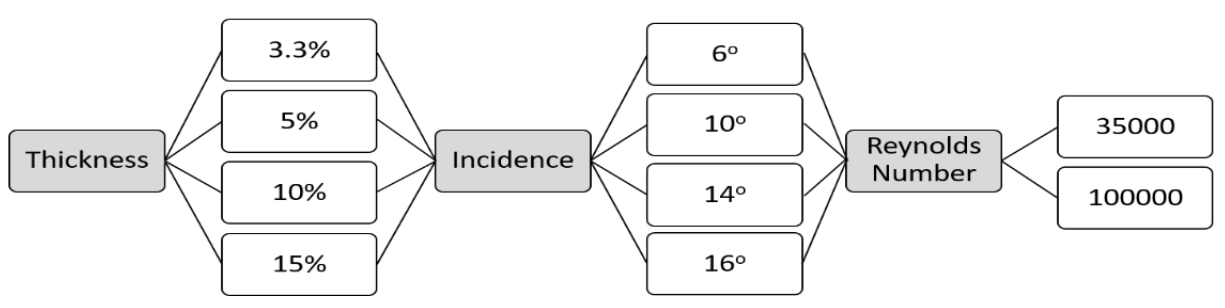

(b)

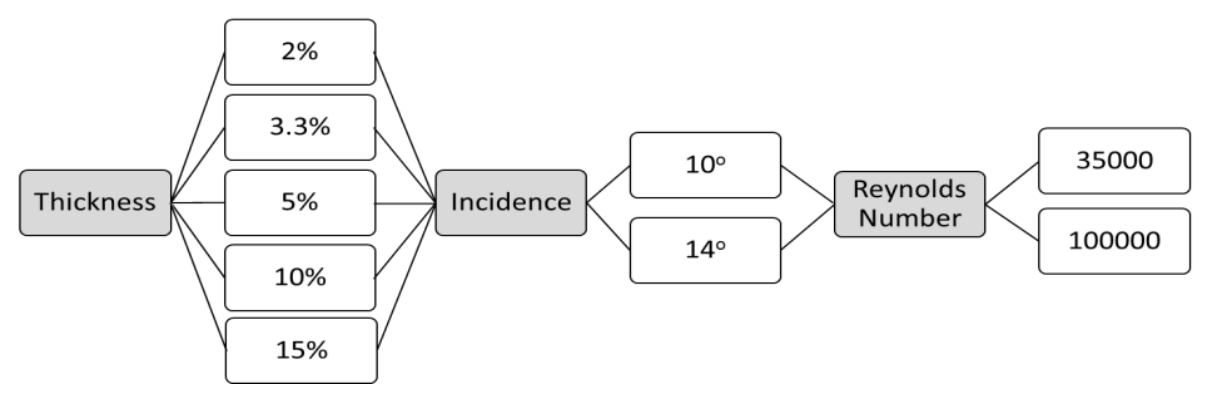

(c)

Figure 3.8 Experimental matrices for (a) surface laser illuminated flow smoke visualizations (b) pressure measurements and (b) PIV measurements. 


\section{CHAPTER 4}

\section{RESULTS AND DISCUSSION}

\subsection{The Results of Surface Flow Visualizations}

Figures 4.1 and 4.2 demonstrate the surface flow smoke visualization results for $t / C$ ratios of $0.033,0.05,0.10$, and 0.15 at Reynolds number of $R e=1.5 \times 10^{4}$ for the attack angles of $\alpha=6^{\circ}$ and $10^{\circ}$, respectively. In both figures, surface smoke visualization of each $t / C$ ratio wing is presented from top to bottom in ascending order, except the wing with $t / C=0.02$, which do not have any smoke injection holes and thus the corresponding smoke visualizations due to the geometrical constraints as explained in the experimental set-up section.

Considering the top image in Figure 4.1, which displays the surface flow visualization of $t / C=0.033$ at angle of attack $\alpha=6^{\circ}$, the smoke traces indicate a dual vortex structure, where the vortex breakdown of the first primary vortex takes place in the vicinity of the trailing edge and the second primary vortex breaks down upstream closer to the mid-chord. When the $t / C$ ratio reaches 0.05 , second primary vortex disappears and common form of leading edge vortex without indication of breakdown is evident. As the $t / C$ ratio increases to 0.10 vortex breakdown appears in proximity to the mid-chord, and further increase of $t / C$ ratio to 0.15 , moves the breakdown location upstream toward the apex. The overall evaluation of Figure 4.1 indicates that the formation of leading edge vortex structure and its corresponding breakdown location are considerably affected by the thickness-to-chord ratio.

Figure 4.2 represents the results of surface flow smoke visualizations for angle of attack $\alpha=10^{\circ}$. Considering the smoke traces of $t / C$ ratios of 0.033 and 0.05 as shown at the top two row of Figure 4.2, a leading edge vortex with a breakdown in proximity to mid-chord is evident. Both cases indicate quite similar flow structure 
and breakdown locations at angle of attack $\alpha=10^{\circ}$ in contrast to the aforementioned smoke visualizations for angle of attack $\alpha=6^{\circ}$ where $t / C=0.033$ and 0.05 wings demonstrate different leading-edge vortex formations as indicated in Figure 4.1. Further increase in $t / C$ ratio to 0.10 and then to 0.15 at angle of attack $\alpha=10^{\circ}$ as shown at the bottom two rows of Figure 4.2, causes movement of vortex breakdown location upstream toward the apex of the wing. 

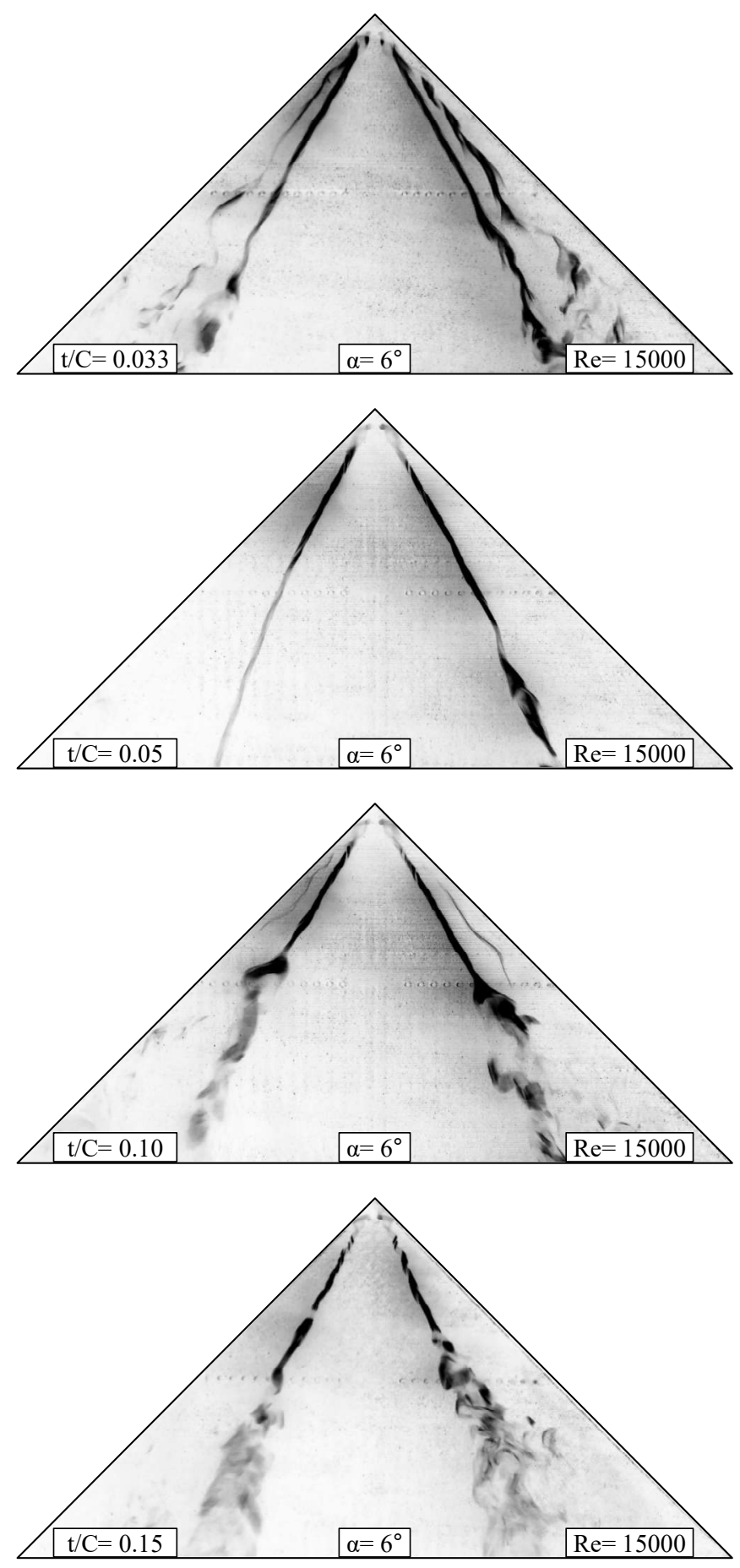

Figure 4.1 Laser-illuminated surface flow smoke visualizations of $t / C=$ $0.033,0.05,0.10$ and 0.15 for $R e=1.5 \times 10^{4}$ at angle of attack of $\alpha=6 \mathrm{deg}$. 

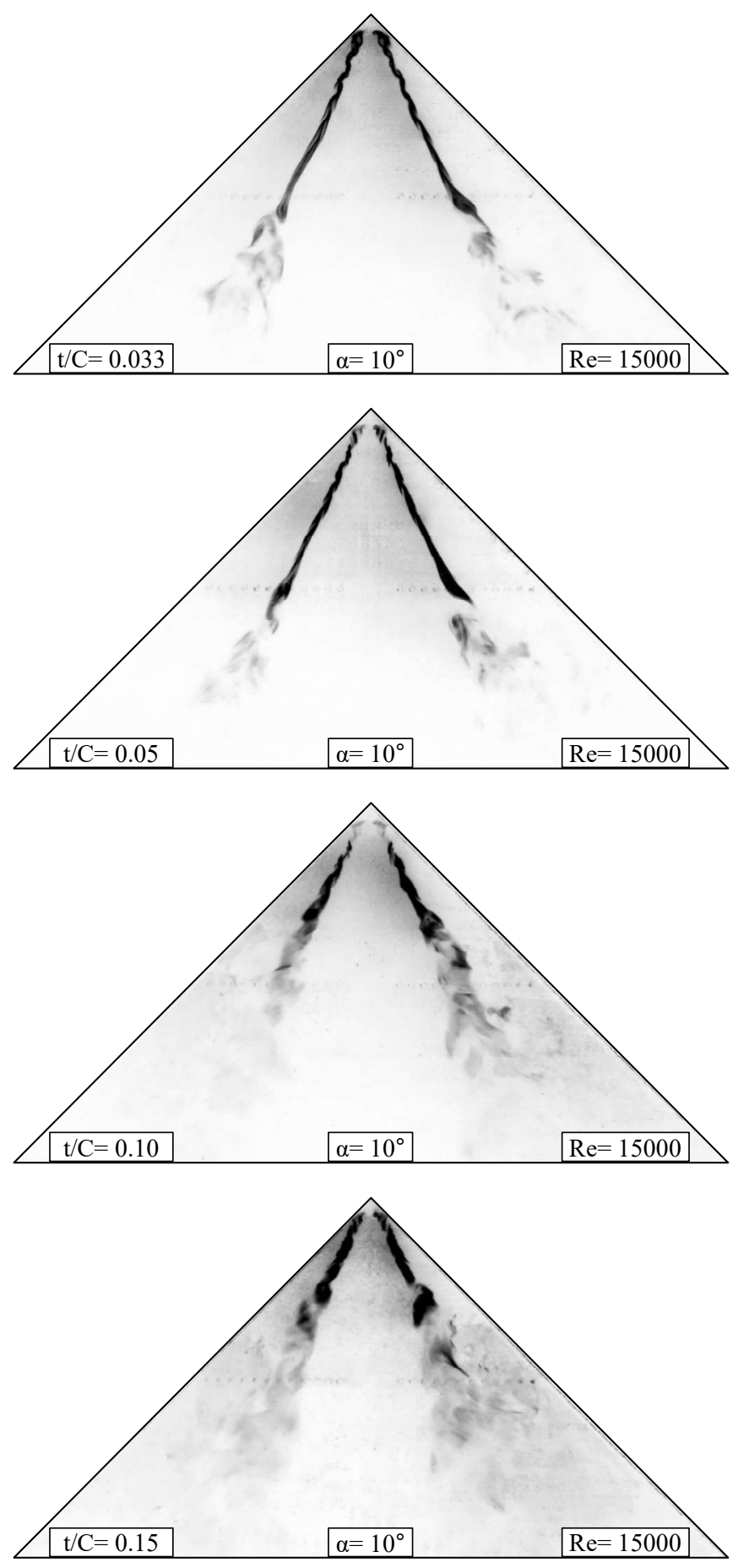

Figure 4.2 Laser-illuminated surface flow smoke visualizations of $t / C=$ $0.033,0.05,0.10$ and 0.15 for $R e=1.5 \times 10^{4}$ at angle of attack of $\alpha=10 \mathrm{deg}$. 


\subsection{The Results of Surface Pressure Measurements}

The results of mean surface pressure measurements in terms of dimensionless pressure coefficient $-C_{p}$ at chordwise distance of $x / C=0.5$ for Reynolds numbers $R e=3.5 \times 10^{4}$ and $1 \times 10^{5}$ are demonstrated in Figures 4.3 and 4.4, respectively. The $-C_{p}$ distributions are presented for $t / C$ ratios of $0.033,0.05,0.10$, and 0.15 , except for the wing with $t / C=0.02$, which do not have any pressure taps and thus the corresponding pressure distributions due to the geometrical constraints as explained in the experimental set-up section. Each chart corresponds to different angle of attack, $\alpha=6^{\circ}, 10^{\circ}, 14^{\circ}$ and $16^{\circ}$ listed from top left to bottom right in ascending order. The horizontal axis of the chart represents the dimensionless spanwise distance from the centerline, $y / S$, for which the $S$ is the half-span at $x / C=0.5$. High $-C_{p}$ values in the charts indicate suction and possible projected region of vortex core on the wing surface, whereas, low $-C_{p}$ values indicate the region where the flow possibly attaches to the wing surface.

Figure 4.3 demonstrates the $-C_{p}$ distributions for Reynolds number $R e=3.5 \times$ $10^{4}$. Considering the results for the angle of attack $\alpha=6^{\circ}$ as shown in the top left chart, the pressure distribution for each $t / C$ ratio wing exhibits hump-like pattern, which is considered as the footprint of the leading edge vortex structure. As the $t / C$ ratio increases, increase in maximum $-C_{p}$ value, which is an indication of stronger suction, and slight movement of the location of the peak value toward the centerline are evident. Considering the $-C_{p}$ distributions for $\alpha=10^{\circ}$ as indicated at the bottom left of Figure 4.3, the results demonstrate the pattern of vortical structure for all $t / C$ ratio wings with similar peak $-C_{p}$ behavior in terms of magnitude and location. Slightly lower minimum $-C_{p}$ values for low $t / C$ ratio wings, $t / C=0.033$ and 0.05 , are apparent, which can be interpreted as stronger reattachment to the surface for these wing models. In addition, the $-C_{p}$ distributions for $t / C=0.033$ and 0.05 wings are almost identical, which is quite in line with the aforementioned surface smoke visualizations for these wings at angle of attack $\alpha=10^{\circ}$ and 
Reynolds number $R e=1.5 \times 10^{4}$ as indicated in Figure 4.2, where the leading edge vortex structures and the breakdown locations appear to be quite similar. Considering $-C_{p}$ results for attack angle of $\alpha=14^{\circ}$, shown in the top right chart of Figure 4.3, the pressure distribution demonstrates the footprint of vortical structure on the wing surface for $t / C=0.033$ and 0.05 . As the $t / C$ ratio increases, the pressure distribution turns into more flat profile where the difference in the magnitude of the highest and the lowest $-C_{p}$ value, $\Delta C_{p}$, drops significantly. This is an indication of drop in the strength of leading edge vortex. For the angle of attack $\alpha=16^{\circ}$, which is shown at bottom right of Figure 4.3, complete flat-like $-C_{p}$ distribution is apparent for $t / C$ of 0.10 and 0.15 , which is an indication of threedimensional separated flow over the wing surface. All these observations for surface pressure distributions are quite in line with the results of recent study of Gulsacan et al. [8] in which the effect of thickness-to-chord ratio on flow structure of a low swept delta wing is reported.

To further discuss the global effect of thickness-to-chord ratio on flow structure and its sensitivity to Reynolds number, the $-C_{p}$ distributions for Reynolds number $R e=1 \times 10^{5}$ are demonstrated in Figure 4.4. Without discussing the details of each chart in Figure 4.4, the global comparison of two figures, Figures 4.3 and 4.4, demonstrate that the charts of both figures have very similar pressure distributions, which indicate that the Reynolds number dependency for the effect of thickness to chord ratio on flow structure is quite minimal. The only remarkable difference observed when comparing the results of different Reynolds numbers as demonstrated in Figures 4.3 and 4.4 is that $t / C=0.033$ and 0.05 wings induce almost identical surface pressure distributions for all angles of attack at $R e=1 \times$ $10^{5}$, whereas this is apparent for only angle of attack $\alpha=10^{\circ}$ at $R e=3.5 \times 10^{4}$. The general assessment of pressure measurement results in Figures 4.3 and 4.4 for both Reynolds numbers of $3.5 \times 10^{4}$ and $1 \times 10^{5}$ reveals that for low angles of attack, the strength of the leading edge vortex increases as the $t / C$ ratio increases, 
whereas for high angles of attack, three dimensional surface separation indicated by flat pressure distribution appears at relatively lower angle of attack as the $t / C$ ratio increases. Furthermore, for the angle of attack $\alpha=10^{\circ}$, all $t / C$ ratio wings induce quite similar pressure distributions on the wing surface. 

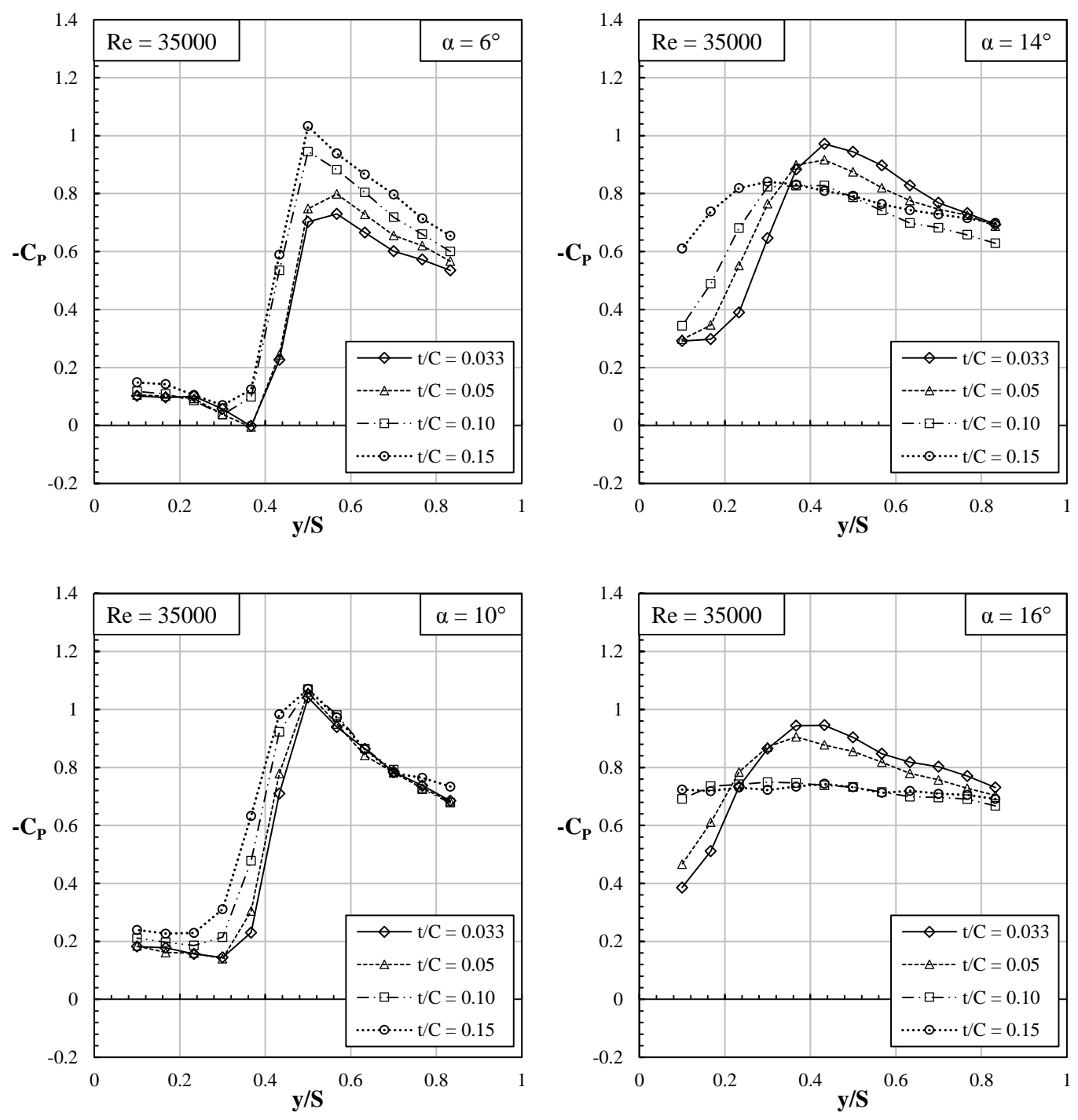

Figure 4.3 The dimensionless pressure distribution $-C_{p}$ with respect to dimensionless half span for $t / C=0.033,0.05,0.10$, and 0.15 for $R e=3.5 \times$ $10^{4}$ at angles of attack of $\alpha=6,10,14$, and 16 degrees. 

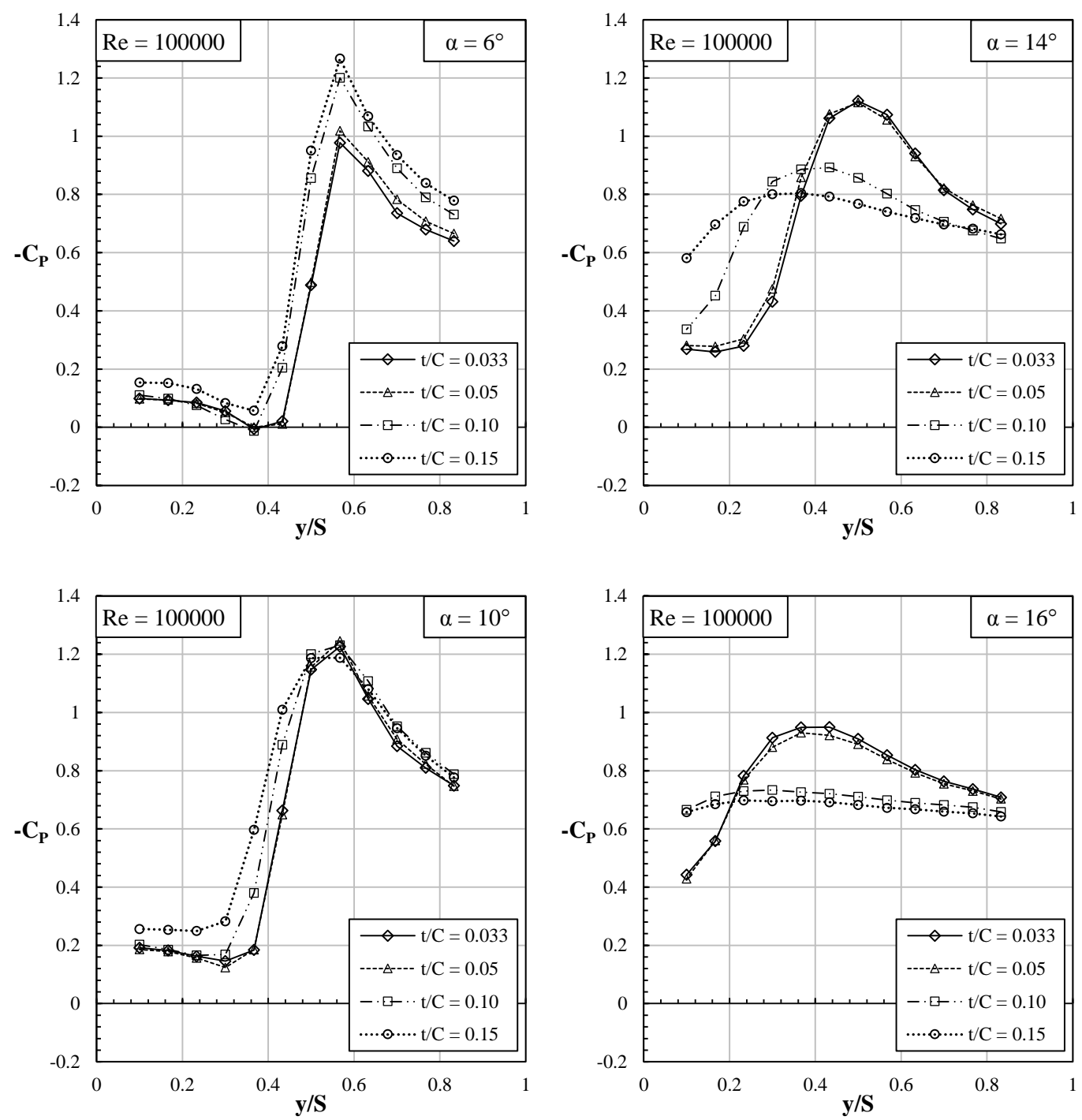

Figure 4.4 The dimensionless pressure distribution $-C_{p}$ with respect to dimensionless half span for $t / C=0.033,0.05,0.10$, and 0.15 for $R e=1 \times$ $10^{5}$ at angles of attack of $\alpha=6,10,14$, and 16 degrees. 


\subsection{The Results of Crossflow PIV Measurements}

In order to further characterize the effect of $t / C$ ratio on flow structure and to provide preliminary insight for the wing $t / C=0.02$, the crossflow PIV results at chordwise distance of $x / C=0.5$ for the attack angles of $\alpha=10^{\circ}$ and $14^{\circ}$ at Reynolds number of $R e=3.5 \times 10^{4}$ and $1 \times 10^{5}$ for $t / C$ ratios of $t / C=$ $0.02,0.033,0.05,0.10$, and 0.15 are demonstrated in Figures 4.5 and 4.6, respectively. In selection of these cases, the results of surface pressure measurements were taken into consideration. For both Figures 4.5 and 4.6, the results for attack angles of $\alpha=10^{\circ}$ and $14^{\circ}$ are shown in the left and right columns, respectively. In each column, the time-averaged velocity vectors $\langle V\rangle$, and the constant contours of time averaged axial nondimensional vorticity $\langle\omega C / U\rangle$ are illustrated at the left and right side of the wing, respectively, and the pattern of each $t / C$ ratio wing is presented from top to bottom in ascending order. For the constant contours of time averaged nondimensional axial vorticity $\langle\omega C / U\rangle$, the solid (dark) and dashed (light) lines display positive and negative contours, respectively, in which the positive direction defined as inward normal direction. The absolute minimum and the absolute incremental values of constant contours of time averaged nondimensional axial vorticity are both set as 4 for all cases to allow one-to-one comparison. $\left([|(<\omega C / U>)|]_{\min }=4\right.$ and $\left.[\Delta|(<\omega C / U>)|]=4\right)$.

Considering the results for the angle of attack $\alpha=10^{\circ}$ at $R e=3.5 \times 10^{4}$ as demonstrated in the left column of Figure 4.5, both the time averaged velocity vectors and the vorticity contours indicate that all $t / C$ ratio wings induce quite similar vortical flow pattern in terms of both the qualitative and the quantitative evaluations. As the $t / C$ ratio increases, there is a slight increase in the spatial extent of the overall vorticity concentration along with the movement of the structure toward the centerline, which in turn causes a slight reduction in the localized strength of the vorticity. 
The velocity vectors and the vorticity contours for the angle of attack $\alpha=14^{\circ}$ at $R e=3.5 \times 10^{4}$ are indicated in the right column of Figure 4.5. The results indicate that effect of $t / C$ ratio on flow structure over the wing is substantial. As the $t / C$ ratio increases, significant reduction in the levels of vorticity and the movement of the vortex structure inboard toward the symmetry plane are evident. At the highest $t / C$ ratio, the vortical structure deteriorates and the reattachment region appears in proximity to the mid plane. All these cross-flow observations for the angles of attack $\alpha=10^{\circ}$ and $\alpha=14^{\circ}$ are quite in line with the corresponding surface pressure distributions demonstrated in Figure 4.3.

In order to verify the independency of the effect of thickness-to-chord ratio on flow topology from Reynolds number, which was represented in pressure measurement results, the time averaged velocity vectors and the vorticity contours for Reynolds number of $R e=1 \times 10^{5}$ at the angle of attack of $\alpha=10^{\circ}$ and $14^{\circ}$ are demonstrated in Figure 4.6. Without discussing the details of each chart, general comparison of Figures 4.5 and 4.6 indicates that the effect of $t / C$ ratio on velocity vectors and vorticity contours is almost identical, and the effect of $t / C$ ratio on flow structure is similar in both Reynolds numbers of $R e=3.5 \times 10^{4}$ and $1 \times 10^{5}$.

In order to characterize the effect of $t / C$ ratio on unsteady behavior of the flow and the velocity fluctuations, the contours of constant nondimensional rms of normal to surface component of velocity $w_{r m s} / U$ and transverse component of velocity $v_{r m s} / U$ for the attack angles of $\alpha=10^{\circ}$ and $14^{\circ}$ at Reynolds number of $R e=$ $3.5 \times 10^{4}$ for $t / C$ ratios of $t / C=0.02,0.033,0.05,0.10$, and 0.15 are demonstrated in Figures 4.7. The results for attack angles of $\alpha=10^{\circ}$ and $14^{\circ}$ are shown in the left and right columns of Figure 4.7, respectively. Each column contains the contours of constant non-dimensional rms of transverse component of velocity $v_{r m s} / U$ and normal to surface component of velocity $w_{r m s} / U$ at the left and right side of the wing, respectively. The absolute minimum and the absolute incremental values of constant contours are set as 0.1 and 0.02 for all cases to allow one-to-one comparison. The results for $\alpha=10^{\circ}$ in left column of Figure 4.7 show 
that as $t / C$ ratio increases spatial extend of both the $v_{r m s} / U$ and $w_{r m s} / U$ contours increases, However the movement of contours toward the symmetry plane is quite minimal and the contours levels are almost unaffected by $t / C$ ratio. For $\alpha=14^{\circ}$ in right column of Figure 4.7, as the $t / C$ ratio increases, spatial extension of both the $v_{r m s} / U$ and $w_{r m s} / U$ contours are evident. The levels of contours of $v_{r m s} / U$ are increased with the increase of $t / C$ ratio, but for $w_{r m s} / U$, the levels of contours remain almost unaffected. 

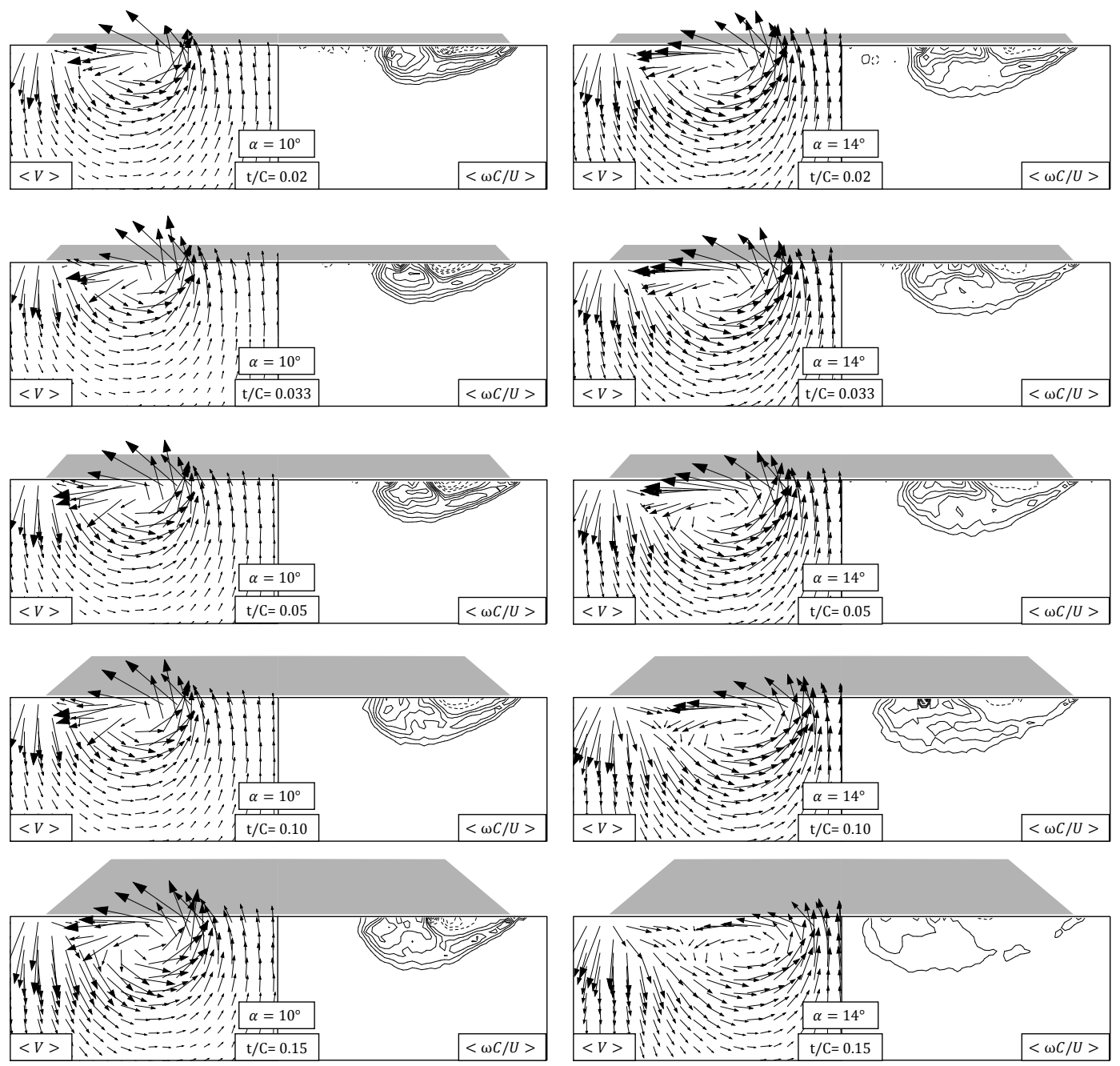

Figure 4.5 The time-averaged velocity vectors $\langle V\rangle$ and constant contours of nondimensional axial vorticity $\langle\omega C / U\rangle$ at angles of attack $\alpha=10$ (left column) and 14 (right column) degrees and $R e=3.5 \times 10^{4}$ for $t / C=0.02,0.033,0.05,0.10$, and 0.15: $[|\langle\omega C / U\rangle|]_{\min }=4, \Delta[|\langle\omega C / U\rangle|]=4$. 

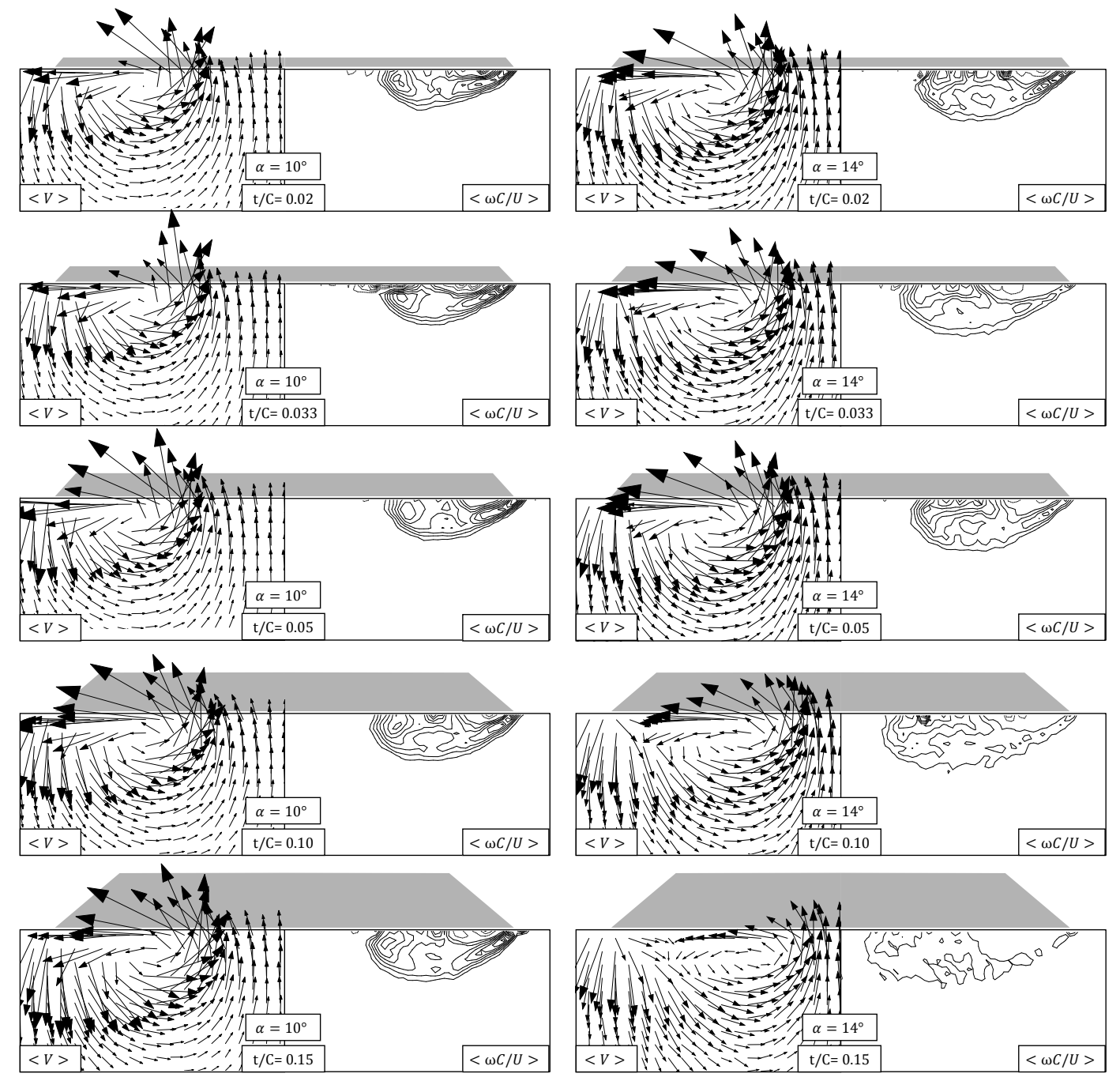

Figure 4.6 The time-averaged velocity vectors $\langle V\rangle$ and constant contours of nondimensional axial vorticity $\langle\omega C / U\rangle$ at angles of attack $\alpha=10$ (left column) and 14 (right column) degrees and $R e=1 \times 10^{5}$ for $t / C=0.02,0.033,0.05,0.10$, and 0.15: $[|\langle\omega C / U\rangle|]_{\min }=4, \Delta[|\langle\omega C / U\rangle|]=4$. 

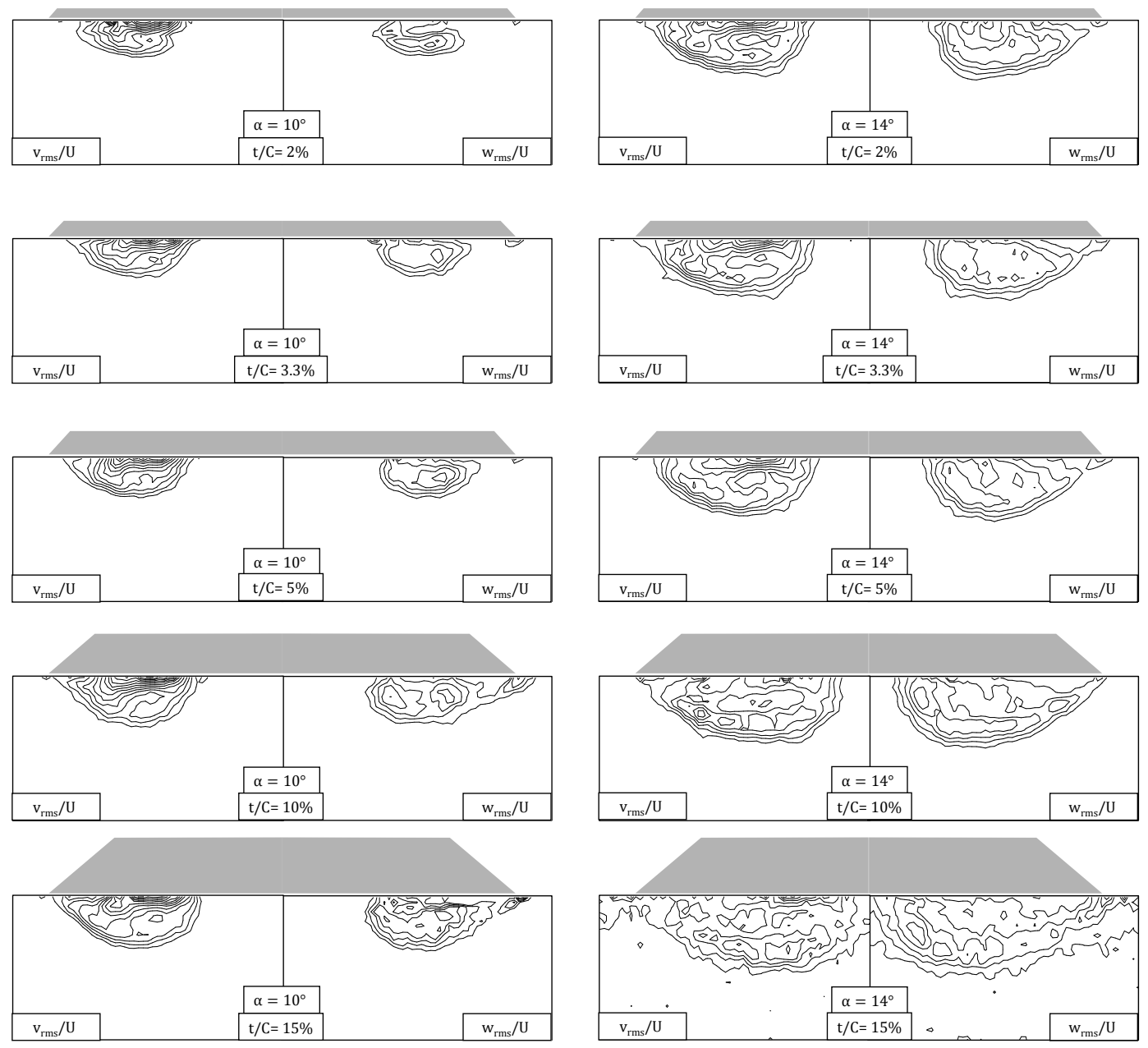

Figure 4.7 Contours of constant non-dimensional rms of normal to surface component of velocity $w_{r m s} / U$ and transverse component of velocity $v_{r m s} / U$ at angles of attack $\alpha=10$ (left column) and 14 (right column) degrees and $R e=$ $3.5 \times 10^{4}$ for $t / C=0.02,0.033,0.05,0.10$, and $0.15:\left[\left|\left\langle v_{r m s} / U\right\rangle\right|\right]_{\min }=0.1$, $\left[\left|\left\langle w_{r m s} / U\right\rangle\right|\right]_{\min }=0.1, \Delta\left[\left|\left\langle v_{r m s} / U\right\rangle\right|\right]=0.02, \Delta\left[\left|\left\langle w_{r m s} / U\right\rangle\right|\right]=0.02$. 


\subsection{The Results of Force Measurements}

In order to characterize the effect of $t / C$ ratio on aerodynamic performance of the wing and to complement the velocity and the pressure measurements as mentioned in previous sections, the drag and lift force measurements were conducted for $t / C$ ratios of $0.02,0.033,0.05,0.10$, and 0.15 . The results of force measurements for a broad range of angles of attack varying from 0 to 30 degrees at Reynolds number of $R e=3.5 \times 10^{4}$ are demonstrated in Figure 4.8, where the lift coefficient $C_{L}$, drag coefficient $C_{D}$, and the corresponding Lift-to-Drag ratio $C_{L} / C_{D}$ charts are listed from top to bottom, respectively.

Considering the $C_{L}$ distributions demonstrated in the top chart of Figure 4.8, $C_{L}$ for $t / C=0.02$ rises with a sharp slope up to the value of 0.86 at angle of attack $\alpha=18^{\circ}$, where the wing experiences significant drop in $C_{L}$ with a clear indication of stall afterwards. For the cases of $t / C$ ratio $0.033,0.05$, and 0.10 , the maximum lift coefficients, $C_{L \max }$, reach to $0.74,0.71$, and 0.63 , respectively, where the slopes of corresponding $C_{L}$ curves change at angle of attack $\alpha=12^{\circ}$ and drops in lift coefficients start at the angle of attack of $16^{\circ}$ in these cases. For the wing with $t / C=0.15$, the sharp slope of $C_{L}$ curve decays significantly once the angle of attack exceeds $\alpha=8^{\circ}$, and $C_{L}$ gradually increases up to the angle of attack $\alpha=16^{\circ}$ to the value $C_{\operatorname{Lmax}}=0.56$. In addition, all the $t / C$ ratio wings induce steady $C_{L}$ distributions around the corresponding $C_{L \max }$ values for a broad range of angle of attack except the wing with $t / C=0.02$, where a distinctive $C_{\text {Lmax }}$ following with a significant drop in $C_{L}$ value is evident.

The overall evaluation of $C_{L}$ curves demonstrate that the $C_{L m a x}$ values decrease substantially as the $t / C$ ratio increases. Furthermore, drop in the slope of $C_{L}$ curve appears at relatively lower angle of attack for high $t / C$ ratio wings. However, at low angles of attack, high $t / C$ ratio wings induce higher lift coefficients. These observations are quite in line with the results of surface pressure measurements as indicated in Figure 4.3, such that the strength of the vortex structure increases as 
the $t / C$ ratio increases at low angles of attack, whereas high $t / C$ ratio wings have flat pressure distributions indicating three-dimensional surface separations at relatively lower angle of attack compared to low $t / C$ ratio wings.

The corresponding $C_{D}$ values for $t / C$ ratios of $0.02,0.033,0.05,0.10$, and 0.15 , at Reynolds number of $R e=3.5 \times 10^{4}$ are demonstrated in the middle chart of Figure 4.8. The drag coefficient $C_{D}$ of all $t / C$ ratio wings increase with increase in angle of attack as expected. The general patterns of the $C_{D}$ curves indicate substantial rise in drag coefficients as the $t / C$ ratio increases with a few exceptional cases including $t / C$ ratios of 0.033 and 0.05 .

To further characterize the effect of $t / C$ ratio on aerodynamic performance of the wing, the $C_{L} / C_{D}$ values are plotted and demonstrated in the bottom chart of Figure 4.8. The results indicate that reduction in $t / C$ ratio causes shift in lift-to-drag ratio $C_{L} / C_{D}$ distributions to higher levels in general, with substantial increase in maximum $C_{L} / C_{D}$ value. Each $t / C$ ratio wing has its corresponding maximum $C_{L} / C_{D}$ value at different angle of attack. In addition, as the $t / C$ ratio increases, $C_{L} / C_{D}$ distributions turn into more flat profile for a wide range of angles of attack. It is also important to emphasize that all $t / C$ ratio wings induce identical $C_{L} / C_{D}$ values at the angle of attack of $\alpha=0^{\circ}$.

Considering the results of Kawazoe et al. [9] for 45 deg swept delta wing with circular leading edge, the thicker wing represents more resistance to three dimensional separation in the pressure measurement results and higher stall angle in the $C_{L}$ curve, which deviates from the results of the present study. This might imply that the shape of leading edge is critical in determination of $t / C$ ratio effect over the flow structure and aerodynamic performance of a non-slender delta wing. 

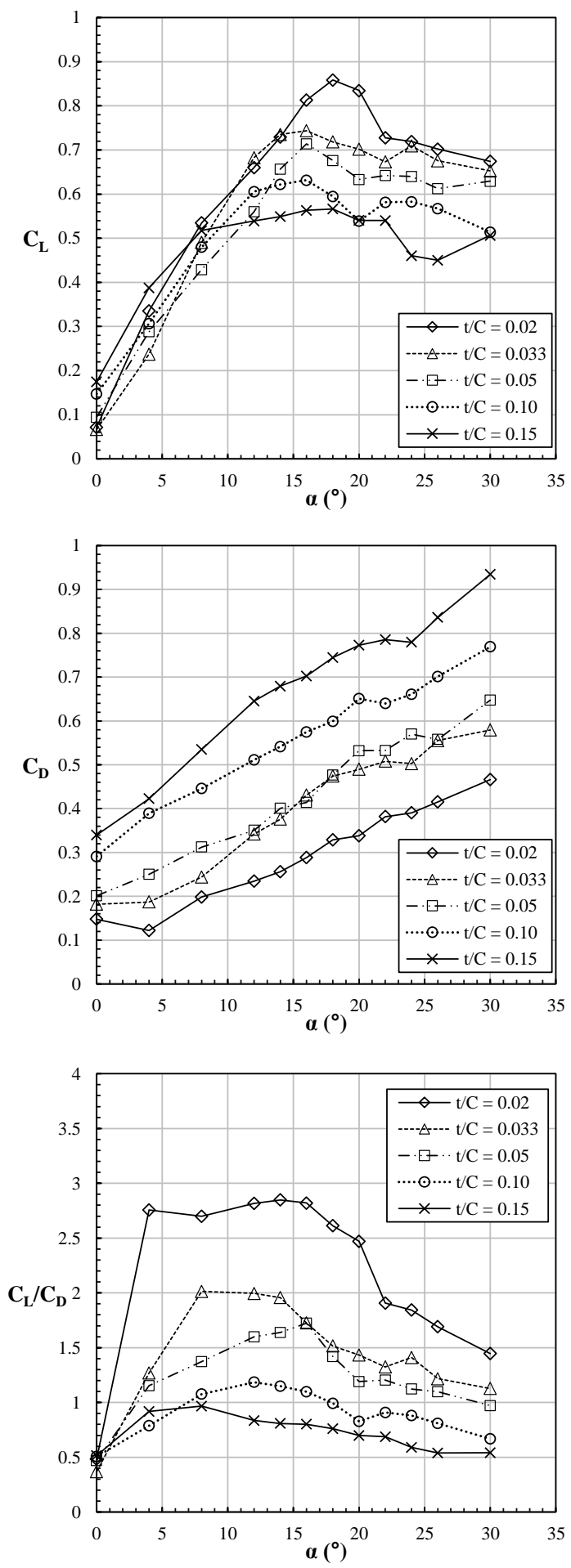

Figure 4.8 Lift coefficient $C_{L}$ (top), Drag coefficient $C_{D}$ (middle), and Lift-to-Drag ratio $C_{L} / C_{D}$ (bottom) with respect to angle of attack for $t / C=$ $0.02,0.033,0.05,0.10$, and 0.15 at $R e=3.5 \times 10^{4}$. 


\section{CHAPTER 5}

\section{CONCLUSION}

\subsection{Summary and Conclusions}

In the present study, the effect of thickness-to-chord ratio on overall flow structure and aerodynamic performance of a 45 deg swept delta wing is investigated using laser-illuminated smoke visualization, surface pressure measurement, particle image velocimetry (PIV), and force measurements. Experiments are carried out in a low speed wind tunnel using delta wing planforms with $t / C$ ratios $2,3.3,5,10$, and $15 \%$ for Reynolds numbers varying from $R e=1.5 \times 10^{4}$ to $1 \times 10^{5}$ and angles of attack varying from 0 to 30 degrees. Considering the combined assessments of the velocity, pressure, and force measurements, the main conclusions can be summarized as follow:

- The effect of $t / C$ ratio on flow structure is substantial such that the formation of leading edge vortex and its corresponding breakdown location are considerably influenced by the $t / C$ ratio. At relatively lower angle of attacks, the strength of the vortex structure increases as the $t / C$ ratio increases. However, low $t / C$ ratio wings have three-dimensional surface separations at significantly higher angle of attack compared to high $t / C$ ratio wings.

- The results of force measurements are quite in line with the results of velocity and pressure measurements. At low angles of attack, higher lift coefficients, $C_{L}$, are achieved as the $t / C$ ratio increases. However, maximum $C_{L}$ values that can be reached are higher and appear at higher angle of attack as the $t / C$ ratio decreases. This indicates that the wing gets 
more resistive to the stall condition with decrease in $t / C$ ratio. Considering the drag coefficient, $C_{D}$, and lift-to-drag ratio, $C_{L} / C_{D}$, increase in $t / C$ ratio induces significant increase in $C_{D}$ and significant drop in $C_{L} / C_{D}$ values.

To conclude, due to the high dependency of aerodynamic performance and flow structure of non-slender delta wing on thickness-to-chord $t / C$ ratio, wing thickness might be considered to be utilized as a flow control parameter.

\subsection{Recommendations for Future Work}

In this thesis, the effect of thickness-to-chord ratio on flow structure and aerodynamics of a non-slender delta wing has been studied experimentally. The present investigation can be further improved in the following ways:

- Further investigation of Reynolds number role on $t / C$ ratio effect both in flow structure and aerodynamics by conducting experiments at higher Reynolds numbers.

- Using delta wing models with different leading edge bevel types to derive a solid conclusion about of $t / C$ ratio effect and possibly answer the different trends observed in the literature.

- Implementation of variable thickness wing models along with using airfoil sections to further investigate the applicability of utilization of $t / C$ ratio variation as a flow control technique. 


\section{REFERENCES}

[1] I. Gursul, "Vortex Flows on UAVs: Issues and Challenges'," vol. 108, no. 1090, pp. 597-610, 2004.

[2] I. Gursul, Z. Wang, and E. Vardaki, "Review of flow control mechanisms of leading-edge vortices," Prog. Aerosp. Sci., vol. 43, no. 7-8, pp. 246-270, 2007.

[3] P. B. Earnshaw, "An Experimental Investigation of the Structure of a Leading Edge Vortex," no. 3281, p. 11, 1962.

[4] D. L. Kohlman and W. H. Wentz, JR., "Vortex breakdown on slender sharpedged wings," J. Aircr., vol. 8, no. 3, pp. 156-161, Mar. 1971.

[5] M. M. Yavuz, M. Elkhoury, and D. Rockwell, "Near-Surface Topology and Flow Structure on a Delta Wing," AIAA J., vol. 42, no. 2, pp. 332-340, 2004.

[6] R. E. Gordnier and M. R. Visbal, "Compact Difference Scheme Applied to Simulation of Low-Sweep Delta Wing Flow.," AIAA J., vol. 43, no. 8, pp. 1744-1752, 2005.

[7] I. Gursul, R. Gordnier, and M. Visbal, "Unsteady aerodynamics of nonslender delta wings," Prog. Aerosp. Sci., vol. 41, no. 7, pp. 515-557, Oct. 2005.

[8] B. Gülsaçan, G. Şencan, and M. M. Yavuz, "Effect of Thickness-to-Chord Ratio on Flow Structure of a Low Swept Delta Wing," AIAA J., vol. 56, no. 12, pp. 4657-4668, 2018.

[9] H. Kawazoe, Y. Nakamura, T. Ono, and Y. Ushimaru, "Static and Total Pressure Distributions around a Thick Delta Wing with Rounded Leading Edge," AlAA 94-2321, 25th AlAA Plasmadynamics and Lasers Conference. 1994. 
[10] C. Breitsamter, "Unsteady flow phenomena associated with leading-edge vortices," Prog. Aerosp. Sci., vol. 44, no. 1, pp. 48-65, 2008.

[11] M. M. Yavuz and D. Rockwell, "Control of Flow Structure on Delta Wing with Steady Trailing-Edge Blow," AIAA J., vol. 44, no. 3, pp. 493-501, 2006.

[12] D. Hummel, "Effects of Boundary Layer Formation on the Vortical Flow above Slender Delta Wings," no. October, pp. 4-7, 2004.

[13] P. B. Earnshaw and J. a Lawford, "Low-Speed Wind-Tunnel Experiments on a Series of Sharp-Edged Delta Wings," Aeronautical, no. 3424, 1964.

[14] R. E. Gordnier and M. R. Visbal, "Unsteady vortex structure over a delta wing," J. Aircr., vol. 31, no. 1, pp. 243-248, 1994.

[15] M. Visbal and R. Gordnier, "On the Structure of the Shear Layer Emanating from a Swept Leading Edge at Angle of Attack," in 33rd AIAA Fluid Dynamics Conference and Exhibit, 2003.

[16] M. Gad-El-Hak and R. F. Blackwelder, "The discrete vortices from a delta wing," AIAA J., vol. 23, no. 6, pp. 961-962, 1985.

[17] R. Nelson and A. Pelletier, "The unsteady aerodynamics of slender wings and aircraft undergoing large amplitude maneuvers," Prog. Aerosp. Sci., 2003.

[18] E. C. Polhamus, "Predictions of vortex-lift characteristics by a leading-edge suction analogy,” J. Aircr., vol. 8, no. 4, pp. 193-199, 1971.

[19] M. V. Ol and M. Gharib, "Leading-Edge Vortex Structure of Nonslender Delta Wings at Low Reynolds Number," AIAA J., vol. 41, no. 1, pp. 16-26, 2003.

[20] H. Werlé, "Quelques résultats expérimentaux sur les ailes en flèche, aux faibles vitesses, obtenus en tunnel hydrodynamique," $\mathrm{La}$ Rech. Aéronautique, 1954. 
[21] G. S. Taylor, T. Schnorbus, and I. Gursul, “An Investigation of Vortex Flows Over Low Sweep Delta Wings," 33rd AIAA Fluid Dyn. Conf. Exhib., no. June, pp. 1-13, 2003.

[22] S. A. Woodiga and T. Liu, "Skin friction fields on delta wings," Exp. Fluids, vol. 47, no. 6, pp. 897-911, 2009.

[23] M. Zharfa, I. Ozturk, and M. M. Yavuz, "Flow Structure on Nonslender Delta Wing: Reynolds Number Dependence and Flow Control," AIAA J., vol. 54, no. 3, pp. 880-897, Mar. 2016.

[24] G. S. Taylor and I. Gursul, "Buffeting Flows over a Low-Sweep Delta Wing," AIAA J., vol. 42, no. 9, pp. 1737-1745, 2004.

[25] J. M. Delery, “Aspects of vortex breakdown,” Prog. Aerosp. Sci., vol. 30, no. 1, pp. 1-59, 1994.

[26] O. Lucca-Negro and T. O’Doherty, "Vortex breakdown: A review," Prog. Energy Combust. Sci., vol. 27, no. 4, pp. 431-481, 2001.

[27] T. Sarpkaya, "Effect of the Adverse Pressure Gradient on Vortex Breakdown," AIAA J., vol. 12, no. 5, pp. 602-607, 1974.

[28] T. Sarpkaya, "Vortex Breakdown in Swirling Conical Flows," AIAA J., vol. 9, no. 9, pp. 1792-1799, 1971.

[29] F. M. Payne, T. T. Ng, R. C. Nelson, and L. B. Schiff, "Visualization and Wake Surveys of Vortical Flow over a Delta Wing," AIAA J., vol. 26, no. 2, pp. 137-143, 1988.

[30] I. Gursul, "Unsteady Flow Phenomena Over Delta Wings at High Angle of Attack," AIAA J., vol. 32, no. 2, pp. 225-231, 1994.

[31] N. J. Wood and L. Roberts, "Control of vortical lift on delta wings by tangential leading-edge blowing," J. Aircr., vol. 25, no. 3, pp. 236-243, 1988. 
[32] N. J. Wood, L. Roberts, and Z. Celik, "Control of asymmetric vortical flows over delta wings at high anglesof attack," J. Aircr., vol. 27, no. 5, pp. 429_ $435,1990$.

[33] W. Gu, O. Robinson, and D. Rockwellf, "Control of vortices on a delta wing by leading-edge injection," AIAA J., vol. 31, no. 7, pp. 1177-1186, 1993.

[34] H. E. Helin and C. W. Watry, "Effects of Trailing-Edge Jet Entrainment on Delta Wing Vortices," AIAA J., vol. 32, no. 4, pp. 802-804, 1994.

[35] S. Phillips, C. Lambert, and I. Gursul, "Effect of a Trailing-Edge Jet on Fin Buffeting Introduction,” J. Aircaft, vol. 40, no. 3, pp. 590-599, 2003.

[36] P. Jiang, Z. Wang, and I. Gursul, "Effects of Unsteady Trailing-Edge Blowing on Delta Wing Aerodynamics," J. Aircr., vol. 47, no. 2, pp. 591$602,2010$.

[37] C. Shih and Z. Ding, "Trailing-Edge Jet Control of Leading-Edge Vortices of a Delta Wing," AIAA Journal, vol. 34, no. 7. pp. 1447-1457, 1996.

[38] Z. Wang, P. Jiang, and I. Gursul, "Effect of Thrust-Vectoring Jets on Delta Wing Aerodynamics," J. Aircr., vol. 44, no. 6, pp. 1877-1888, 2007.

[39] S. McCormick and I. Gursul, "Effect of shear-layer control on leading-edge vortices," J. Aircr., vol. 33, no. 6, pp. 1087-1093, 1996.

[40] C.-H. Kuo and N.-Y. Lu, "Unsteady Vortex Structure over Delta-Wing Subject to Transient Along-Core Blowing," AIAA J., vol. 36, no. 9, pp. 1658 1664, 1998.

[41] S. Guillot, E. Gutmark, and T. Garrison, "Delay of vortex breakdown over a delta wing via near-core blowing," AIAA Pap., 1998.

[42] Y. Liu et al., "Reactive Flow Control of Delta-Wing Vortex," J. Aircr., vol. 45 , no. 3, pp. 880-892, 2008.

[43] A. M. Mitchell, D. Barberis, P. Molton, J. Dé, and lery, "Oscillation of 
Vortex Breakdown Location and Blowing Control of Time-Averaged Location," AIAA J., vol. 38, no. 5, pp. 793-803, 2000.

[44] A. Kölzsch and C. Breitsamter, "Vortex-Flow Manipulation on a Generic Delta-Wing Configuration,” J. Aircr., vol. 51, no. 5, pp. 1380-1390, 2014.

[45] C. Çetin, A. Çelik, and M. M. Yavuz, "Control of Flow Structure over a Nonslender Delta Wing Using Periodic Blowing," AIAA J., vol. 56, no. 1, pp. 90-99, 2018.

[46] Y. Guy, J. Morrow, and T. McLaughlin, "Control of vortex breakdown on a delta wing by periodic blowing and suction," in 37th Aerospace Sciences Meeting and Exhibit, 1999.

[47] Y. Guy, J. Morrow, T. McLaughlin, and I. Wygnanski, "Pressure Measurements and Flow Field Visualization on a Delta Wing with Periodic Blowing and Suction," AIAA Pap., no. c, 1999.

[48] S. A. Morton, Y. Guy, J. A. Morrow, and D. C. Blake, "AIAA 99-3195 Numerical Simulation of Periodic Suction and Blowing Control of Vortex Breakdown on a Delta Wing 17th Applied Aerodynamics Conference 28 June - 1 July, 1999 / Norfolk, VA," Control, no. c, 1999.

[49] A. Çelik, C. Çetin, and M. M. Yavuz, "Effect of Passive Bleeding on Flow Structure over a Nonslender Delta Wing," AIAA J., vol. 55, no. 8, pp. 1-11, 2017.

[50] I. Gursul and G. Batta, "Active control of vortex breakdown over a delta wing," AIAA J., vol. 33, no. 9, pp. 1743-1745, 1995.

[51] Y. Liu, L. X. Zuo, and J. J. Wang, "Effects of flexibility on aerodynamic performance of delta wings with different sweep angles," Sci. China Physics, Mech. Astron., vol. 53, no. 5, pp. 915-922, 2010.

[52] G. Taylor, Z. Wang, E. Vardaki, and I. Gursul, "Lift Enhancement over Flexible Nonslender Delta Wings," AIAA J., vol. 45, no. 12, pp. 2979-2993, 
2007.

[53] T. Goruney and D. Rockwell, "Flow past a delta wing with a sinusoidal leading edge: near-surface topology and flow structure," Exp. Fluids, vol. 47, no. 2, pp. 321-331, Aug. 2009.

[54] H. Chen and J.-J. Wang, "Vortex structures for flow over a delta wing with sinusoidal leading edge," Exp. Fluids, vol. 55, no. 6, p. 1761, Jun. 2014.

[55] H. Chen, C. Pan, and J. Wang, "Effects of sinusoidal leading edge on delta wing performance and mechanism," Sci. China Technol. Sci., vol. 56, no. 3, pp. 772-779, 2013.

[56] A. Çelik and M. M. Yavuz, "Effect of Edge Modifications on Flow Structure of Low Swept Delta Wing,” AIAA J., vol. 54, no. 5, pp. 1789-1797, 2016.

[57] J. J. Wang and S. F. Lu, "Effects of leading-edge bevel angle on the aerodynamic forces of a non-slender 50?? delta wing," Aeronaut. J., vol. 109, no. 1098 , pp. 403-407, 2005 .

[58] D. Ma, Y. Zhao, Y. Qiao, and G. Li, "Effects of relative thickness on aerodynamic characteristics of airfoil at a low Reynolds number," Chinese J. Aeronaut., vol. 28, no. 4, pp. 1003-1015, 2015.

[59] A. Sharma and M. Visbal, "Numerical Investigation of the Effect of Airfoil Thickness on Onset of Dynamic Stall,”pp. 1-31, 2017.

[60] J.-H. Ran, Z.-Q. Liu, and P. Bai, "The effect of relative thickness to the dynamic aerodynamic characteristics about pitching airfoils," Kongqi Donglixue Xuebao/Acta Aerodyn. Sin., vol. 26, no. 2, p. 2018, 2008.

[61] R. D. Witcofski and D. C. Marcum, Jr., Effect of Thickness and Sweep Angle on the Longitudinal Aerodynamic Characteristics of Slab Delta Planforms at a Mach Number of 20, no. June 1966. 1966.

[62] S. Y. Ruo, E. Carson Yates, and J. G. Theisen, "Calculation of Unsteady Transonic Aerodynamics for Oscillating Wings with Thickness," vol. 11, no. 
10, pp. 601-608, 1975.

[63] M. V Lowson and A. J. C. Ponton, "Symmetry Breaking in Vortex Flows on Conical Bodies," AIAA Journal, vol. 30, no. 6. p. 797, 1992.

[64] A. Parker, "Aerodynamic characteristics of slender wings with sharp leading edges- A review," J. Aircr., 1976.

[65] M. V. Lowson and A. J. Riley, "Vortex Breakdown Control by Delta-Wing Geometry," Journal of Aircraft, vol. 32, no. 4. pp. 832-838, 1995.

[66] S. A. Thompson, "The Unsteady Aerodynamics of a Delta Wing Undergoing large Amplitude Pitching Motion," PhD Dissertation, University of Notre Dome. 1992.

[67] J. Saltzman and T. G. Ayers, "Review of Flight-to-Wind-Tunnel Drag Correlation Edwin," Journal of Aircraft, vol. 19, no. 10. pp. 801-811, 1982.

[68] H. Golparvar, S. Irani, and M. Mousavi Sani, "Experimental investigation of linear and nonlinear aeroelastic behavior of a cropped delta wing with store in low subsonic flow," J. Brazilian Soc. Mech. Sci. Eng., vol. 38, no. 4, pp. 1113-1130, 2016.

[69] A. McClain, Z.-J. Wang, E. Vardaki, and I. Gursul, "Unsteady aerodynamics of free-to-roll non-slender delta wings," 45th AIAA Aerospace Sciences Meeting and Exhibit, Reno, NV, AIAA Paper, vol. 1074. pp. 1-14, 2007.

[70] Kline and S. J., "Describing Uncertainty in Single Sample Experiments," Mech. Eng., vol. 75, pp. 3-8, 1953.

[71] INSIGHT 4G TM GLOBAL IMAGE, ACQUISITION , ANALYSIS , \& DISPLAY SOFTWARE. 2015. 


\section{APPENDIX A}

\section{A. CODE FOR CALCULATING RMS VALUES OF VELOCITY COMPONENTS IN TECPLOT FOCUS.}

$\% \% \% \mathrm{~V}$ is the normal to surface velocity component, for rms values of transverse $\% \% \%$ velocity component replace $\mathrm{V}$ with $\mathrm{U}$.

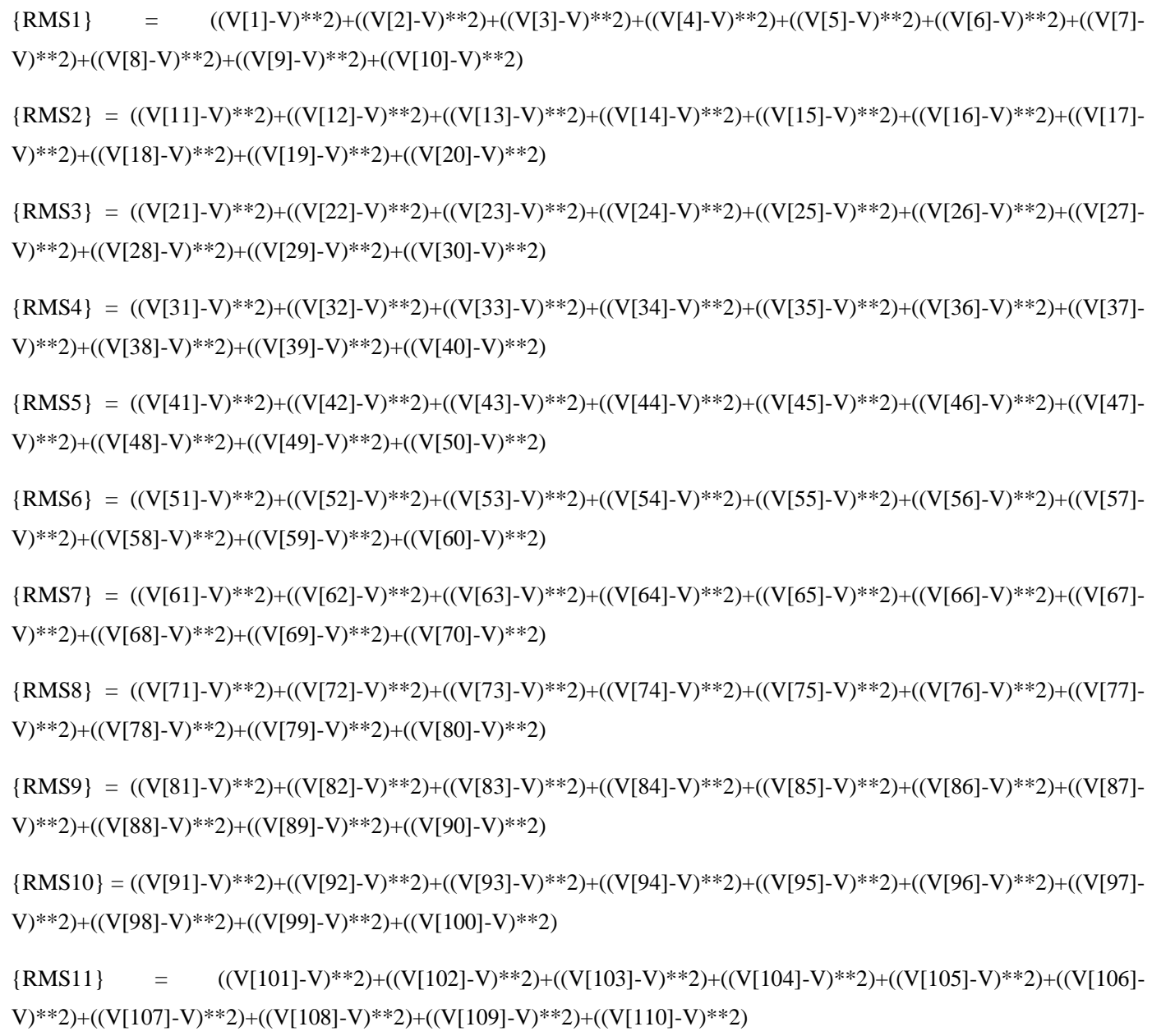


$\{$ RMS12 $\}=\left((\mathrm{V}[111]-\mathrm{V})^{* * 2}\right)+((\mathrm{V}[112]-\mathrm{V}) * * 2)+((\mathrm{V}[113]-\mathrm{V}) * * 2)+((\mathrm{V}[114]-\mathrm{V}) * * 2)+((\mathrm{V}[115]-\mathrm{V}) * * 2)+((\mathrm{V}[116]-$ $\left.\mathrm{V})^{* * 2}\right)+\left((\mathrm{V}[117]-\mathrm{V})^{* * 2}\right)+\left((\mathrm{V}[118]-\mathrm{V})^{* * 2}\right)+\left((\mathrm{V}[119]-\mathrm{V})^{* * 2}\right)+\left((\mathrm{V}[120]-\mathrm{V})^{* * 2}\right)$

$\{$ RMS13 $\}=\left((\mathrm{V}[121]-\mathrm{V})^{* * 2}\right)+((\mathrm{V}[122]-\mathrm{V}) * * 2)+((\mathrm{V}[123]-\mathrm{V}) * * 2)+((\mathrm{V}[124]-\mathrm{V}) * * 2)+((\mathrm{V}[125]-\mathrm{V}) * * 2)+((\mathrm{V}[126]-$ $\left.\mathrm{V})^{* * 2}\right)+((\mathrm{V}[127]-\mathrm{V}) * * 2)+((\mathrm{V}[128]-\mathrm{V}) * * 2)+((\mathrm{V}[129]-\mathrm{V}) * * 2)+((\mathrm{V}[130]-\mathrm{V}) * * 2)$

$\{$ RMS14 $\}=\left((\mathrm{V}[131]-\mathrm{V})^{* * 2}\right)+\left((\mathrm{V}[132]-\mathrm{V})^{* * 2}\right)+\left((\mathrm{V}[133]-\mathrm{V})^{* * 2}\right)+\left((\mathrm{V}[134]-\mathrm{V})^{* * 2}\right)+\left((\mathrm{V}[135]-\mathrm{V})^{* * 2}\right)+((\mathrm{V}[136]-$ $\left.\mathrm{V})^{* * 2}\right)+((\mathrm{V}[137]-\mathrm{V}) * * 2)+((\mathrm{V}[138]-\mathrm{V}) * * 2)+((\mathrm{V}[139]-\mathrm{V}) * * 2)+((\mathrm{V}[140]-\mathrm{V}) * * 2)$

$\{\mathrm{RMS15}\}=\left((\mathrm{V}[141]-\mathrm{V})^{* * 2}\right)+\left((\mathrm{V}[142]-\mathrm{V})^{* * 2}\right)+\left((\mathrm{V}[143]-\mathrm{V})^{* * 2}\right)+\left((\mathrm{V}[144]-\mathrm{V})^{* * 2}\right)+\left((\mathrm{V}[145]-\mathrm{V})^{* * 2}\right)+((\mathrm{V}[146]-$ $\mathrm{V}) * * 2)+((\mathrm{V}[147]-\mathrm{V}) * * 2)+\left((\mathrm{V}[148]-\mathrm{V})^{* * 2}\right)+((\mathrm{V}[149]-\mathrm{V}) * * 2)+((\mathrm{V}[150]-\mathrm{V}) * * 2)$

$\{\mathrm{RMS16}\}=((\mathrm{V}[151]-\mathrm{V}) * * 2)+((\mathrm{V}[152]-\mathrm{V}) * * 2)+\left((\mathrm{V}[153]-\mathrm{V})^{* * 2}\right)+((\mathrm{V}[154]-\mathrm{V}) * * 2)+((\mathrm{V}[155]-\mathrm{V}) * * 2)+((\mathrm{V}[156]-$ $\left.\mathrm{V})^{* * 2}\right)+((\mathrm{V}[157]-\mathrm{V}) * * 2)+((\mathrm{V}[158]-\mathrm{V}) * * 2)+((\mathrm{V}[159]-\mathrm{V}) * * 2)+((\mathrm{V}[160]-\mathrm{V}) * * 2)$

$\{$ RMS17 $\}=((\mathrm{V}[161]-\mathrm{V}) * * 2)+\left((\mathrm{V}[162]-\mathrm{V})^{* * 2}\right)+\left((\mathrm{V}[163]-\mathrm{V})^{* * 2}\right)+\left((\mathrm{V}[164]-\mathrm{V})^{* * 2}\right)+\left((\mathrm{V}[165]-\mathrm{V})^{* * 2}\right)+((\mathrm{V}[166]-$ $\mathrm{V}) * * 2)+((\mathrm{V}[167]-\mathrm{V}) * * 2)+((\mathrm{V}[168]-\mathrm{V}) * * 2)+((\mathrm{V}[169]-\mathrm{V}) * * 2)+((\mathrm{V}[170]-\mathrm{V}) * * 2)$

$\{\mathrm{RMS18}\}=((\mathrm{V}[171]-\mathrm{V}) * * 2)+\left((\mathrm{V}[172]-\mathrm{V})^{* * 2}\right)+\left((\mathrm{V}[173]-\mathrm{V})^{* * 2}\right)+\left((\mathrm{V}[174]-\mathrm{V})^{* * 2}\right)+\left((\mathrm{V}[175]-\mathrm{V})^{* * 2}\right)+((\mathrm{V}[176]-$ $\left.\mathrm{V})^{* * 2}\right)+\left((\mathrm{V}[177]-\mathrm{V})^{* *} 2\right)+\left((\mathrm{V}[178]-\mathrm{V})^{* * 2}\right)+\left((\mathrm{V}[179]-\mathrm{V})^{* * 2}\right)+\left((\mathrm{V}[180]-\mathrm{V})^{* * 2}\right)$

$\{\mathrm{RMS19}\}=((\mathrm{V}[181]-\mathrm{V}) * * 2)+((\mathrm{V}[182]-\mathrm{V}) * * 2)+((\mathrm{V}[183]-\mathrm{V}) * * 2)+((\mathrm{V}[184]-\mathrm{V}) * * 2)+((\mathrm{V}[185]-\mathrm{V}) * * 2)+((\mathrm{V}[186]-$ $\left.\mathrm{V})^{* * 2}\right)+((\mathrm{V}[187]-\mathrm{V}) * * 2)+((\mathrm{V}[188]-\mathrm{V}) * * 2)+((\mathrm{V}[189]-\mathrm{V}) * * 2)+((\mathrm{V}[190]-\mathrm{V}) * * 2)$

$\{$ RMS20 $\}=((\mathrm{V}[191]-\mathrm{V}) * * 2)+((\mathrm{V}[192]-\mathrm{V}) * * 2)+((\mathrm{V}[193]-\mathrm{V}) * * 2)+((\mathrm{V}[194]-\mathrm{V}) * * 2)+((\mathrm{V}[195]-\mathrm{V}) * * 2)+((\mathrm{V}[196]-$ $\left.\mathrm{V})^{* * 2}\right)+((\mathrm{V}[197]-\mathrm{V}) * * 2)+((\mathrm{V}[198]-\mathrm{V}) * * 2)+((\mathrm{V}[199]-\mathrm{V}) * * 2)+((\mathrm{V}[200]-\mathrm{V}) * * 2)$

$\{$ VRMS $\}=\operatorname{sqrt}((\{$ RMS 1$\}+\{$ RMS 2$\}+\{$ RMS3 $\}+\{$ RMS4 $\}+\{$ RMS5 $\}+\{$ RMS6 $\}+\{$ RMS 7$\}+\{$ RMS 8$\}+\{$ RMS9 $\}+\{$ RMS10 $\}+\{$ R MS11 $\}+\{$ RMS12 $\}+\{$ RMS13 $\}+\{$ RMS14 $\}+\{$ RMS15 $\}+\{$ RMS16 $\}+\{$ RMS17 $\}+\{$ RMS18 $\}+\{$ RMS19 $\}+\{$ RMS20 $\}) / 200) / 3.52$ 


\section{APPENDIX B}

\section{B. SOURCE CODES FOR PRESSURE COEFFICIENT UNCERTAINITY CALCULATION}

clear all

clc

$\%=======$ Uncertainty Calculation=================

P_W $=-31.31128721 ; \quad \% \% \mathrm{~Pa}$ (Static Pressure on the Wing)

$\mathrm{P} \_$inf $=-22.87358627 ; \quad \% \% \mathrm{~Pa}($ Static Pressure of the Free Stream)

P_stag=-10.81869585; \%\% $\quad \%$ (Stagnation Pressure of the Free Stream

$\mathrm{P} \_\mathrm{r}=0.075 ; \quad \% \% \mathrm{~Pa}$ (Measurement Resolution of the Device $(0.003 \%$ FS $)$ )

Span $=0.3 ; \quad \% \% \mathrm{~m}$ (Wing Span, Measured with ruler)

Chord $=0.105 ; \quad \% \% \mathrm{~m}$ (Wing Chord, Measured with rules)

d_rul $=0.001 \quad \% \% \mathrm{~m}$ (Resolution of ruler)

As $=0.5 * \operatorname{Span}^{*}$ Chord $\% \% \mathrm{~m}^{\wedge} 2($ Wing Surface Area)

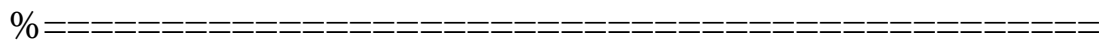

$\%$ Uncertainty of Dynamic Pressure

P_dyn=P_stag-P_inf;

dPdyn_Pstag $=1$;

dPdyn_Pinf=-1;

dPdyn $=\left(\left(d P d y n \_P s t a g * P \_r\right)^{\wedge} 2+\left(d P d y n \_P i n f * P \_r\right)^{\wedge} 2\right)^{\wedge} 0.5$

Urel_dPdyn=dPdyn/P_dyn \% (Relative uncertainty of Dynamic Pressure)

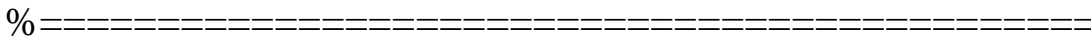

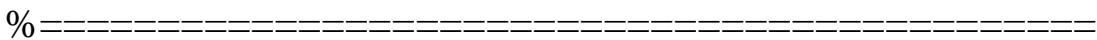

$\%$ Uncertainty of Wing Surface Area

dAs_sp $=0.5^{*}$ Chord; 
dAs_ch $=0.5 *$ Span;

$\mathrm{dAs}=\left(\left(\mathrm{dAs} \_s p^{*} \mathrm{~d} \_ \text {rul }\right)^{\wedge} 2+\left(\mathrm{dAs} \_ \text {ch*d_rul }\right)^{\wedge} 2\right)^{\wedge} 0.5$;

Urel_dAs=dAs/As $\%$ (Relative uncertainty of Wing Surface Area)

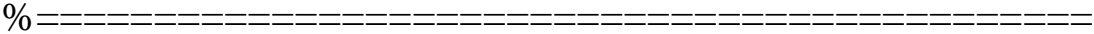

\%Uncertainty of Pressure Coefficient

$\mathrm{Cp}=\left(\mathrm{P} \_w-P \_\right.$inf $) /\left(\mathrm{P} \_\right.$stag-P_inf $) ;$

$\mathrm{dCp} \_\mathrm{Pw}=1 /\left(\mathrm{P} \_\right.$stag-P_inf $)$;

dCp_Pinf $=\left(P \_w-P \_s t a g\right) /\left(P \_ \text {inf-P_stag }\right)^{\wedge} 2$;

dCp_Pstag $=\left(P \_\right.$inf-P_w $) /\left(P \_ \text {inf-P_stag }\right)^{\wedge} 2$;

$\mathrm{dCp}=\left(\left(\mathrm{dCp} \_P w^{*} P \_r\right)^{\wedge} 2+\left(\mathrm{dCp} \_P i n f * P \_r\right)^{\wedge} 2+\left(\mathrm{dCp} \_P s t a g * P \_r\right)^{\wedge} 2\right)^{\wedge} 0.5$

Urel_Cp=dCp/-Cp \% (Relative uncertainty of Pressure Coefficient)

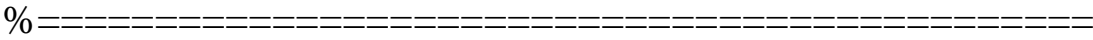




\section{APPENDIX C}

\section{FORMULAS FOR LIFT AND DRAG COEFFICIENTS AND} CORROSPONDING UNCERTAINITY CALCULATIONS.

$$
\begin{aligned}
& \mathrm{N}=\left(\mathrm{M}_{2}-\mathrm{M}_{1}\right) / \mathrm{L}_{1} \\
& A=\left(M_{3}-M_{2}-N_{2}\right) / L_{3} \\
& \text { Lift }=\mathrm{N} \cos \alpha-\mathrm{A} \sin \alpha \\
& \text { Drag }=\mathrm{N} \sin \alpha+\mathrm{A} \cos \alpha \\
& \text { Lift }_{\text {force }}=\frac{\text { Lift }_{\text {strain }}}{\mathrm{X}} \\
& \operatorname{Drag}_{\text {force }}=\frac{\text { Drag }_{\text {strain }}}{X} \\
& \mathrm{C}_{\mathrm{L}}=\frac{\text { Lift }_{\text {force }}}{\left(0.5 \rho \mathrm{V}^{2} A_{\text {Ref }}\right)}=\frac{\text { Lift }_{\text {force }}}{\left(0.5 \rho \mathrm{V}^{2} \mathrm{C}^{2}\right)} \\
& \mathrm{C}_{\mathrm{D}}=\frac{\text { Drag }_{\text {force }}}{\left(0.5 \rho \mathrm{V}^{2} A_{\text {Ref }}\right)}=\frac{\text { Drag }_{\text {force }}}{\left(0.5 \rho \mathrm{V}^{2} \mathrm{C}^{2}\right)} \\
& \omega_{\mathrm{N}}=\left(\left(\frac{\omega_{\mathrm{M}_{2}}}{\mathrm{~L}_{1}}\right)^{2}+\left(-\frac{\omega_{\mathrm{M}_{1}}}{\mathrm{~L}_{1}}\right)^{2}+\left(\frac{\omega_{\mathrm{L}_{1}}\left(-\left(\mathrm{M}_{2}-\mathrm{M}_{1}\right)\right)}{\mathrm{L}_{1}^{2}}\right)^{2}\right)^{0.5} \\
& \omega_{\mathrm{A}}=\left(\frac{\left(\omega_{\mathrm{M}_{3}}^{2}+\omega_{\mathrm{M}_{2}}^{2}\right)}{\mathrm{L}_{3}^{2}}+\left(-\frac{\omega_{\mathrm{N}} * \mathrm{~L}_{2}}{\mathrm{~L}_{3}}\right)^{2}+\left(-\frac{\omega_{\mathrm{L}_{2}} * \mathrm{~N}}{\mathrm{~L}_{3}}\right)^{2}+\left(\frac{\omega_{\mathrm{L}_{3}} * \mathrm{~N} * \mathrm{~L}_{2}}{\mathrm{~L}_{3}^{2}}\right)^{2}\right)^{0.5} \\
& \omega_{\text {Lift }_{\text {strain }}}=\left(\left(\omega_{\mathrm{N}} * \cos \alpha\right)^{2}+\left(-\omega_{\mathrm{A}} \sin \alpha\right)^{2}+\left(\omega_{\alpha}(-\mathrm{N} \sin \alpha-\mathrm{A} \cos \alpha)\right)^{2}\right)^{0.5} \\
& \omega_{\text {Lift }_{\text {force }}}=\left(\left(\frac{\omega_{\text {lift }_{\text {strain }}}}{\mathrm{X}}\right)^{2}+\left(-\omega_{\mathrm{X}} * \frac{\text { Lift }_{\text {strain }}}{\mathrm{X}^{2}}\right)^{2}\right)^{0.5}
\end{aligned}
$$




$$
\begin{aligned}
& \omega_{\mathrm{C}_{\mathrm{L}}}=\left(\left(\frac{\omega_{\mathrm{Lift}_{\text {force }}}}{0.5 * \rho * \mathrm{~V}^{2} * \mathrm{C}^{2}}\right)^{2}+\left(\frac{-\omega_{\rho} * \mathrm{Lift}_{\text {force }}}{0.5 * \rho^{2} * \mathrm{~V}^{2} * \mathrm{C}^{2}}\right)^{2}+\left(\frac{-2 * \omega_{\mathrm{V}} * \mathrm{Lift}_{\text {force }}}{0.5 * \rho * \mathrm{~V}^{3} * \mathrm{C}^{2}}\right)^{2}\right. \\
& \left.+\left(\frac{-2 * \omega_{\mathrm{C}} * \mathrm{Lift}_{\text {force }}}{0.5 * \rho * \mathrm{~V}^{2} * \mathrm{C}^{3}}\right)^{2}\right)^{0.5} \\
& \omega_{\text {Drag }_{\text {strain }}}=\left(\left(\omega_{\mathrm{N}} * \sin \alpha\right)^{2}+\left(\omega_{\mathrm{A}} \cos \alpha\right)^{2}+\left(\omega_{\alpha}(\mathrm{N} \cos \alpha-\mathrm{A} \sin \alpha)\right)^{2}\right)^{0.5} \\
& \omega_{\text {Drag }_{\text {force }}}=\left(\left(\frac{\omega_{\text {Drag }_{\text {strain }}}}{\mathrm{X}}\right)^{2}+\left(-\omega_{\mathrm{X}} * \frac{\text { Drag }_{\text {strain }}}{\mathrm{X}^{2}}\right)^{2}\right)^{0.5} \\
& \omega_{\mathrm{C}_{\mathrm{D}}}=\left(\left(\frac{\omega_{\text {Drag }_{\text {force }}}}{0.5 * \rho * \mathrm{~V}^{2} * \mathrm{C}^{2}}\right)^{2}+\left(\frac{-\omega_{\rho} * \operatorname{Drag}_{\text {force }}}{0.5 * \rho^{2} * \mathrm{~V}^{2} * \mathrm{C}^{2}}\right)^{2}+\left(\frac{-2 * \omega_{\mathrm{V}} * \text { Drag }_{\text {force }}}{0.5 * \rho * \mathrm{~V}^{3} * \mathrm{C}^{2}}\right)^{2}\right. \\
& \left.+\left(\frac{-2 * \omega_{\mathrm{C}} * \operatorname{Drag}_{\text {force }}}{0.5 * \rho * \mathrm{~V}^{2} * \mathrm{C}^{3}}\right)^{2}\right)^{0.5} \\
& \mathrm{u}_{\mathrm{C}_{\mathrm{L}}}=\omega_{\mathrm{C}_{\mathrm{L}}} / \mathrm{C}_{\mathrm{L}} \\
& \mathrm{u}_{\mathrm{C}_{\mathrm{D}}}=\omega_{\mathrm{C}_{\mathrm{D}}} / \mathrm{C}_{\mathrm{D}}
\end{aligned}
$$

Table C.1 Corresponding uncertainty values of force measurements for wing with $t / C=15 \%$ at different angle of attacks.

\begin{tabular}{|l|c|c|c|c|c|c|c|c|c|}
\hline$\alpha^{\circ}$ & 0 & 4 & 12 & 14 & 18 & 20 & 24 & 26 & 30 \\
\hline$\omega_{\mathrm{M} 1}$ & $3 \mathrm{E}-07$ & $3 \mathrm{E}-07$ & $3 \mathrm{E}-07$ & $3 \mathrm{E}-07$ & $3 \mathrm{E}-07$ & $3 \mathrm{E}-07$ & $3 \mathrm{E}-07$ & $3 \mathrm{E}-07$ & $3 \mathrm{E}-07$ \\
\hline$\omega_{\mathrm{M} 2}$ & $3 \mathrm{E}-07$ & $3 \mathrm{E}-07$ & $3 \mathrm{E}-07$ & $3 \mathrm{E}-07$ & $3 \mathrm{E}-07$ & $3 \mathrm{E}-07$ & $3 \mathrm{E}-07$ & $3 \mathrm{E}-07$ & $3 \mathrm{E}-07$ \\
\hline$\omega_{\mathrm{M} 3}$ & $3 \mathrm{E}-07$ & $3 \mathrm{E}-07$ & $3 \mathrm{E}-07$ & $3 \mathrm{E}-07$ & $3 \mathrm{E}-07$ & $3 \mathrm{E}-07$ & $3 \mathrm{E}-07$ & $3 \mathrm{E}-07$ & $3 \mathrm{E}-07$ \\
\hline$\omega_{\mathrm{N}}$ & $4 \mathrm{E}-06$ & $4 \mathrm{E}-06$ & $4 \mathrm{E}-06$ & $4 \mathrm{E}-06$ & $4 \mathrm{E}-06$ & $4 \mathrm{E}-06$ & $4 \mathrm{E}-06$ & $4 \mathrm{E}-06$ & $4 \mathrm{E}-06$ \\
\hline$\omega_{\mathrm{A}}$ & $2 \mathrm{E}-06$ & $2 \mathrm{E}-06$ & $2 \mathrm{E}-06$ & $2 \mathrm{E}-06$ & $2 \mathrm{E}-06$ & $2 \mathrm{E}-06$ & $2 \mathrm{E}-06$ & $2 \mathrm{E}-06$ & $2 \mathrm{E}-06$ \\
\hline$\omega_{\text {lift-strain }}$ & $4 \mathrm{E}-06$ & $4 \mathrm{E}-06$ & $4 \mathrm{E}-06$ & $4 \mathrm{E}-06$ & $4 \mathrm{E}-06$ & $4 \mathrm{E}-06$ & $4 \mathrm{E}-06$ & $4 \mathrm{E}-06$ & $4 \mathrm{E}-06$ \\
\hline$\omega_{\text {lift-force }}$ & 0.0013 & 0.0027 & 0.0038 & 0.0038 & 0.004 & 0.0038 & 0.0032 & 0.0032 & 0.0035 \\
\hline$\omega_{\mathrm{CL}}$ & 0.0098 & 0.0215 & 0.0298 & 0.0304 & 0.0313 & 0.0299 & 0.0255 & 0.0249 & 0.028 \\
\hline$\omega_{\text {drag-strain }}$ & $2 \mathrm{E}-06$ & $2 \mathrm{E}-06$ & $2 \mathrm{E}-06$ & $2 \mathrm{E}-06$ & $2 \mathrm{E}-06$ & $2 \mathrm{E}-06$ & $2 \mathrm{E}-06$ & $3 \mathrm{E}-06$ & $3 \mathrm{E}-06$ \\
\hline$\omega_{\text {drag-force }}$ & 0.0024 & 0.0029 & 0.0045 & 0.0047 & 0.0052 & 0.0054 & 0.0054 & 0.0058 & 0.0065 \\
\hline$\omega_{\mathrm{CD}}$ & 0.0187 & 0.0232 & 0.0355 & 0.0374 & 0.0409 & 0.0425 & 0.0429 & 0.046 & 0.0514 \\
\hline$U_{\mathrm{CL}}$ & 0.0561 & 0.0554 & 0.0553 & 0.0552 & 0.0552 & 0.0552 & 0.0553 & 0.0553 & 0.0552 \\
\hline$U_{\mathrm{CD}}$ & 0.055 & 0.055 & 0.055 & 0.055 & 0.055 & 0.055 & 0.055 & 0.055 & 0.055 \\
\hline
\end{tabular}


APPENDIX D

D. LASER ILLUMINATED SURFACE FLOW SMOKE VISUALIZATIONS FOR THICKNESS-TO-CHORD RATIOS OF 0.033, $0.05,0.10,0.15$ FOR RE $=10000$ AT $\alpha=6^{\circ}, 10$ AND FOR RE=10000, AND 15000 AT $\alpha=14^{\circ}$. 

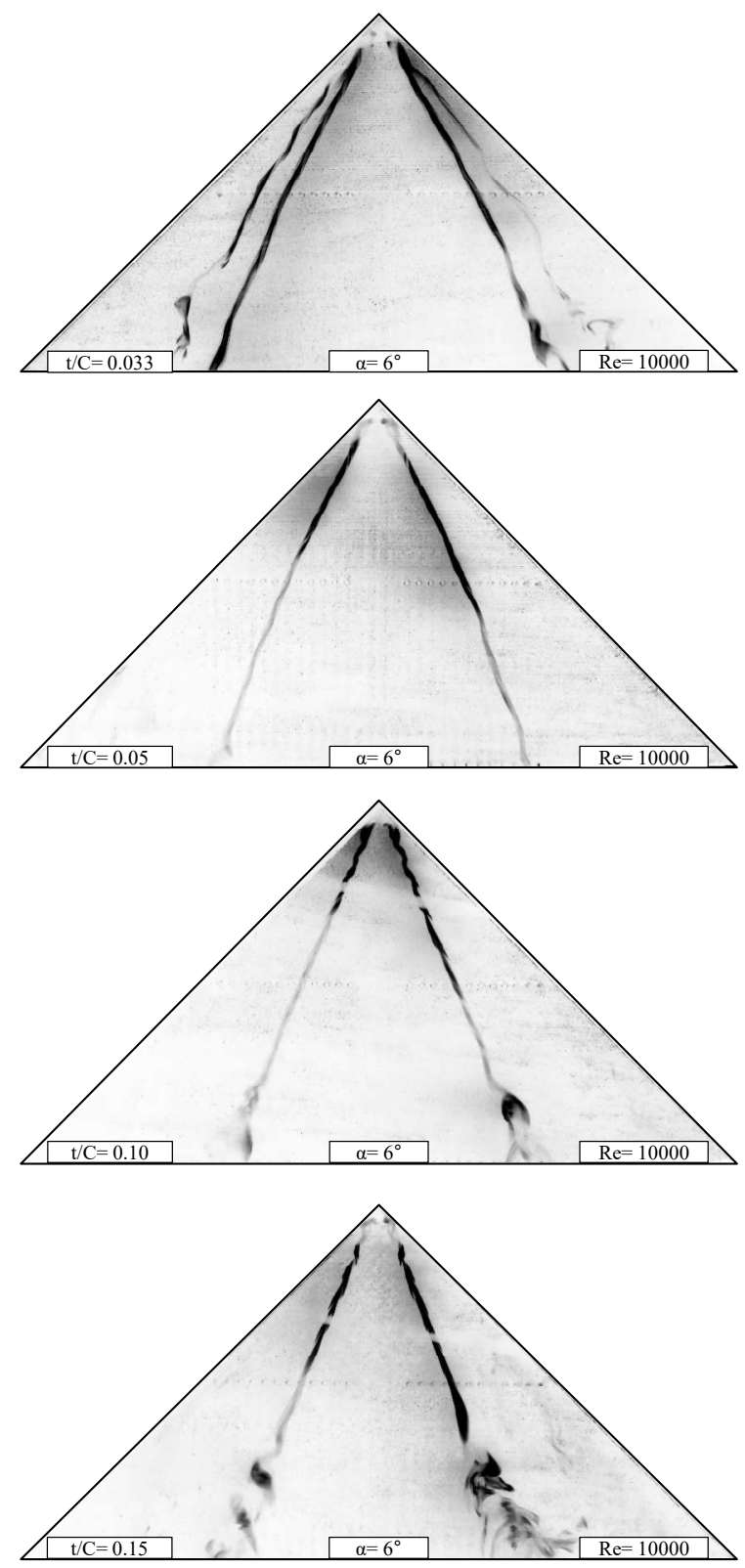

Figure D.1 Laser-illuminated surface flow smoke visualizations of $t / C=$ $0.033,0.05,0.10$ and 0.15 for $R e=1 \times 10^{4}$ at angle of attack of $\alpha=6 \mathrm{deg}$. 

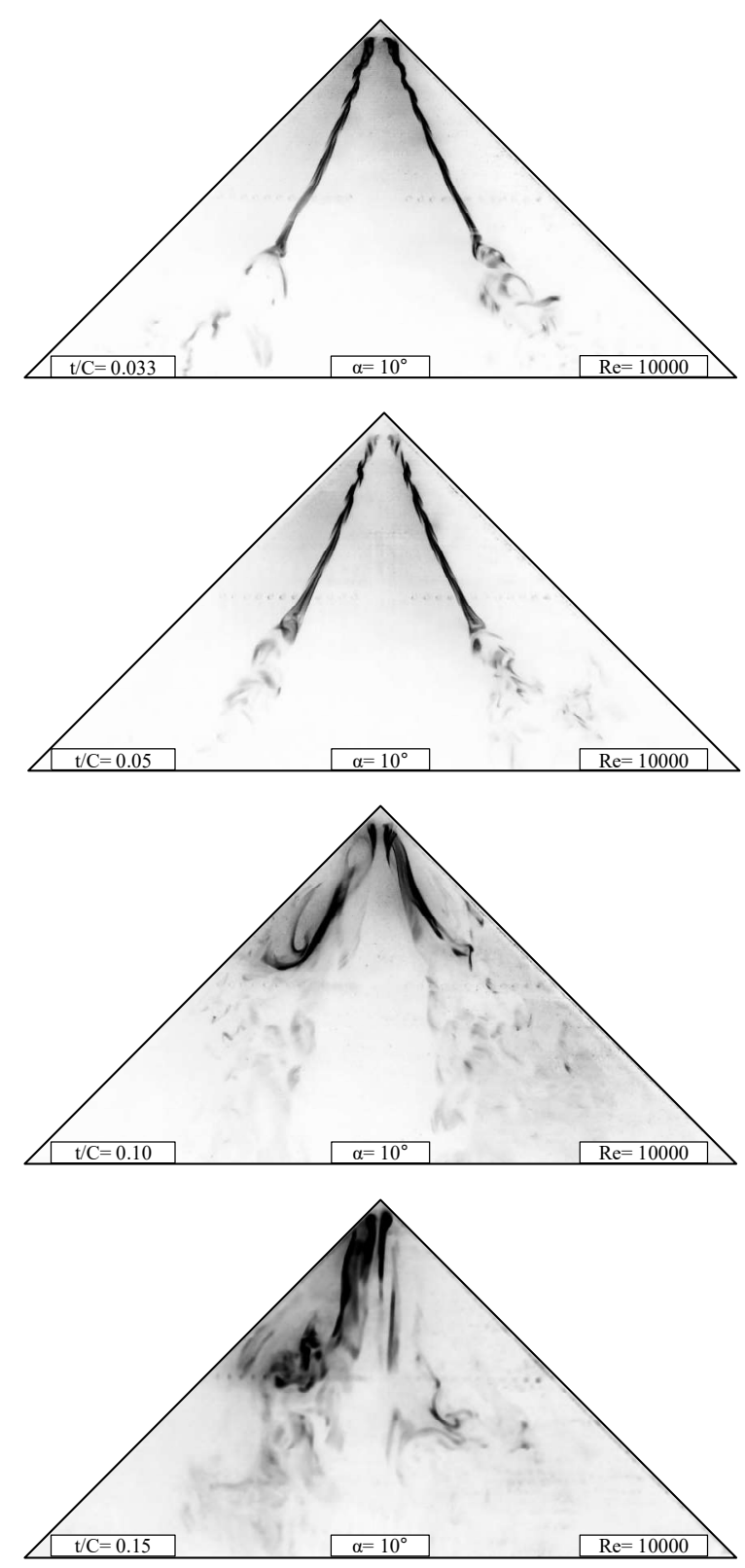

Figure D.2 Laser-illuminated surface flow smoke visualizations of $t / C=$ $0.033,0.05,0.10$ and 0.15 for $R e=1 \times 10^{4}$ at angle of attack of $\alpha=10 \mathrm{deg}$. 

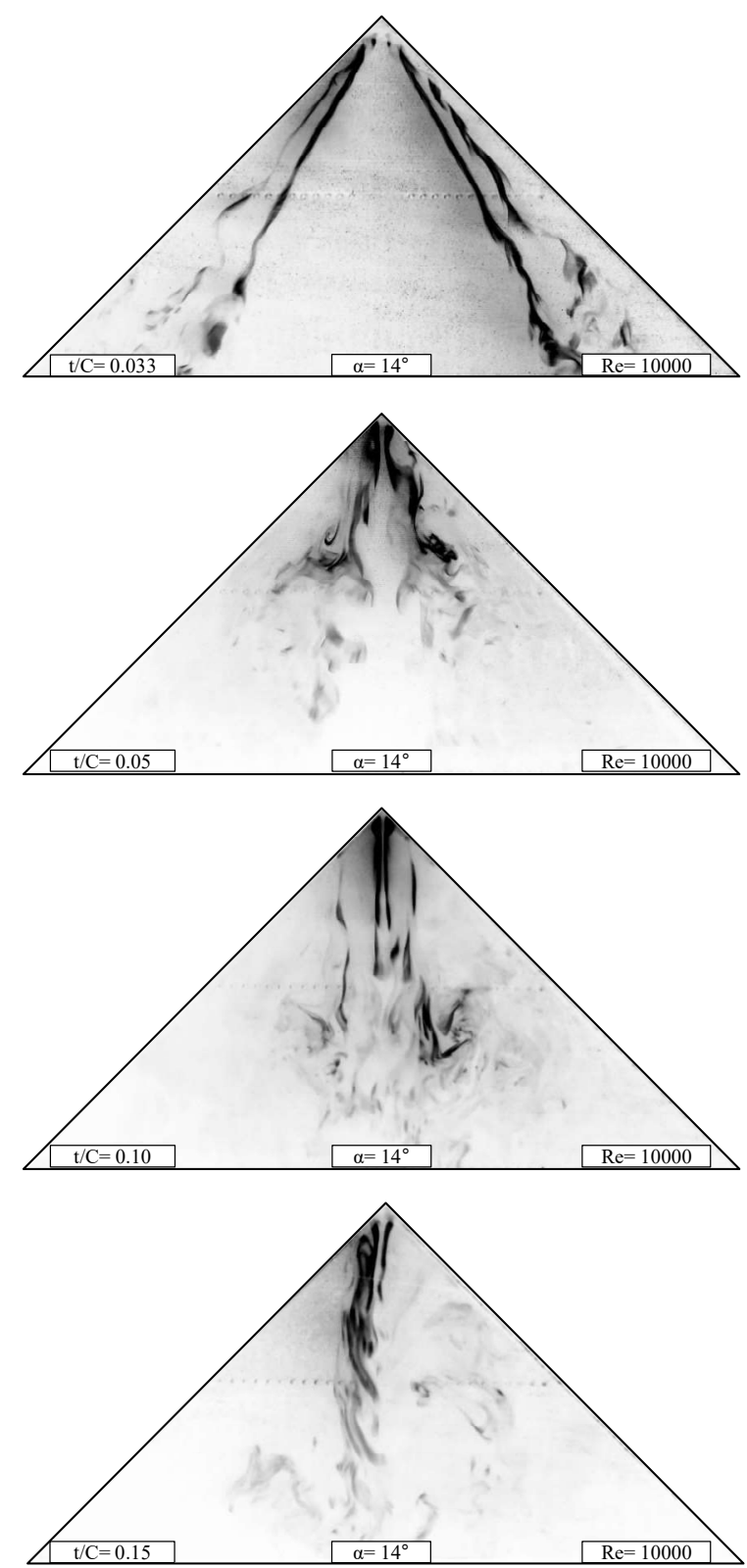

Figure D.3 Laser-illuminated surface flow smoke visualizations of $t / C=$ $0.033,0.05,0.10$ and 0.15 for $R e=1 \times 10^{4}$ at angle of attack of $\alpha=14 \mathrm{deg}$. 

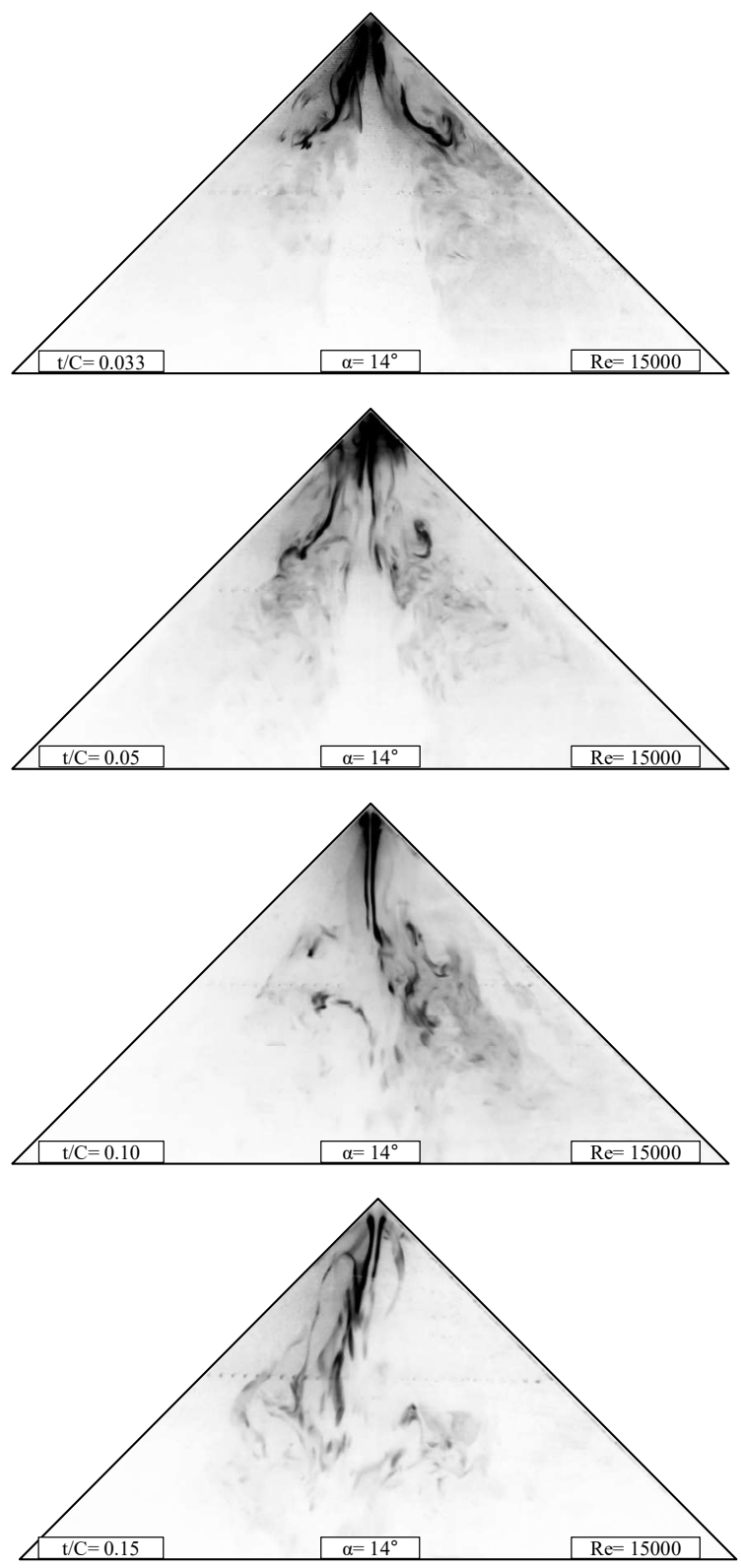

Figure D.4 Laser-illuminated surface flow smoke visualizations of $t / C=$ $0.033,0.05,0.10$ and 0.15 for $R e=1.5 \times 10^{4}$ at angle of attack of $\alpha=14 \mathrm{deg}$. 

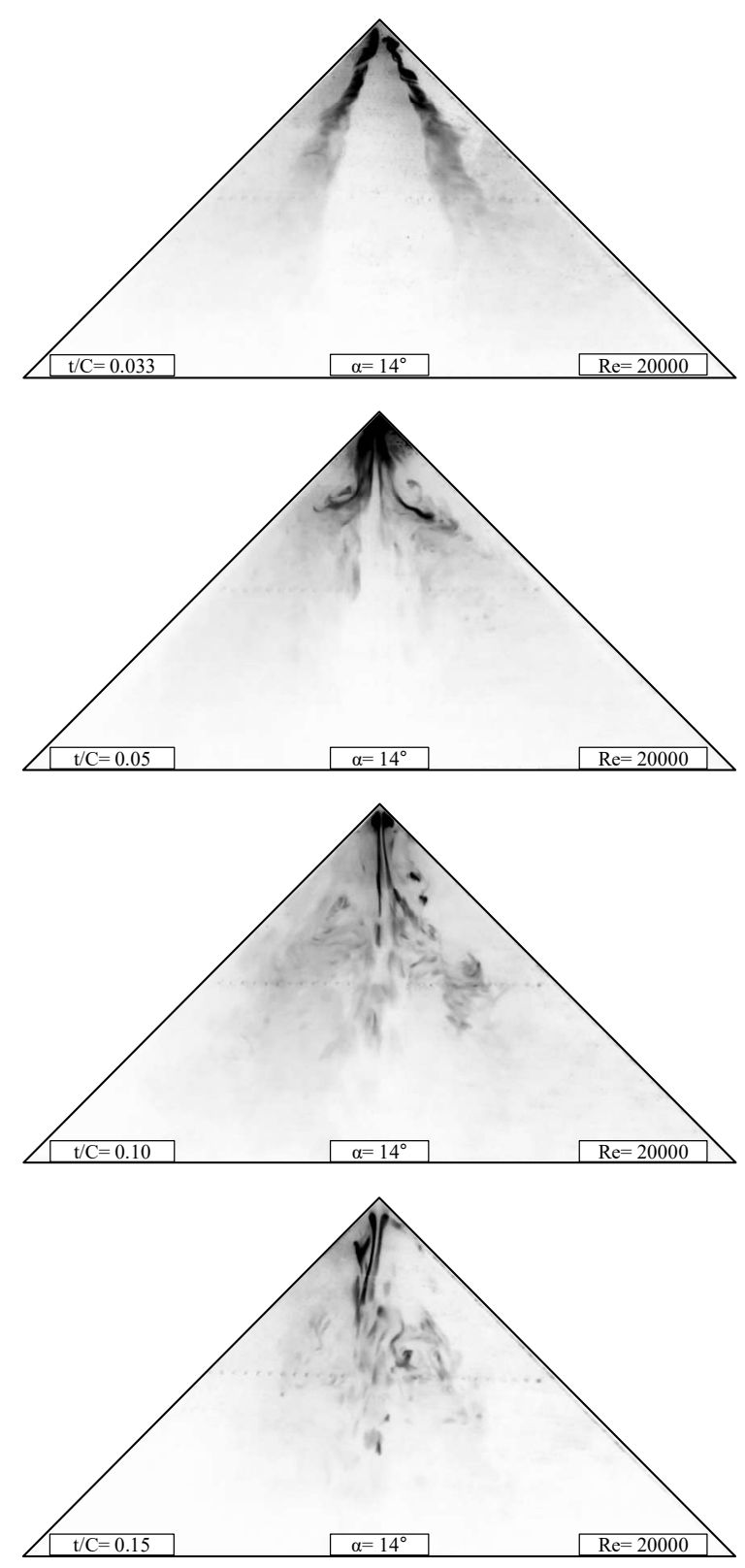

Figure D.5 Laser-illuminated surface flow smoke visualizations of $t / C=$ $0.033,0.05,0.10$ and 0.15 for $R e=2 \times 10^{4}$ at angle of attack of $\alpha=14 \mathrm{deg}$. 
F. THE DIMENSIONLESS PRESSURE DISTRIBUTION -Cp WITH RESPECT TO DIMENSIONLESS HALF SPAN FOR THICKNESS-TOCHORD RATIOS OF 0.033, 0.05, 0.10, 0.15 FOR RE=35000, 75000, 100000, $125000 \mathrm{AT} \alpha=16^{\circ} \mathrm{AND}$ FOR RE$=35000,100000 \mathrm{AT}=18^{\circ}$
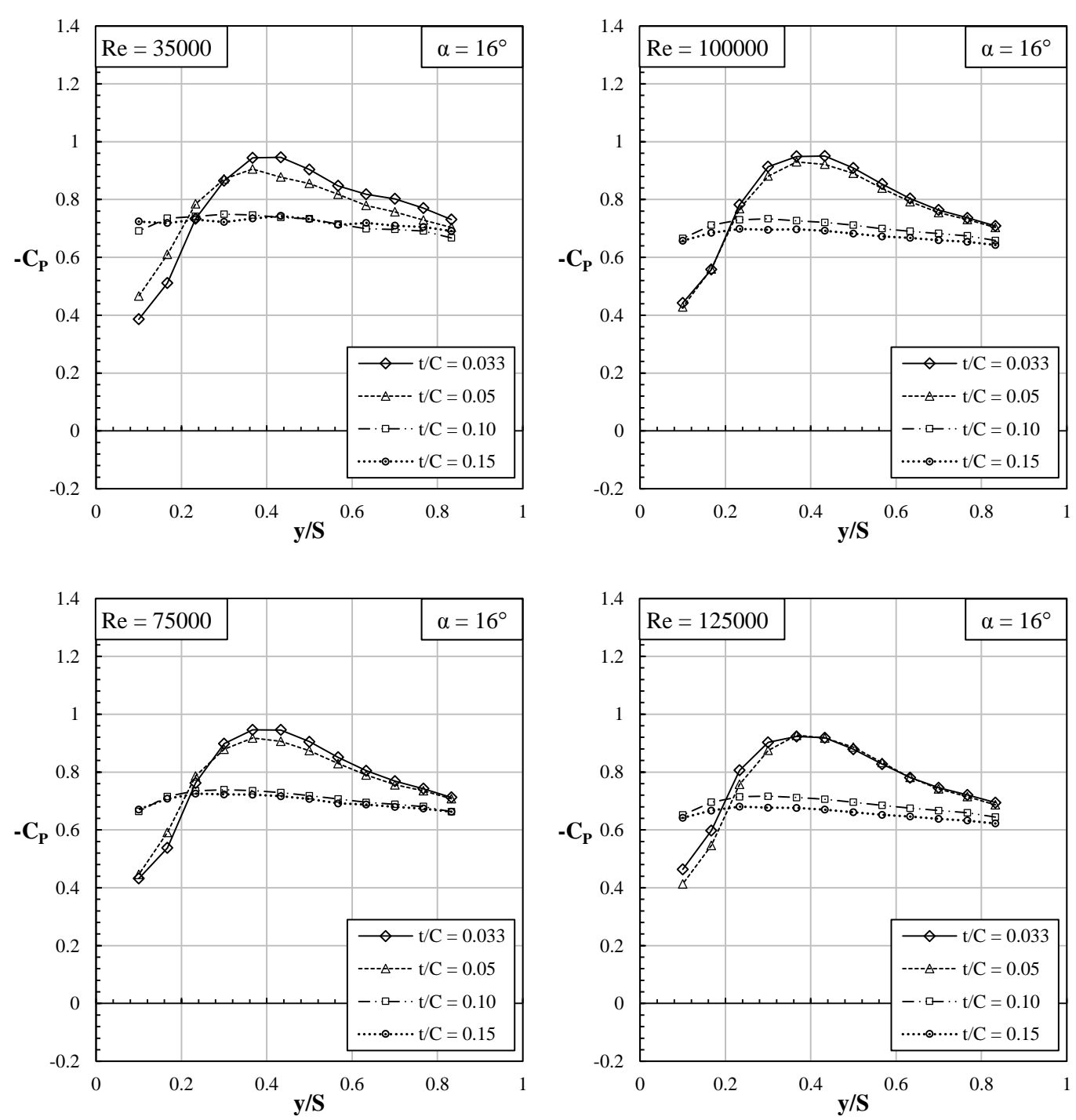

Figure F.1 The dimensionless pressure distribution $-C_{p}$ with respect to dimensionless half span for $t / C=0.033,0.05,0.10$, and 0.15 for $R e=$ $35000,75000,100000$, and 125000 at angles of attack of $\alpha=16$ degree. 

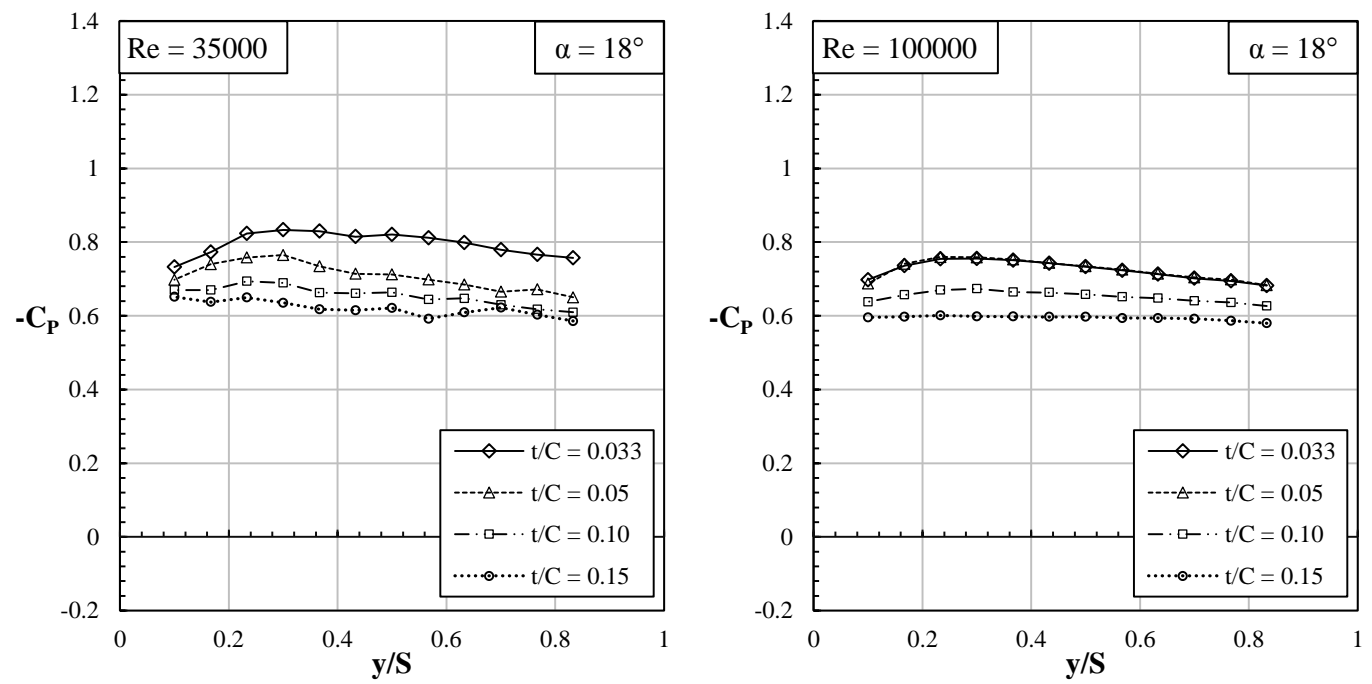

Figure F.2 The dimensionless pressure distribution $-C_{p}$ with respect to dimensionless half span for $t / C=0.033,0.05,0.10$, and 0.15 for $R e=$ 35000 and 100000 at angles of attack of $\alpha=18$ degree. 